\title{
Efficient and Selective Removal of Methoxy Protecting Groups in Carbohydrates
}

\author{
Alicia Boto, * Dácil Hernández, Rosendo Hernández, ${ }^{*}$ and Ernesto \\ Suárez
}

Instituto de Productos Naturales y Agrobiología del CSIC, Avda. Astrofisico Fco. Sanchez 3, 38206-La Laguna, Tenerife, Spain

alicia@ipna.csic.es and rhernandez@ipna.csic.es

\section{Supporting Information}

\section{Preparation of Substrates 8-13.}

The preparation of compounds $\mathbf{7 - 1 1}$ and $\mathbf{1 3}$ was carried out according to literature procedures. ${ }^{5}$ The synthesis of compound $\mathbf{1 2}$ is described below.

Benzyl 2,3,6-Tri- $O$-methyl- $\beta$-D-glucopyranoside (12). To a solution of benzyl 4- $O$-( $\alpha$-D-glucopyranosyl)$\beta$-D-glucopyranoside $\quad(\mathbf{3 3})^{10} \quad\left(\begin{array}{lllll}770 & \mathrm{mg}, & 1.78 \mathrm{mmol})\end{array}\right.$ (Scheme 5) in DMSO (10 mL) was added aqueous 50\% $\mathrm{NaOH}(1 \mathrm{~mL}, 500 \mathrm{mg}, 12.5 \mathrm{mmol})$ and $\mathrm{MeI}(1.16 \mathrm{~mL}$, $2.64 \mathrm{~g}, 18.6 \mathrm{mmol}$ ) and the mixture was stirred at room temperature overnight. The reaction mixture was poured into $\mathrm{H}_{2} \mathrm{O}$ and extracted with EtOAc. The organic layer was dried on $\mathrm{Na}_{2} \mathrm{SO}_{4}$, filtered and evaporated under vacuum. The residue was treated with $1 \mathrm{M} \mathrm{HCl}$ in $\mathrm{MeOH}$ $(35 \mathrm{~mL}, 35 \mathrm{mmol})$, and the reaction mixture was refluxed overnight; then it was poured into saturated aqueous $\mathrm{NaHCO}_{3}$ and extracted with EtOAc. The organic layer was dried on $\mathrm{Na}_{2} \mathrm{SO}_{4}$ and concentrated under vacuum. The residue was purified by chromatography on silica gel (hexanes-EtOAc, 80:20) yielding methyl 2,3,4,6-tetra- $O$ methyl- $\alpha$-D-glucopyranoside $\quad(\mathbf{3 4})^{11} \quad(129 \mathrm{mg}, 29 \%)$, methyl 2,3,4,6-tetra- $O$-methyl- $\beta$-D-glucopyranoside (35) $^{12}$ (49 mg, 11\%), and benzyl 2,3,6-tri- $O$-methyl- $\beta$-Dglucopyranoside (12) (186 mg, 33\%): Syrup; $[\alpha]_{\mathrm{D}}-20$ (c $\left.=0.2, \mathrm{CHCl}_{3}\right) ; \mathrm{IR}\left(\mathrm{CHCl}_{3}\right) v_{\max } 3597,3045,3036 \mathrm{~cm}^{-1} ;{ }^{1} \mathrm{H}$ NMR $\left(500 \mathrm{MHz}, \mathrm{CDCl}_{3}\right) \delta_{\mathrm{H}} 2.78(1 \mathrm{H}, \mathrm{br} \mathrm{s}), 3.11(2 \mathrm{H}$, $\mathrm{m}), 3.41(1 \mathrm{H}, \mathrm{ddd}, J=3.6,5.2,9.1 \mathrm{~Hz}), 3.42(3 \mathrm{H}, \mathrm{s}), 3.50$ $(1 \mathrm{H}, \mathrm{m}), 3.59(3 \mathrm{H}, \mathrm{s}), 3.63(3 \mathrm{H}, \mathrm{s}), 3.65(1 \mathrm{H}, \mathrm{dd}, J=5.2$,

\footnotetext{
${ }^{10}$ Benzyl 4- $\boldsymbol{O}$-( $\alpha$-D-Glucopyranosyl)- $\beta$-D-glucopyranoside Hirooka, M.; Koto, S. Bull. Chem. Soc. Jpn. 1998, 71, 2893-2902.

${ }^{11}$ Compound 34: Lee, C. K.; Kim, E. J.; Lee, I.-S. H. Carbohydr. Res. 1998, 309, 243-250.

${ }^{12}$ Compound 35: Cesane, P. D.; Duchaussoy, P.; Gross, B. Synthesis 1980, 953-954.
}

$10.4 \mathrm{~Hz}), 3.69(1 \mathrm{H}, \mathrm{dd}, J=3.9,10.4 \mathrm{~Hz}), 4.40(1 \mathrm{H}, \mathrm{d}, J=$ $7.6 \mathrm{~Hz}), 4.64(1 \mathrm{H}, \mathrm{d}, J=12.0 \mathrm{~Hz}), 4.93(1 \mathrm{H}, \mathrm{d}, J=12.0$ $\mathrm{Hz}), 7.28-7.37(5 \mathrm{H}, \mathrm{m}) ;{ }^{13} \mathrm{C}$ NMR $\left(125.7 \mathrm{MHz}, \mathrm{CDCl}_{3}\right)$ $\delta_{\mathrm{C}} 59.6\left(\mathrm{CH}_{3}\right), 60.3\left(\mathrm{CH}_{3}\right), 60.8\left(\mathrm{CH}_{3}\right), 71.1\left(\mathrm{CH}_{2}\right), 71.2$ $(\mathrm{CH}), 72.6\left(\mathrm{CH}_{2}\right), 73.8(\mathrm{CH}), 83.5(\mathrm{CH}), 85.7(\mathrm{CH})$, $102.5(\mathrm{CH}), 127.6(\mathrm{CH}), 127.7(2 \times \mathrm{CH}), 128.3(2 \times \mathrm{CH})$, 137.4 (C); MS m/z (rel intensity) $237\left(\mathrm{M}^{+}-\mathrm{H}-\right.$ $\mathrm{MeOCH}=\mathrm{CHOH}, 1), 91\left(\mathrm{PhCH}_{2}, 100\right)$; HRMS calcd for $\mathrm{C}_{13} \mathrm{H}_{17} \mathrm{O}_{4}$ 237.1127, found 237.1144. Anal. Calcd for $\mathrm{C}_{16} \mathrm{H}_{24} \mathrm{O}_{6}: \mathrm{C}, 61.52 ; \mathrm{H}, 7.74$. Found: $\mathrm{C}, 61.46 ; \mathrm{H}, 7.74$.

Scheme 5. Synthesis of substrate 12: methylation of compound 33 and cleavage of one glycosidic bond.

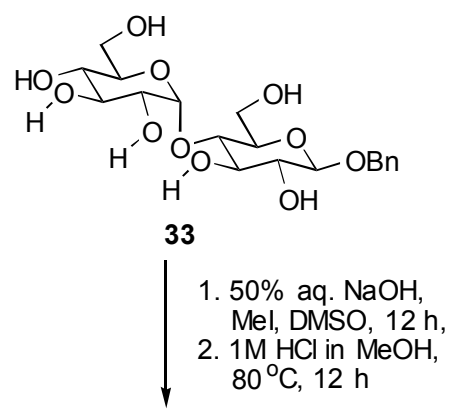

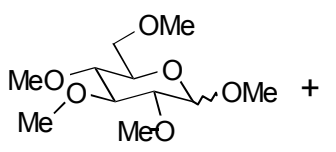

$341 \alpha-\mathrm{OMe}(29 \%)$ $351 \beta-O M e(11 \%)$

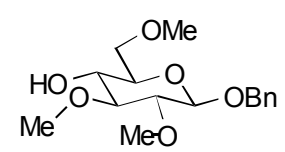

$12(33 \%)$ 
Sequential Protecting Group TransformationHydrolysis (from substrates $\mathbf{8 ,} \mathbf{9}$, and $\mathbf{1 2}$ to products $\mathbf{2 8}$, 31, and 32, respectively). General Procedure. A solution of the substrate $(0.2 \mathrm{mmol})$ in dry dichloromethane $(4$ $\mathrm{mL}$ ), placed in a pyrex round bottom flask under nitrogen, was treated with (diacetoxyiodo)benzene (DIB) (97 $\mathrm{mg}, 0.3 \mathrm{mmol}$ ) and iodine $(51 \mathrm{mg}, 0.2 \mathrm{mmol}$ ). The reaction mixture was irradiated with two tungstenfilament lamps for $1 \mathrm{~h}$ at $22-25^{\circ} \mathrm{C}$. Then the solvent was evaporated under vacuum, and glacial acetic acid $(25 \mu \mathrm{L}$, $0.5 \mathrm{mmol})$ and trifluoroacetic anhydride $(38 \mu \mathrm{L}, 0.5$ mmol) were added at $0{ }^{\circ} \mathrm{C}$ under nitrogen. The reaction mixture was allowed to reach room temperature $(1 \mathrm{~h})$ and stirred for other $2 \mathrm{~h}$. Afterwards the reagents were removed under vacuum and the residue was treated with $4 \% \mathrm{NaOH}\left(\mathrm{MeOH}-\mathrm{H}_{2} \mathrm{O}, 9: 1,0.5 \mathrm{~mL}\right)$. After stirring at room temperature for $1 \mathrm{~h}$, the reaction mixture was poured into water, neutralized with aqueous $5 \% \mathrm{HCl}$ and extracted with EtOAc. The organic layer was dried $\left(\mathrm{Na}_{2} \mathrm{SO}_{4}\right)$, evaporated under vacuum and the resulting residue was purified by chromatography on silica gel (hexanes-EtOAc).

Methyl 2,3-Di- $O$-methyl-4,6- $O$-methylene- $\alpha$-Dgalactopyranoside (14): ${ }^{1} \mathrm{H}$ NMR $\left(500 \mathrm{MHz}, \mathrm{C}_{6} \mathrm{D}_{6}\right) \delta$ $3.05(1 \mathrm{H}, \mathrm{br} \mathrm{s}), 3.13(3 \mathrm{H}, \mathrm{s}), 3.23(1 \mathrm{H}, \mathrm{dd}, J=1.8,12.1$ $\mathrm{Hz}), 3.29(3 \mathrm{H}, \mathrm{s}), 3.30(3 \mathrm{H}, \mathrm{s}), 3.54(1 \mathrm{H}, \mathrm{d}, J=3.6 \mathrm{~Hz})$, $3.59(1 \mathrm{H}, \mathrm{dd}, J=3.5,10.0 \mathrm{~Hz}), 3.89(1 \mathrm{H}, \mathrm{dd}, J=3.5$, $10.0 \mathrm{~Hz}), 3.90(1 \mathrm{H}, \mathrm{d}, J=12.2 \mathrm{~Hz}), 4.31(1 \mathrm{H}, \mathrm{d}, J=6.2$ $\mathrm{Hz}), 4.79(1 \mathrm{H}, \mathrm{d}, J=3.5 \mathrm{~Hz}), 5.07(1 \mathrm{H}, \mathrm{d}, J=6.2 \mathrm{~Hz})$; ${ }^{13} \mathrm{C}$ NMR $\left(100.6 \mathrm{MHz}, \mathrm{C}_{6} \mathrm{D}_{6}\right) \delta 55.2\left(\mathrm{CH}_{3}\right), 56.9\left(\mathrm{CH}_{3}\right)$, $58.8\left(\mathrm{CH}_{3}\right), 63.7(\mathrm{CH}), 68.9\left(\mathrm{CH}_{2}\right), 73.2(\mathrm{CH}), 77.8(\mathrm{CH})$, $77.9(\mathrm{CH}), 93.3\left(\mathrm{CH}_{2}\right), 99.4(\mathrm{CH}) . \mathrm{MS} m / z$ (rel intensity) $234\left(\mathrm{M}^{+}, 2\right), 75\left(\mathrm{C}_{3} \mathrm{H}_{6} \mathrm{O}_{2}+\mathrm{H}, 100\right)$; HRMS calcd for $\mathrm{C}_{10} \mathrm{H}_{18} \mathrm{O}_{6}$ 234.1103, found 234.1084. Anal. Calcd for $\mathrm{C}_{10} \mathrm{H}_{18} \mathrm{O}_{6}$ : C, 51.27; H, 7.75. Found: $\mathrm{C}, 51.34 ; \mathrm{H}, 7.81$.

Methyl 2,3-Di-O-methyl-4-O-(methylacetate)- $\alpha$-Dgalactopyranoside (15): Compound $\mathbf{1 5}$ could not be totally purified from its mixture with the major product 14: ${ }^{1} \mathrm{H}$ NMR $\left(500 \mathrm{MHz}, \mathrm{C}_{6} \mathrm{D}_{6}\right) \delta 1.65(3 \mathrm{H}, \mathrm{s}), 3.13(3 \mathrm{H}$, s), $3.21(3 \mathrm{H}, \mathrm{s}), 3.26(3 \mathrm{H}, \mathrm{s}), 3.51(1 \mathrm{H}, \mathrm{m}), 3.71(2 \mathrm{H}, \mathrm{m})$, $3.80(2 \mathrm{H}, \mathrm{m}), 4.03(1 \mathrm{H}, \mathrm{d}, J=2.9 \mathrm{~Hz}), 4.73(1 \mathrm{H}, \mathrm{d}, J=$ $3.5 \mathrm{~Hz}), 5.34(1 \mathrm{H}, \mathrm{d}, J=6.1 \mathrm{~Hz}), 5.36(1 \mathrm{H}, \mathrm{d}, J=6.1$ $\mathrm{Hz}) ;{ }^{13} \mathrm{C}$ NMR $\left(100.6 \mathrm{MHz}, \mathrm{C}_{6} \mathrm{D}_{6}\right) \delta 16.4\left(\mathrm{CH}_{3}\right), 55.0$ $\left(\mathrm{CH}_{3}\right), 55.2\left(\mathrm{CH}_{3}\right), 58.2\left(\mathrm{CH}_{3}\right), 61.1(\mathrm{CH}), 70.3\left(\mathrm{CH}_{2}\right)$, $75.6(\mathrm{CH}), 78.8(\mathrm{CH}), 80.0(\mathrm{CH}), 88.9\left(\mathrm{CH}_{2}\right), 98.5(\mathrm{CH})$, $167.4(\mathrm{C})$.

Methyl 2,3-Di- $O$-methyl-4,6- $O$-methylene- $\beta$-Dgalactopyranoside (16): ${ }^{1} \mathrm{H}$ NMR $\left(500 \mathrm{MHz}, \mathrm{CDCl}_{3}\right)$ $\delta 3.20(1 \mathrm{H}, \mathrm{dd}, J=3.7,9.6 \mathrm{~Hz}), 3.34(1 \mathrm{H}, \mathrm{d}, J=1.2 \mathrm{~Hz})$, $3.42(1 \mathrm{H}, \mathrm{dd}, J=7.8,9.6 \mathrm{~Hz}), 3.51(3 \mathrm{H}, \mathrm{s}), 3.55(3 \mathrm{H}, \mathrm{s})$, $3.59(3 \mathrm{H}, \mathrm{s}), 3.82(1 \mathrm{H}, \mathrm{dd}, J=1.5,12.3 \mathrm{~Hz}), 4.03(1 \mathrm{H}, \mathrm{d}$, $J=3.6 \mathrm{~Hz}), 4.18(1 \mathrm{H}, \mathrm{d}, J=12.2 \mathrm{~Hz}), 4.19(1 \mathrm{H}, \mathrm{d}, J=$ $7.7 \mathrm{~Hz}), 4.73(1 \mathrm{H}, \mathrm{d}, J=6.3 \mathrm{~Hz}), 5.19(1 \mathrm{H}, \mathrm{d}, J=6.3 \mathrm{~Hz})$; ${ }^{13} \mathrm{C}$ NMR $\left(100.6 \mathrm{MHz}, \mathrm{CDCl}_{3}\right) \delta 56.8\left(\mathrm{CH}_{3}\right), 58.1\left(\mathrm{CH}_{3}\right)$, $60.8\left(\mathrm{CH}_{3}\right), 67.0(\mathrm{CH}), 68.6\left(\mathrm{CH}_{2}\right), 72.5(\mathrm{CH}), 79.6(\mathrm{CH})$, $81.3(\mathrm{CH}), 93.5\left(\mathrm{CH}_{2}\right), 104.3(\mathrm{CH})$. MS $m / z$ (rel intensity)
$234\left(\mathrm{M}^{+}, 1\right), 75(\mathrm{MeOCH}=\mathrm{CHOH}+\mathrm{H}, 100)$; HRMS calcd for $\mathrm{C}_{10} \mathrm{H}_{18} \mathrm{O}_{6}$ 234.1103, found 234.1118. Anal. Calcd for $\mathrm{C}_{10} \mathrm{H}_{18} \mathrm{O}_{6}: \mathrm{C}, 51.27 ; \mathrm{H}, 7.75$. Found: $\mathrm{C}, 51.43$; H, 7.74 .

Methyl 2,3-Di- $O$-methyl-4- $O$-(methylacetate)- $\beta$-Dgalactopyranoside (17): IR (film) $3518,1735 \mathrm{~cm}^{-1} ;{ }^{1} \mathrm{H}$ NMR $\left(500 \mathrm{MHz}, \mathrm{CDCl}_{3}\right) \delta 2.10(3 \mathrm{H}, \mathrm{s}), 3.16(1 \mathrm{H}, \mathrm{dd}, J=$ $3.0,9.7 \mathrm{~Hz}), 3.30(1 \mathrm{H}, \mathrm{dd}, J=7.7,9.6 \mathrm{~Hz}), 3.46(1 \mathrm{H}, \mathrm{dd}$, $J=6.3,6.5 \mathrm{~Hz}), 3.53(3 \mathrm{H}, \mathrm{s}), 3.54(3 \mathrm{H}, \mathrm{s}), 3.58(3 \mathrm{H}, \mathrm{s})$, $3.68(1 \mathrm{H}, \mathrm{dd}, J=6.6,11.4 \mathrm{~Hz}), 3.78(1 \mathrm{H}, \mathrm{dd}, J=6.5$, $11.4 \mathrm{~Hz}), 4.17(1 \mathrm{H}, \mathrm{d}, J=4.1 \mathrm{~Hz}), 4.18(1 \mathrm{H}, \mathrm{d}, J=7.4$ $\mathrm{Hz}), 5.35(1 \mathrm{H}, \mathrm{d}, J=6.1 \mathrm{~Hz}), 5.38(1 \mathrm{H}, \mathrm{d}, J=6.1 \mathrm{~Hz})$; ${ }^{13} \mathrm{C}$ NMR $\left(100.6 \mathrm{MHz}, \mathrm{CDCl}_{3}\right) \delta 19.6\left(\mathrm{CH}_{3}\right), 56.9\left(\mathrm{CH}_{3}\right)$, $59.0\left(\mathrm{CH}_{3}\right), 60.4\left(\mathrm{CH}_{3}\right), 60.8\left(\mathrm{CH}_{2}\right), 73.6(2 \times \mathrm{CH}), 80.7$ $(\mathrm{CH}), 83.4(\mathrm{CH}), 89.9\left(\mathrm{CH}_{2}\right), 104.7(\mathrm{CH}), 169.9(\mathrm{C}) . \mathrm{MS}$ $m / z$ (rel intensity) $234\left(\mathrm{M}^{+}-\mathrm{MeCO}_{2} \mathrm{H}, 2\right), 75\left(\mathrm{C}_{3} \mathrm{H}_{6} \mathrm{O}_{2}+\right.$ $\mathrm{H}, 100)$; HRMS calcd for $\mathrm{C}_{10} \mathrm{H}_{18} \mathrm{O}_{6} 234.1103$, found 234.1091. Anal. Calcd for $\mathrm{C}_{12} \mathrm{H}_{22} \mathrm{O}_{8}$ : C, 48.97; H, 7.53. Found: C, 49.14; H, 7.33.

Benzyl 2,3-Di- $O$-methyl-4,6- $O$-methylene- $\alpha$-Dglucopyranoside (18). IR $\left(\mathrm{CHCl}_{3}\right) 3090,3067 \mathrm{~cm}^{-1} ;{ }^{1} \mathrm{H}$ NMR $\left(500 \mathrm{MHz}, \mathrm{CDCl}_{3}\right) \delta 3.22(1 \mathrm{H}, \mathrm{dd}, J=9.4,9.7 \mathrm{~Hz})$, $3.25(1 \mathrm{H}, \mathrm{dd}, J=3.8,9.4 \mathrm{~Hz}), 3.39(1 \mathrm{H}, \mathrm{dd}, J=10.5$, $10.5 \mathrm{~Hz}), 3.41(3 \mathrm{H}, \mathrm{s}), 3.63(3 \mathrm{H}, \mathrm{s}), 3.65(1 \mathrm{H}, \mathrm{dd}, J=9.3$, $9.4 \mathrm{~Hz}), 3.73(1 \mathrm{H}, \mathrm{ddd}, J=4.9,10.0,10.0 \mathrm{~Hz}), 4.01(1 \mathrm{H}$, dd, $J=4.9,10.2 \mathrm{~Hz}), 4.60(1 \mathrm{H}, \mathrm{d}, J=6.3 \mathrm{~Hz}), 4.62(1 \mathrm{H}$, d, $J=12.2 \mathrm{~Hz}), 4.73(1 \mathrm{H}, \mathrm{d}, J=12.2 \mathrm{~Hz}), 4.98(1 \mathrm{H}, \mathrm{d}, J$ $=3.8 \mathrm{~Hz}), 5.06(1 \mathrm{H}, \mathrm{d}, J=6.3 \mathrm{~Hz}), 7.31(1 \mathrm{H}, \mathrm{dd}, J=7.1$, $7.1 \mathrm{~Hz}), 7.37(2 \mathrm{H}, \mathrm{dd}, J=7.1,7.6 \mathrm{~Hz}), 7.39(2 \mathrm{H}, \mathrm{d}, J=$ $7.0 \mathrm{~Hz}) ;{ }^{13} \mathrm{C}$ NMR $\left(125.7 \mathrm{MHz}, \mathrm{CDCl}_{3}\right) \delta 58.6\left(\mathrm{CH}_{3}\right)$, $61.0\left(\mathrm{CH}_{3}\right), 62.7(\mathrm{CH}), 68.7\left(\mathrm{CH}_{2}\right), 69.5\left(\mathrm{CH}_{2}\right), 79.5$ $(\mathrm{CH}), 81.6(\mathrm{CH}), 81.7(\mathrm{CH}), 93.7\left(\mathrm{CH}_{2}\right), 95.8(\mathrm{CH})$, $128.0(\mathrm{CH}), 128.3(2 \times \mathrm{CH}), 128.4(2 \times \mathrm{CH}), 136.9(\mathrm{C})$. MS $m / z$ (rel intensity) $219\left(\mathrm{M}^{+}-\mathrm{PhCH}_{2}, 2\right), 91\left(\mathrm{PhCH}_{2}\right.$, 100); HRMS calcd for $\mathrm{C}_{9} \mathrm{H}_{15} \mathrm{O}_{6}$ 219.0869, found 219.0938. Anal. Calcd for $\mathrm{C}_{16} \mathrm{H}_{22} \mathrm{O}_{6}$ : C, 61.92; H, 7.15. Found: C, 61.87; H, 7.11.

Benzyl 2,3-Di- $O$-methyl-4,6- $O$-methylene- $\beta$-Dglucopyranoside (19): IR $\left(\mathrm{CHCl}_{3}\right) .3090,3063 \mathrm{~cm}^{-1} ;{ }^{1} \mathrm{H}$ NMR (500 MHz, $\left.\mathrm{CDCl}_{3}\right) \delta 3.12(1 \mathrm{H}, \mathrm{dd}, J=7.8,8.1 \mathrm{~Hz})$, $3.23-3.30(3 \mathrm{H}, \mathrm{m}), 3.48(1 \mathrm{H}, \mathrm{dd}, J=9.8,10.2 \mathrm{~Hz}), 3.60$ $(3 \mathrm{H}, \mathrm{s}), 3.61(3 \mathrm{H}, \mathrm{s}), 4.20(1 \mathrm{H}, \mathrm{dd}, J=4.3,10.6 \mathrm{~Hz}), 4.48$ $(1 \mathrm{H}, \mathrm{d}, J=7.6 \mathrm{~Hz}), 4.60(1 \mathrm{H}, \mathrm{d}, J=6.3 \mathrm{~Hz}), 4.64(1 \mathrm{H}, \mathrm{d}$, $J=12.0 \mathrm{~Hz}), 4.89(1 \mathrm{H}, \mathrm{d}, J=11.8 \mathrm{~Hz}), 5.05(1 \mathrm{H}, \mathrm{d}, J=$ $6.3 \mathrm{~Hz}), 7.28-7.36(5 \mathrm{H}, \mathrm{m}) ;{ }^{13} \mathrm{C}$ NMR $(125.7 \mathrm{MHz}$, $\left.\mathrm{CDCl}_{3}\right) \delta 60.8\left(\mathrm{CH}_{3}\right), 60.9\left(\mathrm{CH}_{3}\right), 66.2(\mathrm{CH}), 68.5\left(\mathrm{CH}_{2}\right)$, $71.3\left(\mathrm{CH}_{2}\right), 80.7(\mathrm{CH}), 82.5(\mathrm{CH}), 84.2(\mathrm{CH}), 93.6\left(\mathrm{CH}_{2}\right)$, $102.9(\mathrm{CH}), 127.8(2 \times \mathrm{CH}), 127.9(\mathrm{CH}), 128.4(2 \times \mathrm{CH})$, 137.1 (C). MS $m / z$ (rel intensity) $219\left(\mathrm{M}^{+}-\mathrm{PhCH}_{2},<1\right.$ ), $91\left(\mathrm{PhCH}_{2}, 100\right)$; HRMS calcd for $\mathrm{C}_{9} \mathrm{H}_{15} \mathrm{O}_{6}, 219.0869$, found 219.0891. Anal. Calcd for $\mathrm{C}_{16} \mathrm{H}_{22} \mathrm{O}_{6}: \mathrm{C}, 61.92 ; \mathrm{H}$, 7.15. Found: C, 62.17; H, 6.82 .

Methyl 2,6-Di- $O$-methyl-3- $O$-(methylacetate)- $\beta$-Dglucopyranoside (20): IR (film) 3518, $1742 \mathrm{~cm}^{-1} ;{ }^{1} \mathrm{H}$ NMR $\left(500 \mathrm{MHz}, \mathrm{CDCl}_{3}\right) \delta 2.10(3 \mathrm{H}, \mathrm{s}), 3.01(1 \mathrm{H}, \mathrm{dd}, J$ 
$=8.2,8.3 \mathrm{~Hz}), 3.41(1 \mathrm{H}, \mathrm{m}), 3.42(3 \mathrm{H}, \mathrm{s}), 3.53(3 \mathrm{H}, \mathrm{s})$, $3.54(3 \mathrm{H}, \mathrm{s}), 3.55(1 \mathrm{H}, \mathrm{m}), 3.64(1 \mathrm{H}, \mathrm{dd}, J=5.1,10.3$ $\mathrm{Hz}), 3.68(1 \mathrm{H}, \mathrm{dd}, J=3.8,10.4 \mathrm{~Hz}), 4.19(1 \mathrm{H}, \mathrm{d}, J=7.8$ $\mathrm{Hz}), 5.38(1 \mathrm{H}, \mathrm{d}, J=6.0 \mathrm{~Hz}), 5.46(1 \mathrm{H}, \mathrm{d}, J=6.0 \mathrm{~Hz})$; ${ }^{13} \mathrm{C} \mathrm{NMR}\left(125.7 \mathrm{MHz}, \mathrm{CDCl}_{3}\right) \delta 21.1\left(\mathrm{CH}_{3}\right), 57.0\left(\mathrm{CH}_{3}\right)$, $59.6\left(\mathrm{CH}_{3}\right), 60.6\left(\mathrm{CH}_{3}\right), 70.5(\mathrm{CH}), 72.6\left(\mathrm{CH}_{2}\right), 73.8$ $(\mathrm{CH}), 82.5(\mathrm{CH}), 84.3(\mathrm{CH}), 89.9\left(\mathrm{CH}_{2}\right), 104.4(\mathrm{CH})$, 170.7 (C). MS $m / z$ (rel intensity) $234\left(\mathrm{M}^{+}-\mathrm{MeCO}_{2} \mathrm{H}\right.$, $<1), 74\left(\mathrm{C}_{3} \mathrm{H}_{6} \mathrm{O}_{2}, 100\right)$; HRMS calcd for $\mathrm{C}_{10} \mathrm{H}_{18} \mathrm{O}_{6}$, 234.1103, found 234.1053. Anal. Calcd for $\mathrm{C}_{12} \mathrm{H}_{22} \mathrm{O}_{8}$ : C, 48.97; H, 7.53. Found: C, 49.29; H, 7.84.

Benzyl 2,6-Di- $O$-methyl-3- $O$-(methylacetate)- $\beta$-Dglucopyranoside (22): IR (film) $3592,1739 \mathrm{~cm}^{-1} ;{ }^{1} \mathrm{H}$ NMR $\left(500 \mathrm{MHz}, \mathrm{CDCl}_{3}\right) \delta 2.10(3 \mathrm{H}, \mathrm{s}), 3.11(1 \mathrm{H}, \mathrm{dd}, J=$ $8.0,8.8 \mathrm{~Hz}), 3.40(1 \mathrm{H}, \mathrm{m}), 3.42(3 \mathrm{H}, \mathrm{s}), 3.50(1 \mathrm{H}, \mathrm{dd}, J=$ 8.8, 9.2 Hz), $3.54(1 \mathrm{H}, \mathrm{dd}, J=7.5,9.0 \mathrm{~Hz}), 3.56(3 \mathrm{H}, \mathrm{s})$, $3.65(1 \mathrm{H}, \mathrm{dd}, J=5.1,10.5 \mathrm{~Hz}), 3.70(1 \mathrm{H}, \mathrm{dd}, J=3.7$, $10.4 \mathrm{~Hz}), 4.40(1 \mathrm{H}, \mathrm{d}, J=7.8 \mathrm{~Hz}), 4.63(1 \mathrm{H}, \mathrm{d}, J=11.9$ $\mathrm{Hz}), 4.93(1 \mathrm{H}, \mathrm{d}, J=11.9 \mathrm{~Hz}), 5.38(1 \mathrm{H}, \mathrm{d}, J=6.1 \mathrm{~Hz})$, $5.45(1 \mathrm{H}, \mathrm{d}, J=6.0 \mathrm{~Hz}), 7.35-7.42(5 \mathrm{H}, \mathrm{m}) ;{ }^{13} \mathrm{C} \mathrm{NMR}$ $\left(100.6 \mathrm{MHz}, \mathrm{CDCl}_{3}\right) \delta 30.0\left(\mathrm{CH}_{3}\right), 60.0\left(\mathrm{CH}_{3}\right), 61.1$ $\left(\mathrm{CH}_{3}\right), 70.8(\mathrm{CH}), 71.4\left(\mathrm{CH}_{2}\right), 73.8\left(\mathrm{CH}_{2}\right), 74.3(\mathrm{CH})$, $83.0(\mathrm{CH}), 84.7(\mathrm{CH}), 90.3\left(\mathrm{CH}_{2}\right), 102.8(\mathrm{CH}), 128.1(2 \times$ $\mathrm{CH}), 128.2(\mathrm{CH}), 128.8(2 \times \mathrm{CH}), 137.7$ (C) $164.8(\mathrm{C})$. MS $m / z$ (rel intensity) $295\left(\mathrm{M}^{+}-\mathrm{H}-\mathrm{MeOCH}_{2} \mathrm{CHO},<1\right)$, $91\left(\mathrm{PhCH}_{2}, 100\right)$; HRMS calcd for $\mathrm{C}_{15} \mathrm{H}_{19} \mathrm{O}_{6}, 295.1182$, found 219.1191. Anal. Calcd for $\mathrm{C}_{18} \mathrm{H}_{26} \mathrm{O}_{8}$ : C, 58.37; H, 7.08. Found: C, 58.07; H, 7.43.

Benzyl 2,6-Di- $\boldsymbol{O}$-methyl-3,4- $\boldsymbol{O}$-methylene- $\boldsymbol{\beta}$-Dglucopyranoside (23): IR (neat) $3095,3067 \mathrm{~cm}^{-1} ;{ }^{1} \mathrm{H}$ NMR (500 MHz, $\left.\mathrm{CDCl}_{3}\right) \delta 3.49(1 \mathrm{H}, \mathrm{m}), 3.43(3 \mathrm{H}, \mathrm{s})$, $3.51(1 \mathrm{H}, \mathrm{m}), 3.53(1 \mathrm{H}, \mathrm{m}), 3.61(3 \mathrm{H}, \mathrm{s}), 3.65(1 \mathrm{H}, \mathrm{dd}, J$ $=3.8,8.4 \mathrm{~Hz}), 4.13(1 \mathrm{H}, \mathrm{m}), 4.35(1 \mathrm{H}, \mathrm{dd}, J=4.5,4.5$ $\mathrm{Hz}), 4.67(1 \mathrm{H}, \mathrm{d}, J=11.9 \mathrm{~Hz}), 4.81(1 \mathrm{H}, \mathrm{d}, J=7.6 \mathrm{~Hz})$, $4.94(1 \mathrm{H}, \mathrm{d}, J=11.9 \mathrm{~Hz}), 4.97(1 \mathrm{H}, \mathrm{s}), 5.23(1 \mathrm{H}, \mathrm{s})$, 7.27-7.38 (5H, m); $\left.{ }^{13} \mathrm{C} \mathrm{NMR} \mathrm{(125.7} \mathrm{MHz,} \mathrm{CDCl}_{3}\right) \delta 59.4$ $\left(\mathrm{CH}_{3}\right), 59.6\left(\mathrm{CH}_{3}\right), 70.7\left(\mathrm{CH}_{2}\right), 71.7(\mathrm{CH}), 71.9\left(\mathrm{CH}_{2}\right)$, $73.1(\mathrm{CH}), 75.8(\mathrm{CH}), 77.7(\mathrm{CH}), 95.7\left(\mathrm{CH}_{2}\right), 99.8(\mathrm{CH})$, $127.6(\mathrm{CH}), 127.7(2 \times \mathrm{CH}), 128.3(2 \times \mathrm{CH}), 137.3(\mathrm{C})$. MS $m / z$ (rel intensity) $267\left(\mathrm{M}^{+}-\mathrm{H}-\mathrm{CH}_{2}=\mathrm{CO}, 3\right), 91$ $\left(\mathrm{PhCH}_{2}, 100\right)$; HRMS calcd for $\mathrm{C}_{14} \mathrm{H}_{19} \mathrm{O}_{5}, 267.1232$, found 267.1227. Anal. Calcd for $\mathrm{C}_{16} \mathrm{H}_{22} \mathrm{O}_{6}$ : C, 61.92; $\mathrm{H}$, 7.15. Found: C, 62.27; H, 7.46.

\footnotetext{
Methyl 4-O-Methyl-3- $\boldsymbol{O}$-(methylacetate)- $\boldsymbol{\beta}$-Lrhamnopyranoside (24): IR (film) $3580,1740 \mathrm{~cm}^{-1} ;{ }^{1} \mathrm{H}$ NMR $\left(500 \mathrm{MHz}, \mathrm{CDCl}_{3}\right) \delta 1.31(3 \mathrm{H}, \mathrm{d}, J=6.3 \mathrm{~Hz})$, $2.10(3 \mathrm{H}, \mathrm{s}), 3.12(1 \mathrm{H}, \mathrm{dd}, J=9.4,9.4 \mathrm{~Hz}), 3.35(3 \mathrm{H}, \mathrm{s})$, $3.50(3 \mathrm{H}, \mathrm{s}), 3.60(1 \mathrm{H}$, dddd, $J=6.2,6.3,6.4,9.6 \mathrm{~Hz})$, $3.82(1 \mathrm{H}, \mathrm{dd}, J=3.4,9.3 \mathrm{~Hz}), 3.95(1 \mathrm{H}, \mathrm{dd}, J=1.6,3.3$ $\mathrm{Hz}), 4.65(1 \mathrm{H}$, br s), $5.31(1 \mathrm{H}, \mathrm{d}, J=6.3 \mathrm{~Hz}), 5.46(1 \mathrm{H}$, $\mathrm{d}, J=6.2 \mathrm{~Hz}) ;{ }^{13} \mathrm{C} \mathrm{NMR}\left(100.6 \mathrm{MHz}, \mathrm{CDCl}_{3}\right) \delta 17.7$ $\left(\mathrm{CH}_{3}\right), 21.1\left(\mathrm{CH}_{3}\right), 54.8\left(\mathrm{CH}_{3}\right), 61.0\left(\mathrm{CH}_{3}\right), 67.3(\mathrm{CH})$, $70.1(\mathrm{CH}), 80.6(\mathrm{CH}), 81.4(\mathrm{CH}), 88.7\left(\mathrm{CH}_{2}\right), 100.0$ (CH), 170.4 (C). MS m/z (rel intensity) $204\left(\mathrm{M}^{+}-\right.$ $\left.\mathrm{MeCO}_{2} \mathrm{H}, 1\right), 72$ (100); HRMS calcd for $\mathrm{C}_{9} \mathrm{H}_{16} \mathrm{O}_{5}$,
}

204.0998, found 204.0963. Anal. Calcd for $\mathrm{C}_{11} \mathrm{H}_{20} \mathrm{O}_{7}$ : C, 49.99; H, 7.63. Found: C, 49.67; H, 7.98.

Methyl 4-O-Methyl-2,3- $O$-methylene- $\beta$-Lrhamnopyranoside (25): Compound 25 could not be totally purified from its mixture with the major product 24: ${ }^{1} \mathrm{H}$ NMR $\left(500 \mathrm{MHz}, \mathrm{CDCl}_{3}\right) \delta 1.26(3 \mathrm{H}, \mathrm{d}, J=6.3$ $\mathrm{Hz}), 2.75(1 \mathrm{H}, \mathrm{dd}, J=9.3,9.3 \mathrm{~Hz}), 3.40(3 \mathrm{H}, \mathrm{s}), 3.51$ $(3 \mathrm{H}, \mathrm{s}), 3.58(2 \mathrm{H}, \mathrm{m}), 3.72(1 \mathrm{H}, \mathrm{dd}, J=9.3,9.2 \mathrm{~Hz}), 4.67$ $(1 \mathrm{H}, \mathrm{d}, J=3.9 \mathrm{~Hz}), 5.40(1 \mathrm{H}, \mathrm{d}, J=6.3 \mathrm{~Hz}), 5.49(1 \mathrm{H}, \mathrm{d}$, $J=6.3 \mathrm{~Hz}) ;{ }^{13} \mathrm{C} \mathrm{NMR}\left(100.6 \mathrm{MHz}, \mathrm{CDCl}_{3}\right) \delta 17.6\left(\mathrm{CH}_{3}\right)$, $55.1\left(\mathrm{CH}_{3}\right), 60.9\left(\mathrm{CH}_{3}\right), 66.7(\mathrm{CH}), 72.1(\mathrm{CH}), 82.9(\mathrm{CH})$, $84.7(\mathrm{CH}), 89.6\left(\mathrm{CH}_{2}\right), 99.0(\mathrm{CH})$.

$\begin{array}{ccr}\text { Methyl 6-O-Acetyl-2,3-di- } O \text {-methyl-4- } O \text { - } & \text { - } \\ \text { (methylacetate)- } \beta \text {-D-galactopyranoside } & (26): & \text { IR }\end{array}$
$\left(\mathrm{CHCl}_{3}\right) 1740 \mathrm{~cm}^{-1} ;{ }^{1} \mathrm{H}$ NMR $\left(500 \mathrm{MHz}, \mathrm{CDCl}_{3}\right) \delta 2.08$ $(3 \mathrm{H}, \mathrm{s}), 2.09(3 \mathrm{H}, \mathrm{s}), 3.13(1 \mathrm{H}, \mathrm{dd}, J=3.0,9.6 \mathrm{~Hz}), 3.30$ $(1 \mathrm{H}, \mathrm{dd}, J=7.6,9.6 \mathrm{~Hz}), 3.52(3 \mathrm{H}, \mathrm{s}), 3.54(3 \mathrm{H}, \mathrm{s}), 3.56$ $(1 \mathrm{H}, \mathrm{dd}, J=6.3,6.6 \mathrm{~Hz}), 3.57(3 \mathrm{H}, \mathrm{s}), 4.11(1 \mathrm{H}, \mathrm{d}, J=$ $2.9 \mathrm{~Hz}), 4.15(1 \mathrm{H}, \mathrm{d}, J=7.6 \mathrm{~Hz}), 4.17(1 \mathrm{H}, \mathrm{dd}, J=6.3$, $11.2 \mathrm{~Hz}), 4.25(1 \mathrm{H}, \mathrm{dd}, J=6.4,11.2 \mathrm{~Hz}), 5.34(1 \mathrm{H}, \mathrm{d}, J=$ $6.4 \mathrm{~Hz}), 5.42(1 \mathrm{H}, \mathrm{d}, J=6.4 \mathrm{~Hz}) ;{ }^{13} \mathrm{C} \mathrm{NMR}(125.7 \mathrm{MHz}$, $\left.\mathrm{CDCl}_{3}\right) \delta 20.8\left(\mathrm{CH}_{3}\right), 21.0\left(\mathrm{CH}_{3}\right), 56.8\left(\mathrm{CH}_{3}\right), 59.0$ $\left(\mathrm{CH}_{3}\right), 60.8\left(\mathrm{CH}_{3}\right), 62.7\left(\mathrm{CH}_{2}\right), 71.1(\mathrm{CH}), 73.2(\mathrm{CH})$, $80.4(\mathrm{CH}), 83.2(\mathrm{CH}), 89.0\left(\mathrm{CH}_{2}\right), 104.5(\mathrm{CH}), 170.6(\mathrm{C})$, 170.8 (C). MS $m / z$ (rel intensity) $277\left(\mathrm{M}^{+}-\mathrm{MeCO}_{2}, 1\right)$, $88(\mathrm{MeOCH}=\mathrm{CHOMe}, 100)$; HRMS calcd for $\mathrm{C}_{12} \mathrm{H}_{21} \mathrm{O}_{7}$, 277.1287, found 277.1236. Anal. Calcd for $\mathrm{C}_{14} \mathrm{H}_{24} \mathrm{O}_{9}$ : C, 50.00; H, 7.19. Found: C, 50.09; H, 7.22.

Methyl 6- $O$-Acetyl-2,3-di- $O$-methyl- $\beta$-Dgalactopyranoside (27): IR (film) $3570,1740 \mathrm{~cm}^{-1} ;{ }^{1} \mathrm{H}$ $\operatorname{NMR}\left(500 \mathrm{MHz}, \mathrm{CDCl}_{3}\right) \delta 2.09(3 \mathrm{H}, \mathrm{s}), 3.17(1 \mathrm{H}, \mathrm{dd}, J=$ 3.2, $9.3 \mathrm{~Hz}), 3.24(1 \mathrm{H}, \mathrm{dd}, J=7.7,9.2 \mathrm{~Hz}), 3.51(3 \mathrm{H}, \mathrm{s})$, $3.54(3 \mathrm{H}, \mathrm{s}), 3.57(3 \mathrm{H}, \mathrm{s}), 3.59(1 \mathrm{H}, \mathrm{dd}, J=5.8,6.8 \mathrm{~Hz})$, $3.99(1 \mathrm{H}, \mathrm{d}, J=3.2 \mathrm{~Hz}), 4.16(1 \mathrm{H}, \mathrm{d}, J=7.5 \mathrm{~Hz}), 4.34$ $(2 \mathrm{H}, \mathrm{d}, J=6.2 \mathrm{~Hz}) ;{ }^{13} \mathrm{C} \mathrm{NMR}\left(125.7 \mathrm{MHz}, \mathrm{CDCl}_{3}\right) \delta_{\mathrm{C}}$ $20.9\left(\mathrm{CH}_{3}\right), 56.8\left(\mathrm{CH}_{3}\right), 57.9\left(\mathrm{CH}_{3}\right), 60.8\left(\mathrm{CH}_{3}\right), 63.0$ $\left(\mathrm{CH}_{2}\right), 65.5(\mathrm{CH}), 71.7(\mathrm{CH}), 80.1(\mathrm{CH}), 82.5(\mathrm{CH})$, $104.3(\mathrm{CH}), 170.8(\mathrm{C})$. MS $\mathrm{m} / z$ (rel intensity) $247\left(\mathrm{M}^{+}-\right.$ $\mathrm{OH},<1), 88$ (MeOCH=CHOMe, 100); HRMS calcd for $\mathrm{C}_{11} \mathrm{H}_{19} \mathrm{O}_{6}, 247.1182$, found 247.1196. Anal. Calcd for $\mathrm{C}_{11} \mathrm{H}_{20} \mathrm{O}_{7}$ : C, 49.99; H, 7.63. Found: C, 50.13; H, 7.52.

\section{Benzyl 6-O-Acetyl-2,3-di-O-methyl-4- $O$ -} (methylcetate)- $\alpha$-D-glucopyranoside (29): IR $\left(\mathrm{CHCl}_{3}\right)$ 3091, 3067, $1740 \mathrm{~cm}^{-1} ;{ }^{1} \mathrm{H}$ NMR $\left(500 \mathrm{Mz}, \mathrm{CDCl}_{3}\right) \delta 2.08$ $(3 \mathrm{H}, \mathrm{s}), 2.10(3 \mathrm{H}, \mathrm{s}), 3.21(1 \mathrm{H}, \mathrm{dd}, J=3.7,9.2 \mathrm{~Hz}), 3.41$ $(3 \mathrm{H}, \mathrm{s}), 3.55(1 \mathrm{H}, \mathrm{dd}, J=8.5,8.8 \mathrm{~Hz}), 3.58(1 \mathrm{H}, \mathrm{dd}, J=$ 8.0, $8.5 \mathrm{~Hz}), 3.60(3 \mathrm{H}, \mathrm{s}), 3.78(1 \mathrm{H}, \mathrm{ddd}, J=3.5,3.7,9.4$ $\mathrm{Hz}), 4.15(1 \mathrm{H}, \mathrm{dd}, J=3.8,12.4 \mathrm{~Hz}), 4.17(1 \mathrm{H}, \mathrm{dd}, J=$ $4.0,12.5 \mathrm{~Hz}), 4.59(1 \mathrm{H}, \mathrm{d}, J=12.1 \mathrm{~Hz}), 4.70(1 \mathrm{H}, \mathrm{d}, J=$ $12.2 \mathrm{~Hz}), 4.98(1 \mathrm{H}, \mathrm{d}, J=3.7 \mathrm{~Hz}), 5.33(1 \mathrm{H}, \mathrm{d}, J=6.3$ $\mathrm{Hz}), 5.42(1 \mathrm{H}, \mathrm{d}, J=6.3 \mathrm{~Hz}), 7.30(1 \mathrm{H}, \mathrm{dd}, J=6.9,7.0$ $\mathrm{Hz}), 7.34$ (2H, dd, $J=7.0,7.6 \mathrm{~Hz}), 7.37(2 \mathrm{H}, \mathrm{d}, J=7.0$ $\mathrm{Hz}) ;{ }^{13} \mathrm{C}$ NMR $\left(125.7 \mathrm{MHz}, \mathrm{CDCl}_{3}\right) \delta 20.8\left(\mathrm{CH}_{3}\right), 20.9$ $\left(\mathrm{CH}_{3}\right), 58.4\left(\mathrm{CH}_{3}\right), 61.2\left(\mathrm{CH}_{3}\right), 62.8\left(\mathrm{CH}_{2}\right), 68.1(\mathrm{CH})$, $69.2\left(\mathrm{CH}_{2}\right), 77.5(\mathrm{CH}), 81.7(\mathrm{CH}), 82.5(\mathrm{CH}), 89.2\left(\mathrm{CH}_{2}\right)$, 
$94.9(\mathrm{CH}), 128.0(\mathrm{CH}), 128.3(2 \times \mathrm{CH}), 128.4(2 \times \mathrm{CH})$, 136.8 (C), 170.5 (C), 170.8 (C). MS $\mathrm{m} / z$ (rel intensity) $353\left(\mathrm{M}^{+}-\mathrm{MeCO}_{2},<1\right), 91\left(\mathrm{PhCH}_{2}, 100\right)$; HRMS calcd for $\mathrm{C}_{18} \mathrm{H}_{25} \mathrm{O}_{7} 353.1600$, found 353.1588. Anal. Calcd for $\mathrm{C}_{20} \mathrm{H}_{28} \mathrm{O}_{9}$ : C, 58.24; H, 6.84. Found: C, 58.19; H, 6.99.

\section{Benzyl 6-O-Acetyl-2,3-di- $O$-methyl- $\alpha$-D-} glucopyranoside (30): $\mathrm{IR}\left(\mathrm{CHCl}_{3}\right)$ 3595, 3090, 3066, $1736 \mathrm{~cm}^{-1} ;{ }^{1} \mathrm{H}$ NMR $\left(500 \mathrm{Mz}, \mathrm{CDCl}_{3}\right) \delta 2.11(3 \mathrm{H}, \mathrm{s})$, $3.22(1 \mathrm{H}, \mathrm{dd}, J=3.7,9.5 \mathrm{~Hz}), 3.38(1 \mathrm{H}, \mathrm{dd}, J=9.3,9.6$ $\mathrm{Hz}), 3.39(3 \mathrm{H}, \mathrm{s}), 3.52(1 \mathrm{H}, \mathrm{dd}, J=9.2,9.2 \mathrm{~Hz}), 3.65$ $(3 \mathrm{H}, \mathrm{s}), 3.78(1 \mathrm{H}, \mathrm{ddd}, J=2.3,4.5,10.9 \mathrm{~Hz}), 4.14(1 \mathrm{H}$, $\mathrm{dd}, J=2.2,12.2 \mathrm{~Hz}), 4.41(1 \mathrm{H}, \mathrm{dd}, J=4.5,12.1 \mathrm{~Hz})$, $4.62(1 \mathrm{H}, \mathrm{d}, J=12.2 \mathrm{~Hz}), 4.73(1 \mathrm{H}, \mathrm{d}, J=12.2 \mathrm{~Hz}), 5.01$ $(1 \mathrm{H}, \mathrm{d}, J=3.7 \mathrm{~Hz}), 7.30(1 \mathrm{H}, \mathrm{d}, J=7.1,7.1 \mathrm{~Hz}), 7.35$ $(2 \mathrm{H}, \mathrm{dd}, J=7.0,7.6 \mathrm{~Hz}), 7.40(2 \mathrm{H}, \mathrm{d}, J=7.0 \mathrm{~Hz}) ;{ }^{13} \mathrm{C}$ NMR $\left(125.7 \mathrm{MHz}, \mathrm{CDCl}_{3}\right) \delta 20.8\left(\mathrm{CH}_{3}\right), 58.1\left(\mathrm{CH}_{3}\right)$, $61.3\left(\mathrm{CH}_{3}\right), 63.2\left(\mathrm{CH}_{2}\right), 69.5\left(\mathrm{CH}_{2}\right), 69.6(\mathrm{CH}), 70.0$ $(\mathrm{CH}), 81.6(\mathrm{CH}), 82.3(\mathrm{CH}), 95.1(\mathrm{CH}), 128.0(\mathrm{CH})$, $128.3(2 \times \mathrm{CH}), 128.4(2 \times \mathrm{CH}), 136.9(\mathrm{C}), 171.4(\mathrm{C})$. MS $m / z$ (rel intensity) $341\left(\mathrm{M}^{+}+\mathrm{H},<1\right), 91\left(\mathrm{PhCH}_{2}\right.$, 100); HRMS calcd for $\mathrm{C}_{17} \mathrm{H}_{25} \mathrm{O}_{7} 341.1600$, found 341.1568. Anal. Calcd for $\mathrm{C}_{17} \mathrm{H}_{24} \mathrm{O}_{7}$ : C, 59.99; H, 7.11. Found: C, 59.75; H, 7.37.

Benzyl 2,3-Di- $O$-methyl- $\alpha$-D-glucopyranoside (31): IR (film) 3593, 3090, $3067 \mathrm{~cm}^{-1}$; ${ }^{1} \mathrm{H}$ NMR $(500 \mathrm{MHz}$, $\left.\mathrm{CDCl}_{3}\right) \delta 3.21(1 \mathrm{H}, \mathrm{dd}, J=3.5,9.3 \mathrm{~Hz}), 3.39(3 \mathrm{H}, \mathrm{s})$, $3.51(1 \mathrm{H}, \mathrm{dd}, J=9.3,9.6 \mathrm{~Hz}), 3.53(1 \mathrm{H}, \mathrm{dd}, J=9.2,9.2$ $\mathrm{Hz}), 3.64(3 \mathrm{H}, \mathrm{s}), 3.67(1 \mathrm{H}, \mathrm{ddd}, J=3.7,3.9,9.4 \mathrm{~Hz})$, $3.76(1 \mathrm{H}, \mathrm{dd}, J=3.7,11.9 \mathrm{~Hz}), 3.78(1 \mathrm{H}, \mathrm{dd}, J=3.9$, $11.9 \mathrm{~Hz}), 4.61(1 \mathrm{H}, \mathrm{d}, J=12.2 \mathrm{~Hz}), 4.74(1 \mathrm{H}, \mathrm{d}, J=12.2$ $\mathrm{Hz}), 5.00(1 \mathrm{H}, \mathrm{d}, J=3.7 \mathrm{~Hz}), 7.30(1 \mathrm{H}, \mathrm{dd}, J=7.0,7.2$ $\mathrm{Hz}), 7.35(2 \mathrm{H}, \mathrm{d}, J=7.1,7.6 \mathrm{~Hz}), 7.39(2 \mathrm{H}, \mathrm{d}, J=7.2$ $\mathrm{Hz}) ;{ }^{13} \mathrm{C}$ NMR $\left(125.7 \mathrm{MHz}, \mathrm{CDCl}_{3}\right) \delta 58.0\left(\mathrm{CH}_{3}\right), 61.2$ $\left(\mathrm{CH}_{3}\right), 62.3\left(\mathrm{CH}_{2}\right), 69.3\left(\mathrm{CH}_{2}\right), 70.4(\mathrm{CH}), 71.0(\mathrm{CH})$, $81.8(\mathrm{CH}), 82.6(\mathrm{CH}), 95.0(\mathrm{CH}), 128.0(\mathrm{CH}), 128.3(2 \times$ $\mathrm{CH}), 128.4(2 \times \mathrm{CH}), 137.0(\mathrm{C})$. MS $\mathrm{m} / z$ (rel intensity) $280\left(\mathrm{M}^{+}-\mathrm{H}_{2} \mathrm{O},<1\right), 91\left(\mathrm{PhCH}_{2}, 100\right)$; HRMS calcd for $\mathrm{C}_{15} \mathrm{H}_{20} \mathrm{O}_{5}$ 280.1311, found 280.1297. Anal. Calcd for $\mathrm{C}_{15} \mathrm{H}_{22} \mathrm{O}_{6}$ : C, 60.39; H, 7.43. Found: C, 60.33; H, 7.68.

Benzyl 2,6-Di- $O$-methyl- $\beta$-D-glucopyranoside (32): IR $\left(\mathrm{CHCl}_{3}\right)$ 3592, $3461 \mathrm{~cm}^{-1}$; ${ }^{1} \mathrm{H}$ NMR $(500 \mathrm{MHz}$, $\left.\mathrm{CDCl}_{3}\right) \delta 2.93(1 \mathrm{H}, \mathrm{br} \mathrm{s}), 3.06(1 \mathrm{H}, \mathrm{dd}, J=7.8,8.9 \mathrm{~Hz})$, $3.40(1 \mathrm{H}, \mathrm{m}), 3.43(3 \mathrm{H}, \mathrm{s}), 3.49(1 \mathrm{H}, \mathrm{m}), 3.57(1 \mathrm{H}, \mathrm{dd}, J$ $=8.5,10.0 \mathrm{~Hz}), 3.61(3 \mathrm{H}, \mathrm{s}), 3.69(2 \mathrm{H}, \mathrm{d}, J=4.7 \mathrm{~Hz})$, $4.43(1 \mathrm{H}, \mathrm{d}, J=7.8 \mathrm{~Hz}), 4.63(1 \mathrm{H}, \mathrm{d}, J=11.9 \mathrm{~Hz}), 4.93$ $(1 \mathrm{H}, \mathrm{d}, J=11.9 \mathrm{~Hz}), 7.34-7.44(5 \mathrm{H}, \mathrm{m}) ;{ }^{13} \mathrm{C}$ NMR $(100.6$ $\left.\mathrm{MHz}, \mathrm{CDCl}_{3}\right) \delta 59.6\left(\mathrm{CH}_{3}\right), 60.7\left(\mathrm{CH}_{3}\right), 71.0\left(\mathrm{CH}_{2}\right), 71.3$ $(\mathrm{CH}), 72.7\left(\mathrm{CH}_{2}\right), 73.8(\mathrm{CH}), 76.2(\mathrm{CH}), 82.8(\mathrm{CH})$, $102.3(\mathrm{CH}), 127.8(3 \times \mathrm{CH}), 128.4(2 \times \mathrm{CH}), 137.3(\mathrm{C})$. MS $m / z$ (rel intensity) $235\left(\mathrm{M}^{+}-\mathrm{MeOH}-\mathrm{MeO},<1\right), 91$ $\left(\mathrm{PhCH}_{2}, 100\right)$; HRMS calcd for $\mathrm{C}_{13} \mathrm{H}_{15} \mathrm{O}_{4}, 235.0970$, found 235.0975. Anal. Calcd for $\mathrm{C}_{15} \mathrm{H}_{22} \mathrm{O}_{6}: \mathrm{C}, 60.39 ; \mathrm{H}$, 7.43. Found: C, 60.46; H, 7.49. 
${ }^{1} \mathrm{H}$ and ${ }^{13} \mathrm{C}$ NMR spectra for compounds 12, 14-20, 22-27, and 29-33.

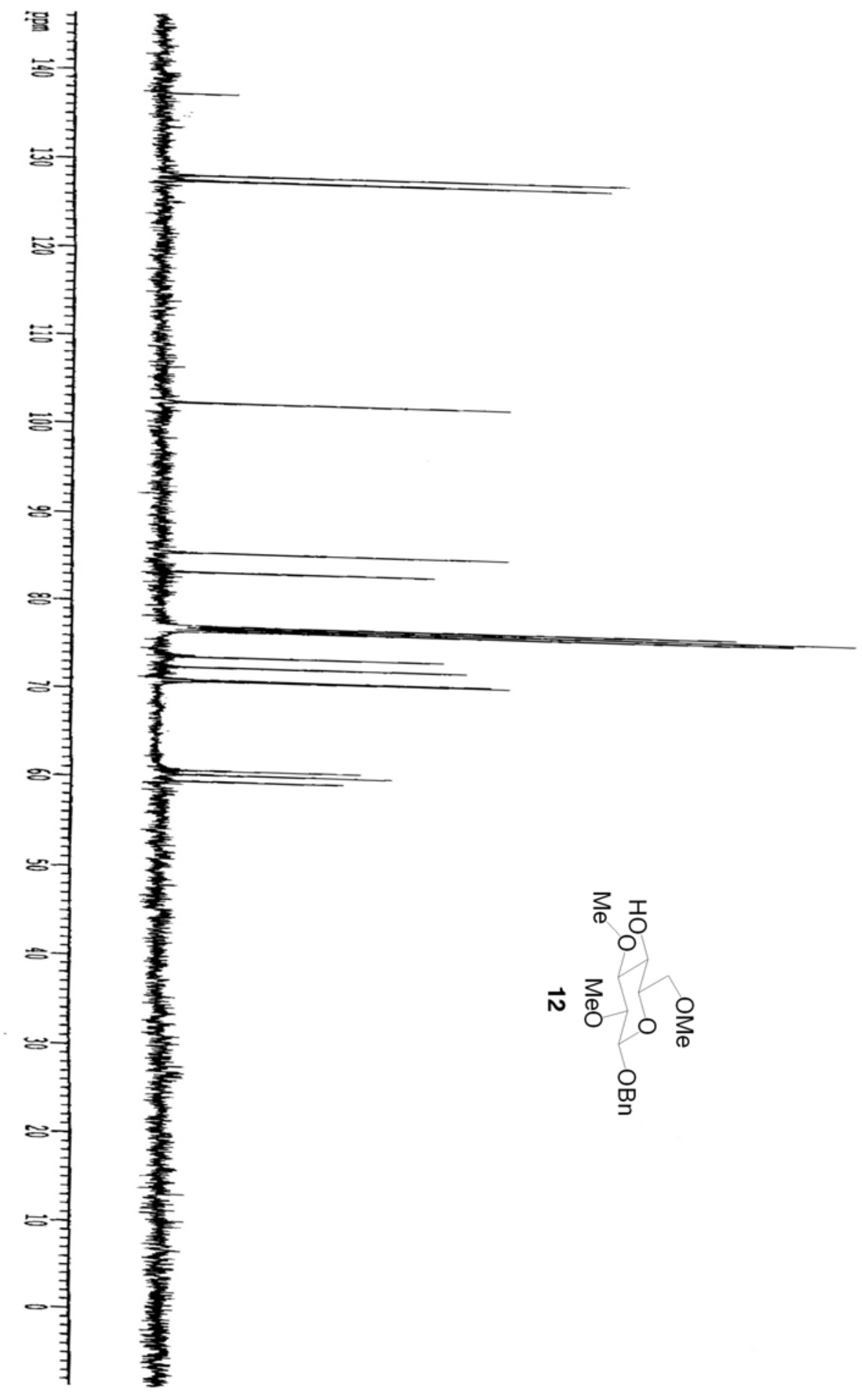




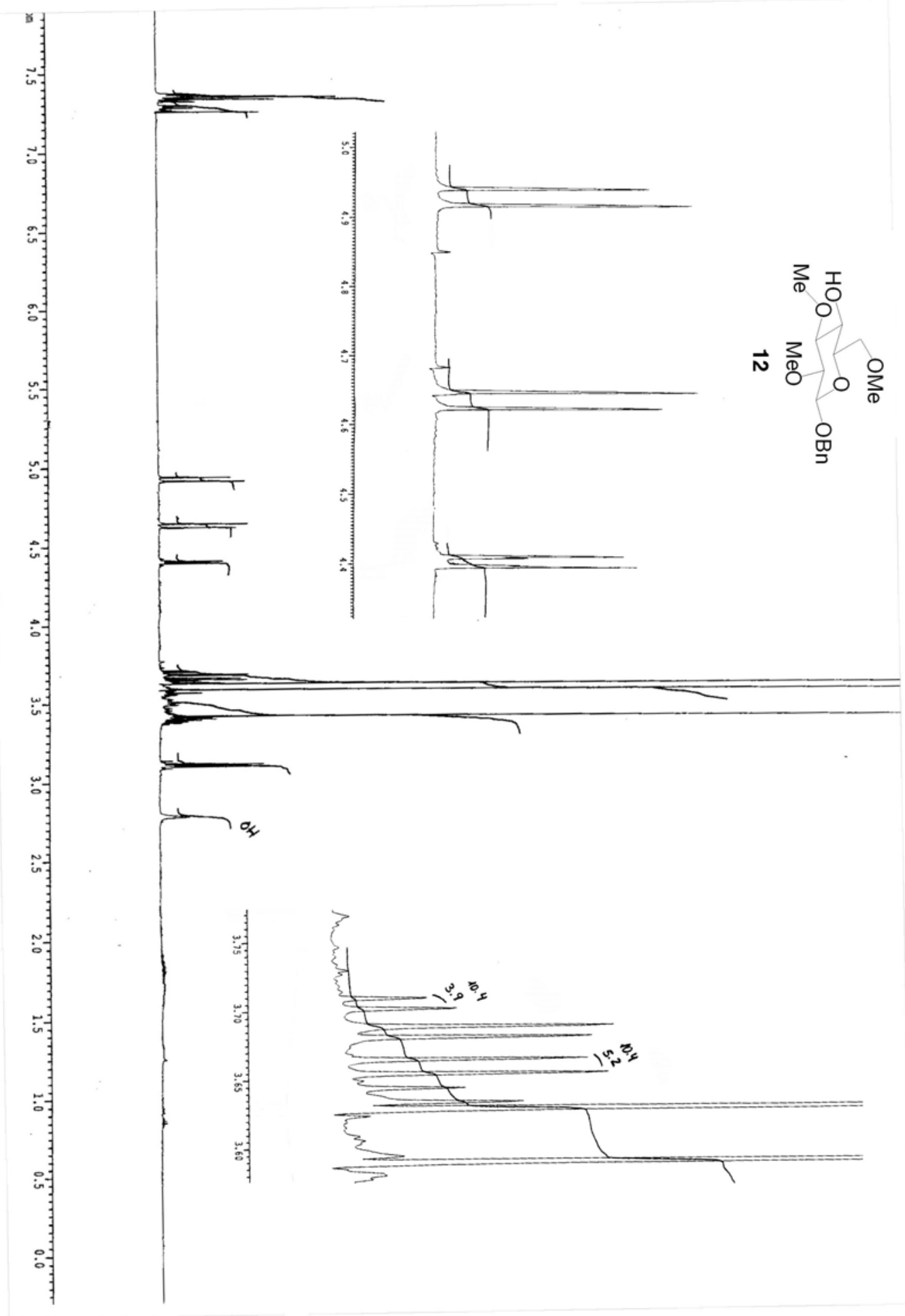




$$
F
$$




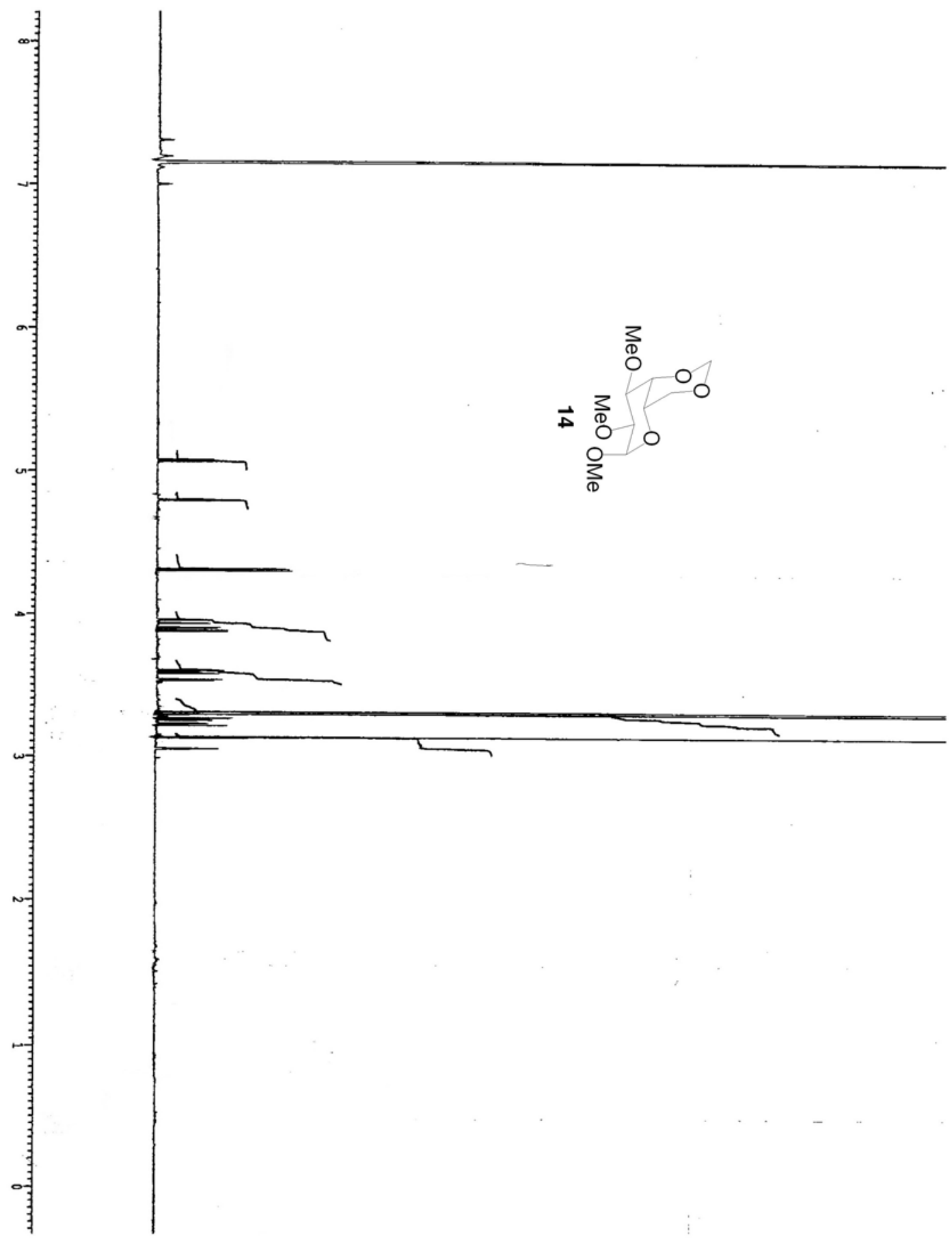




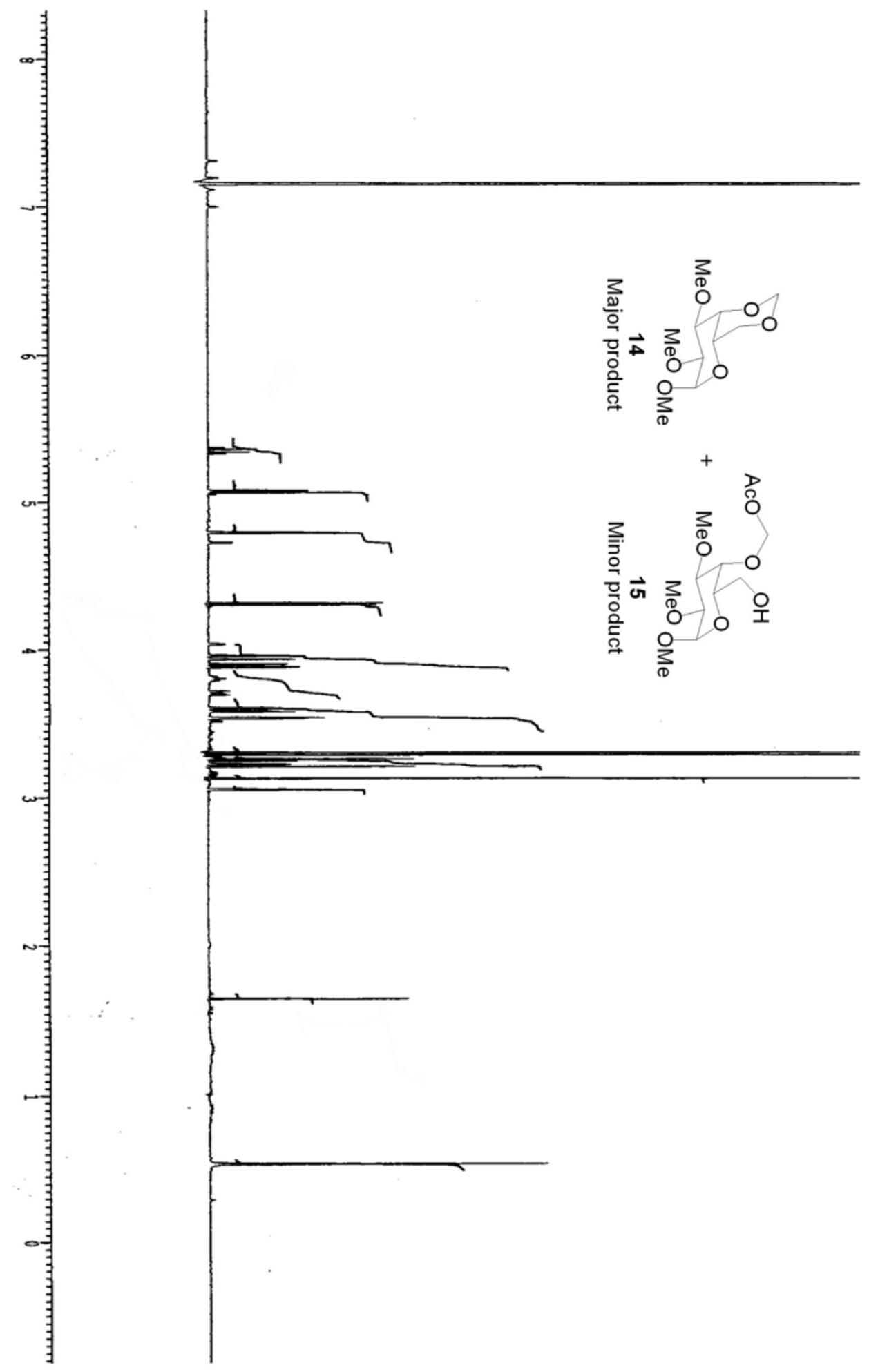




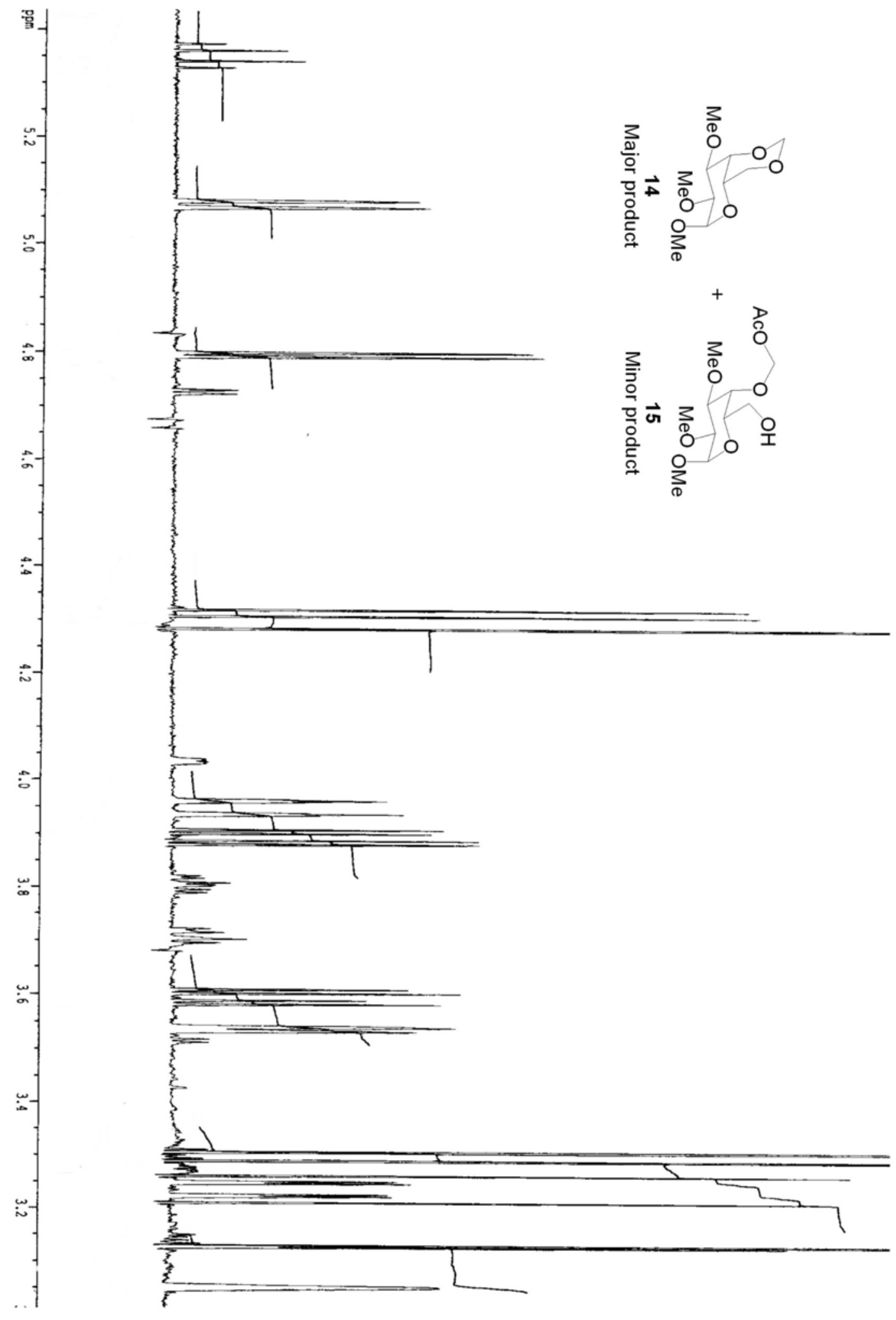




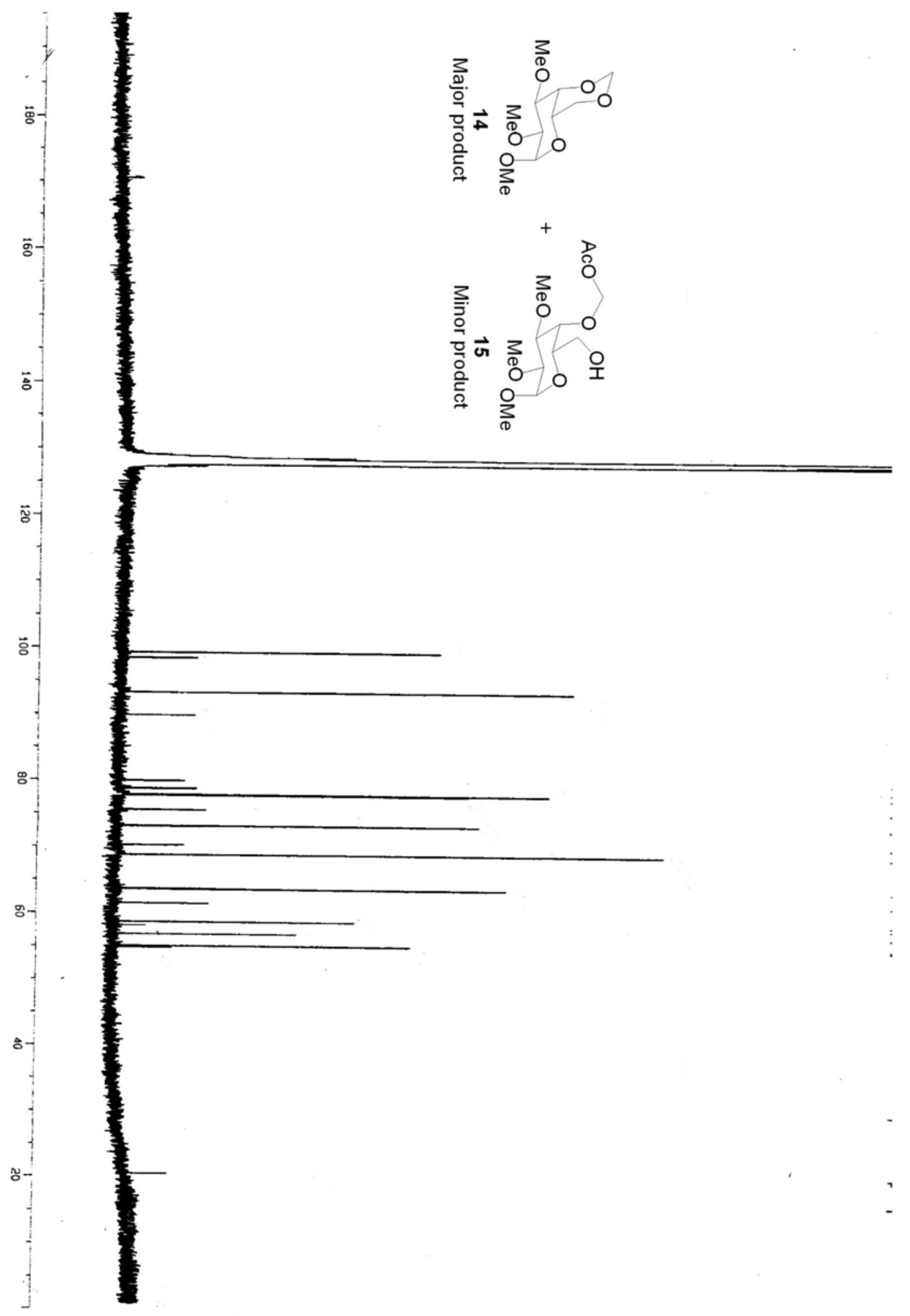

11 

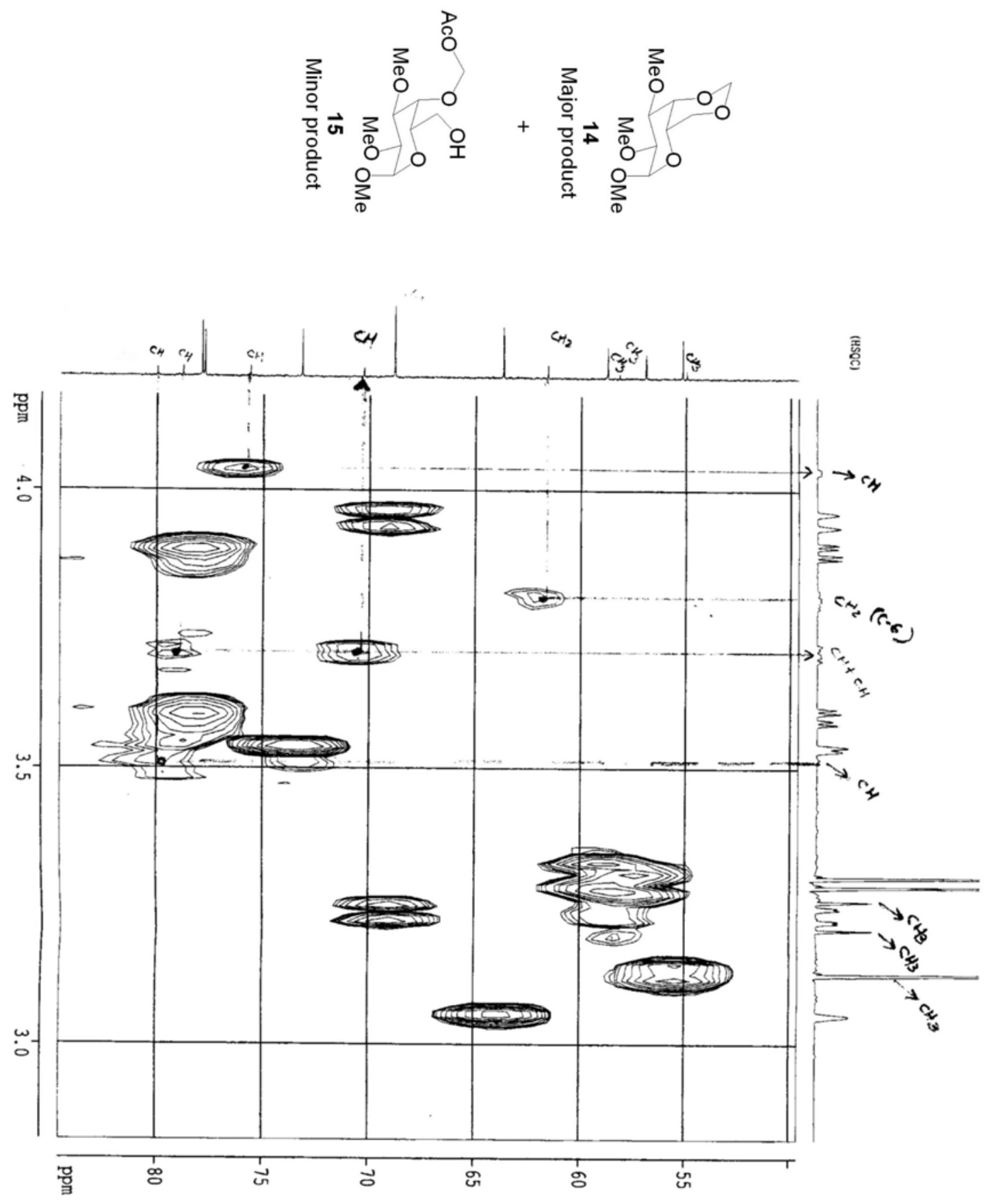


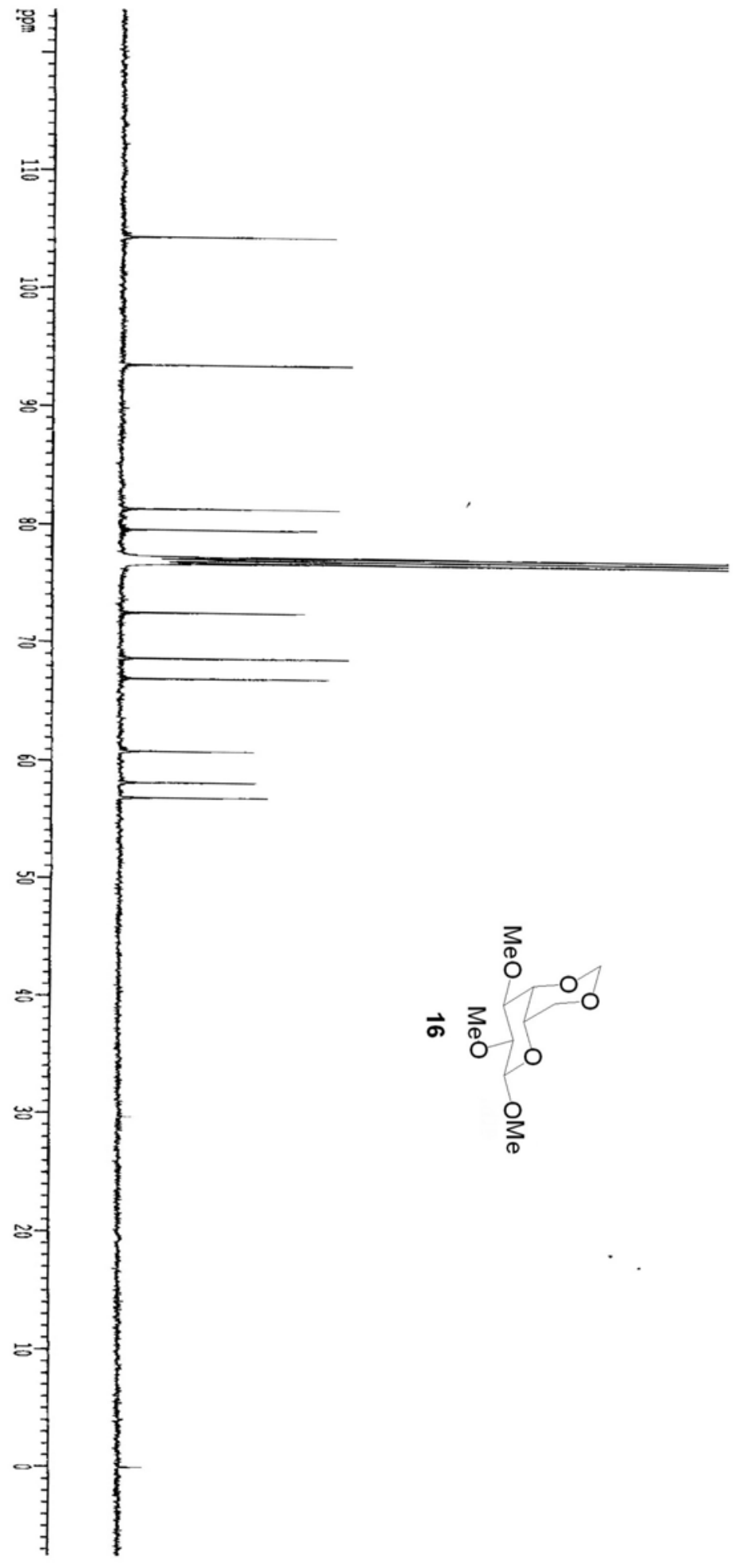




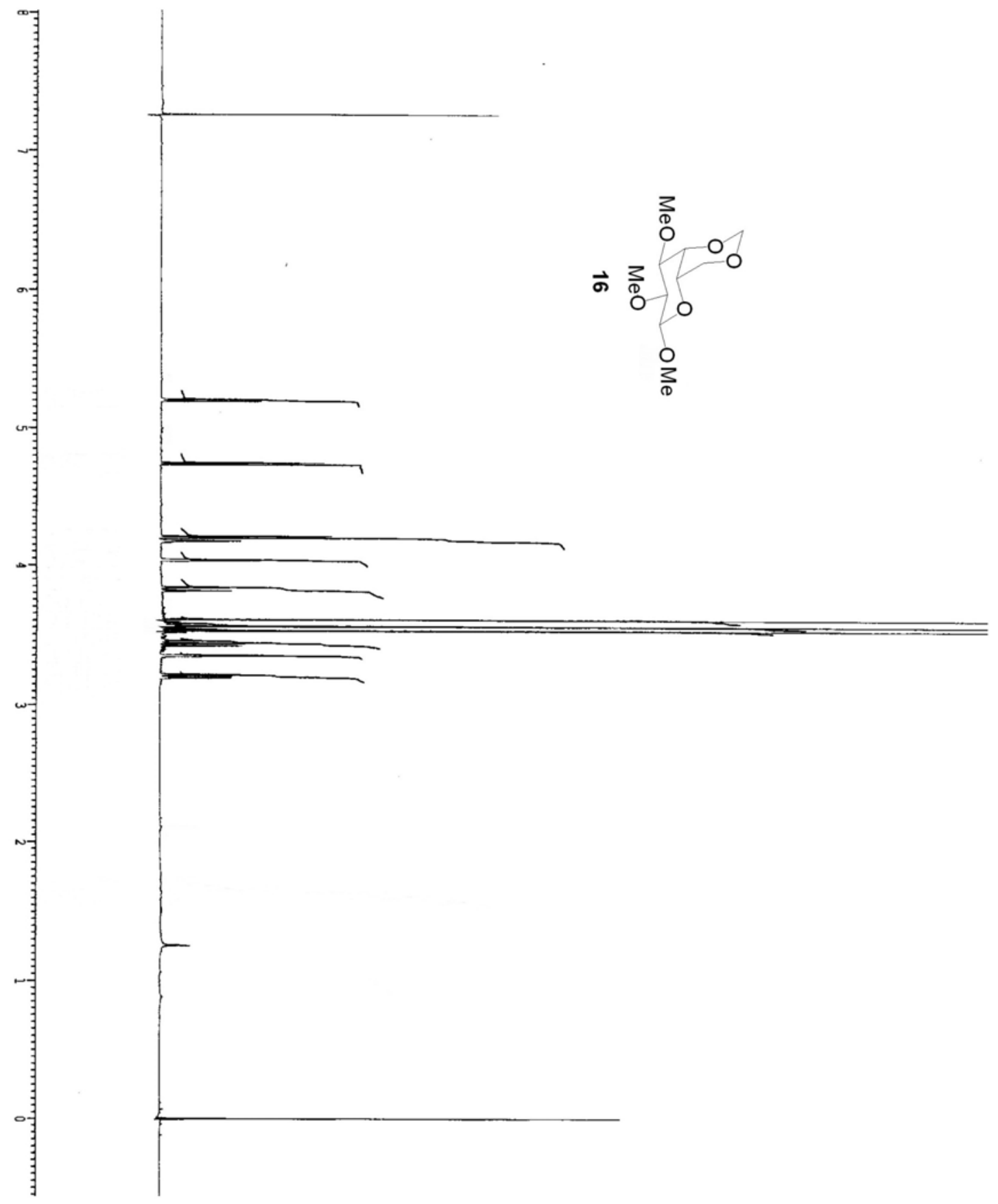




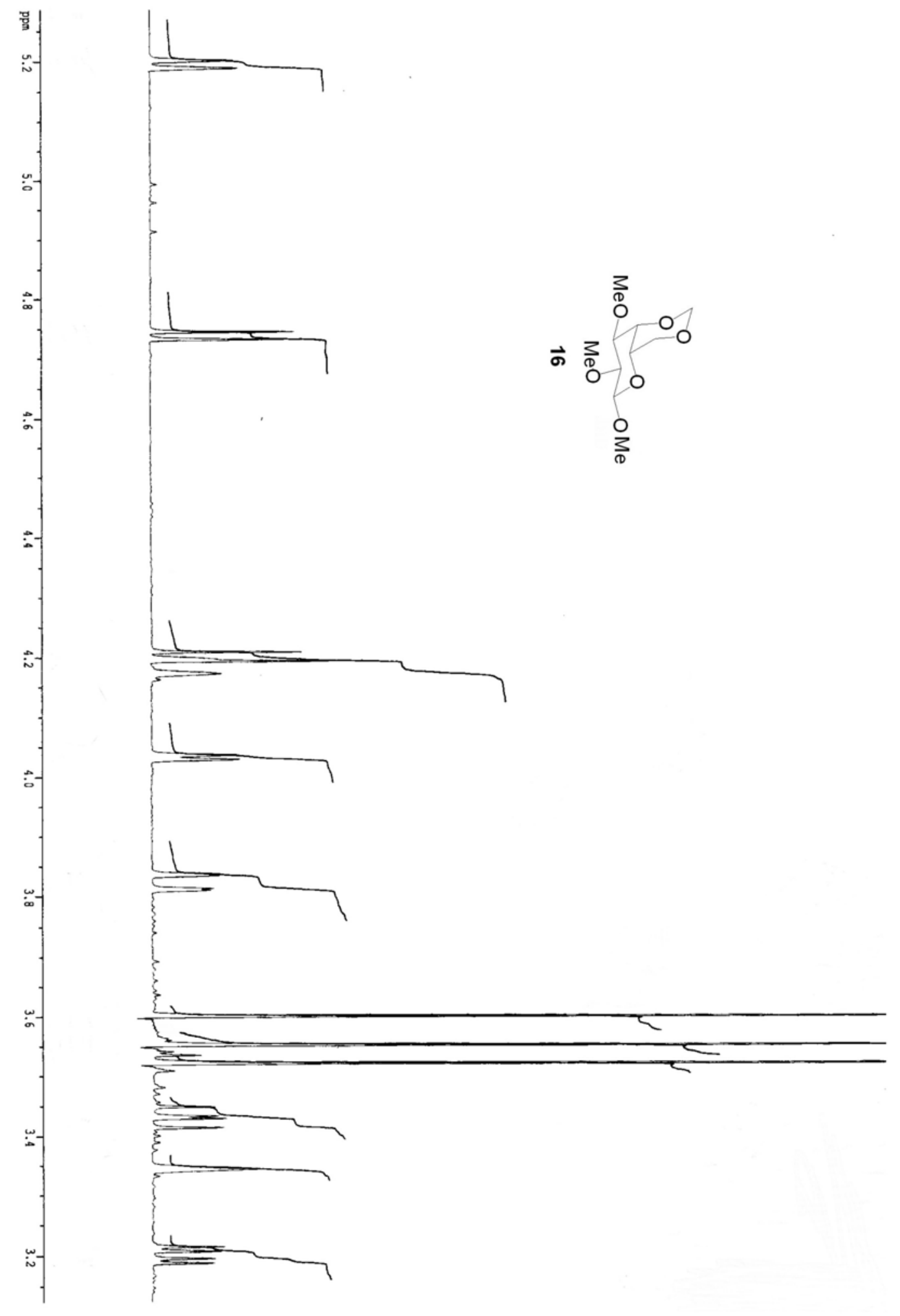




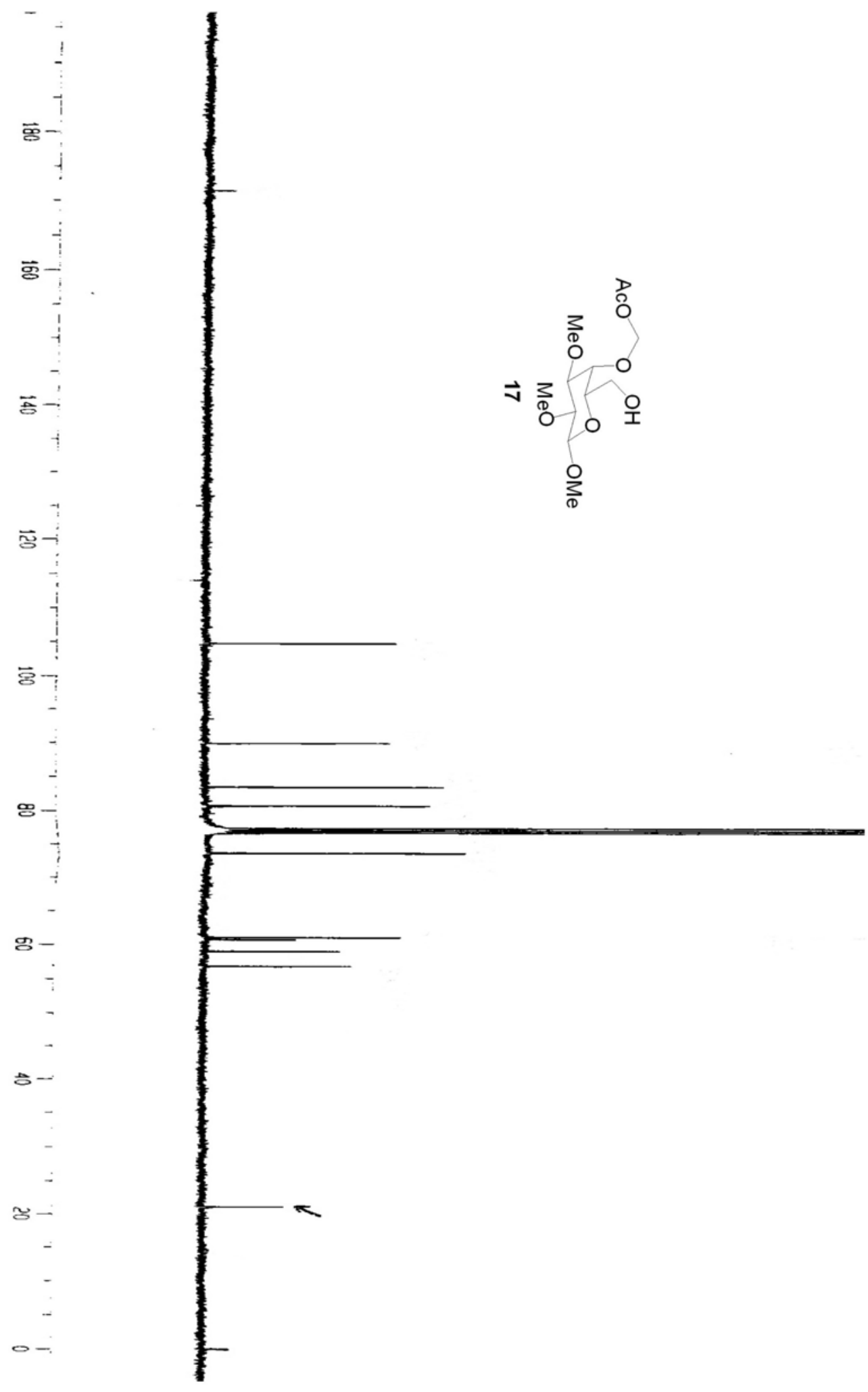




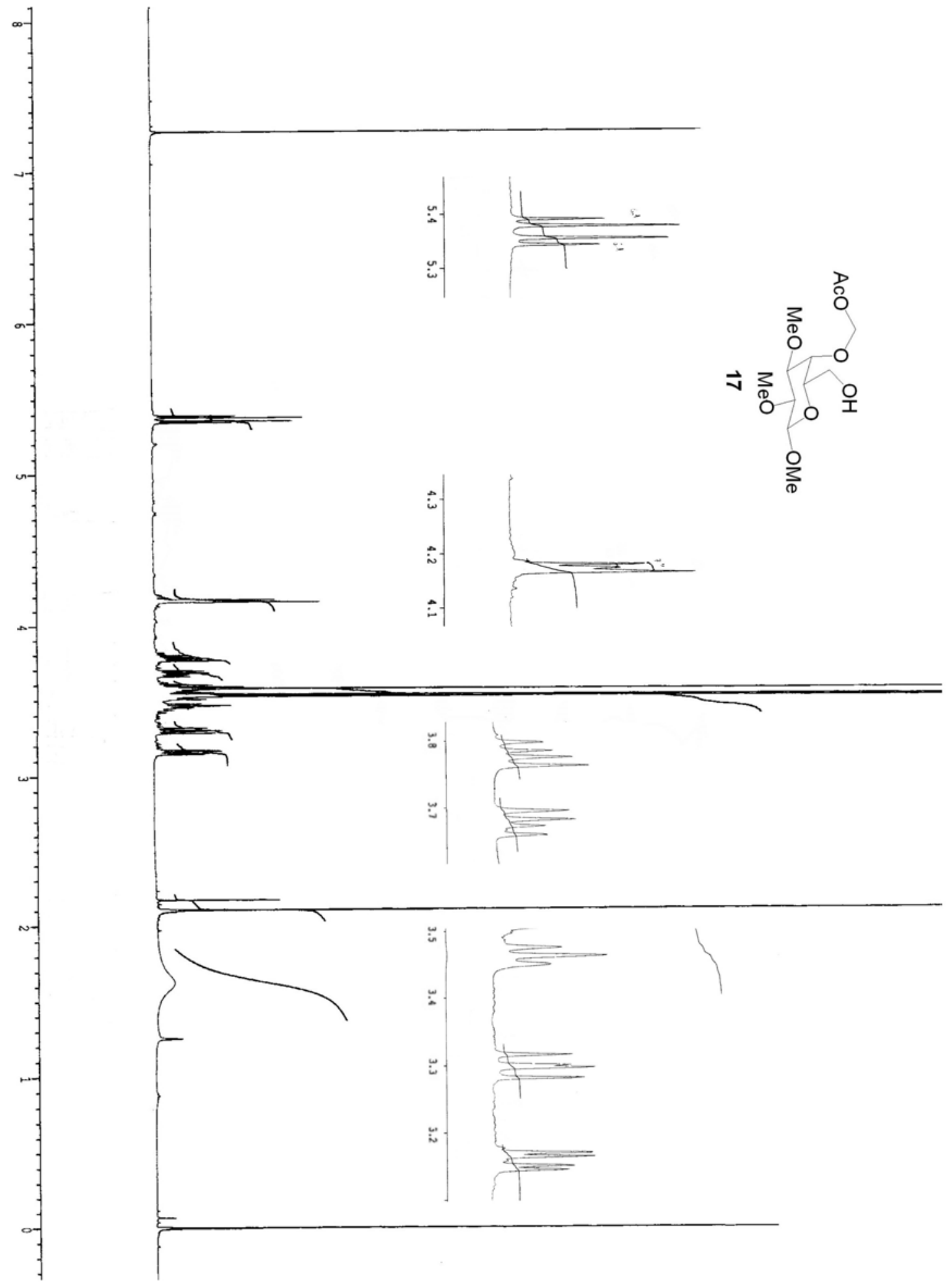




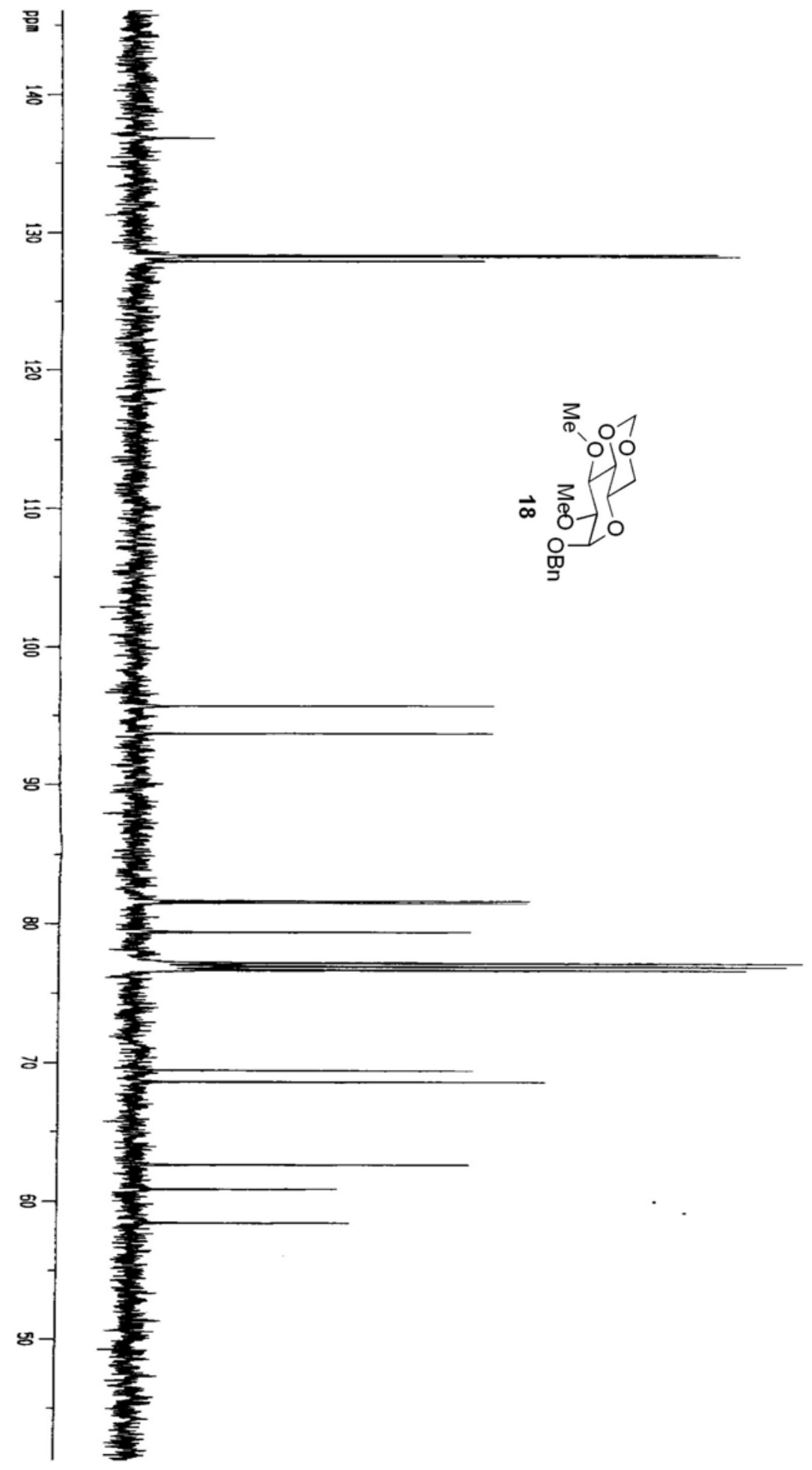




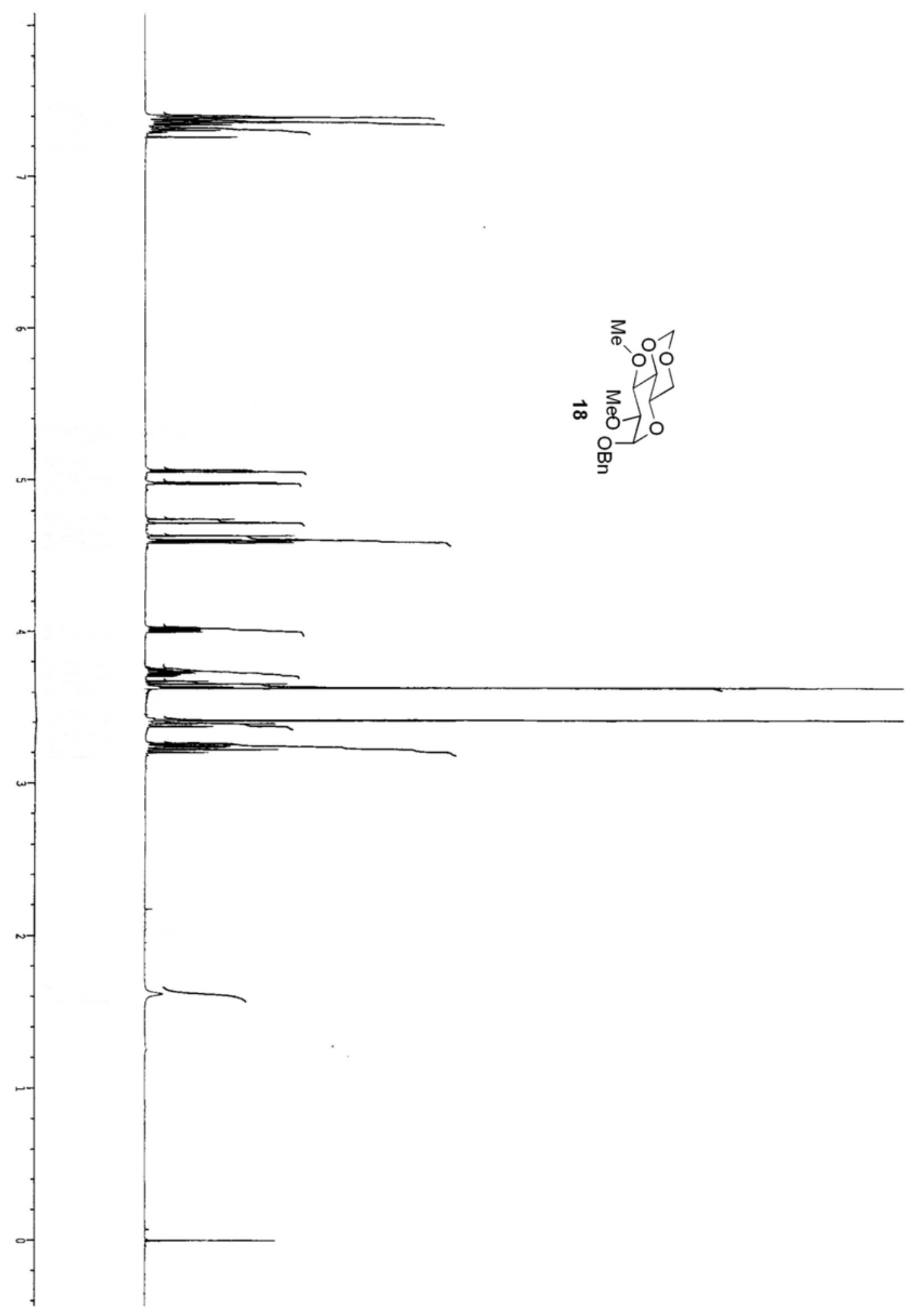

19 


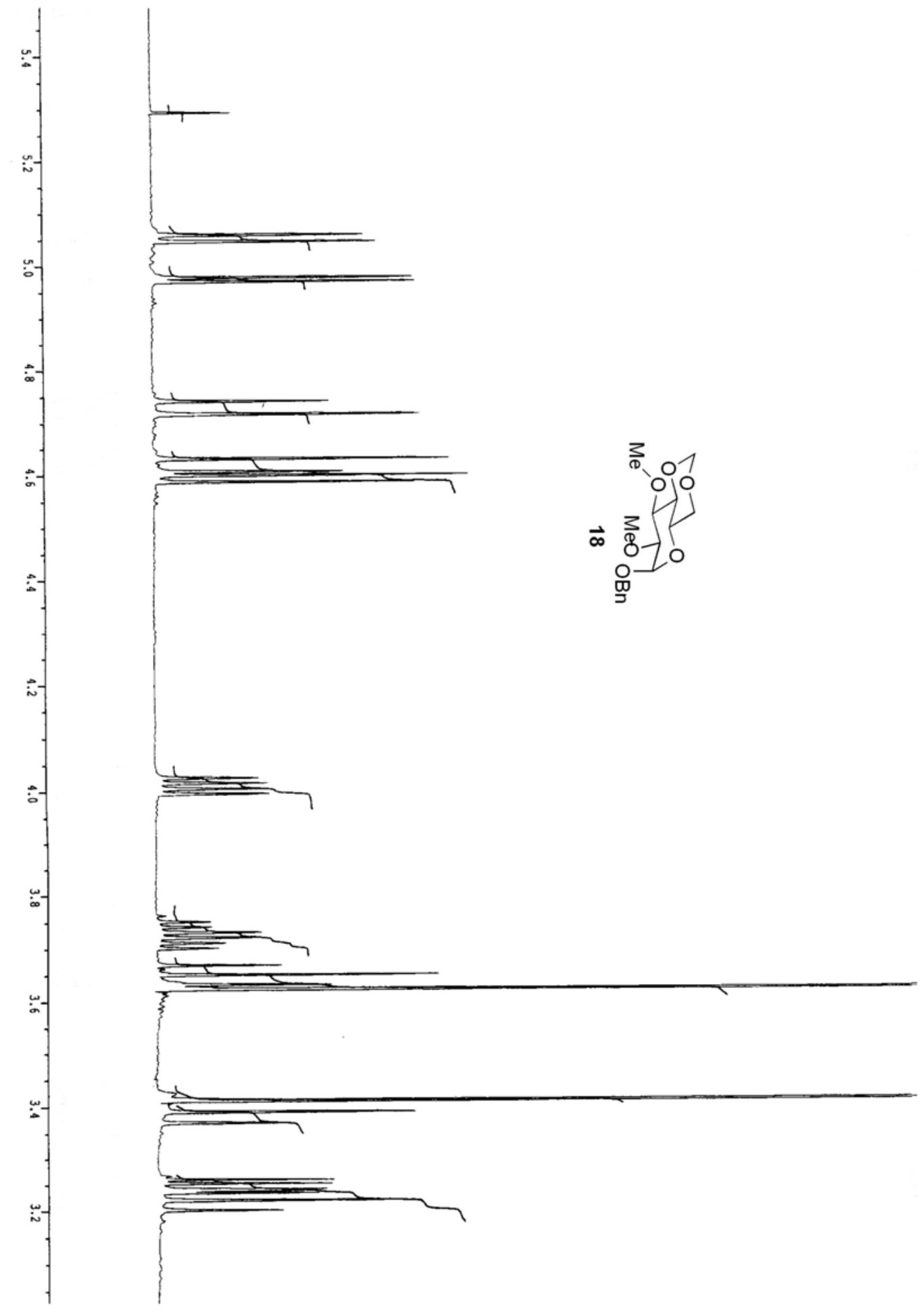




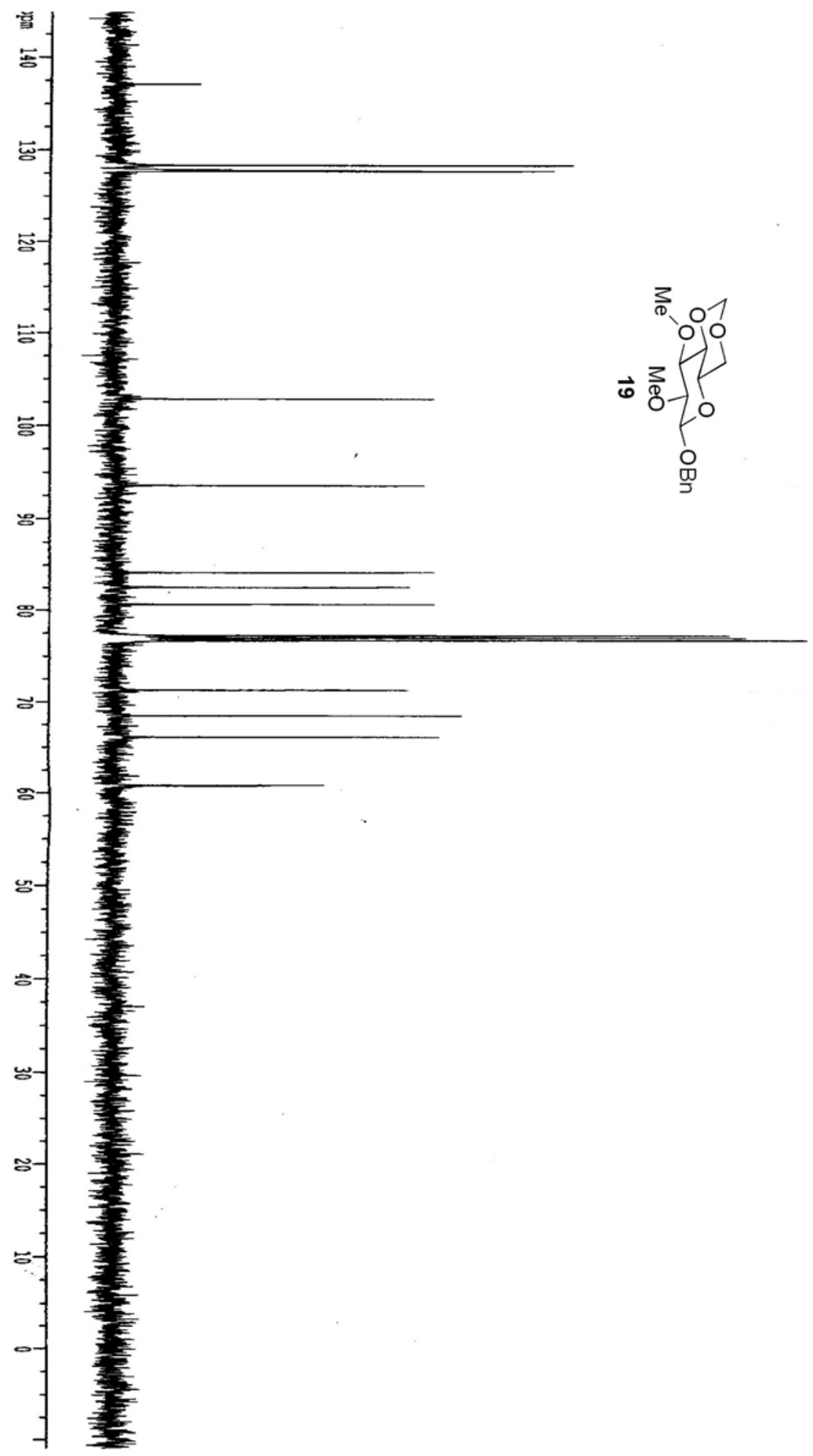




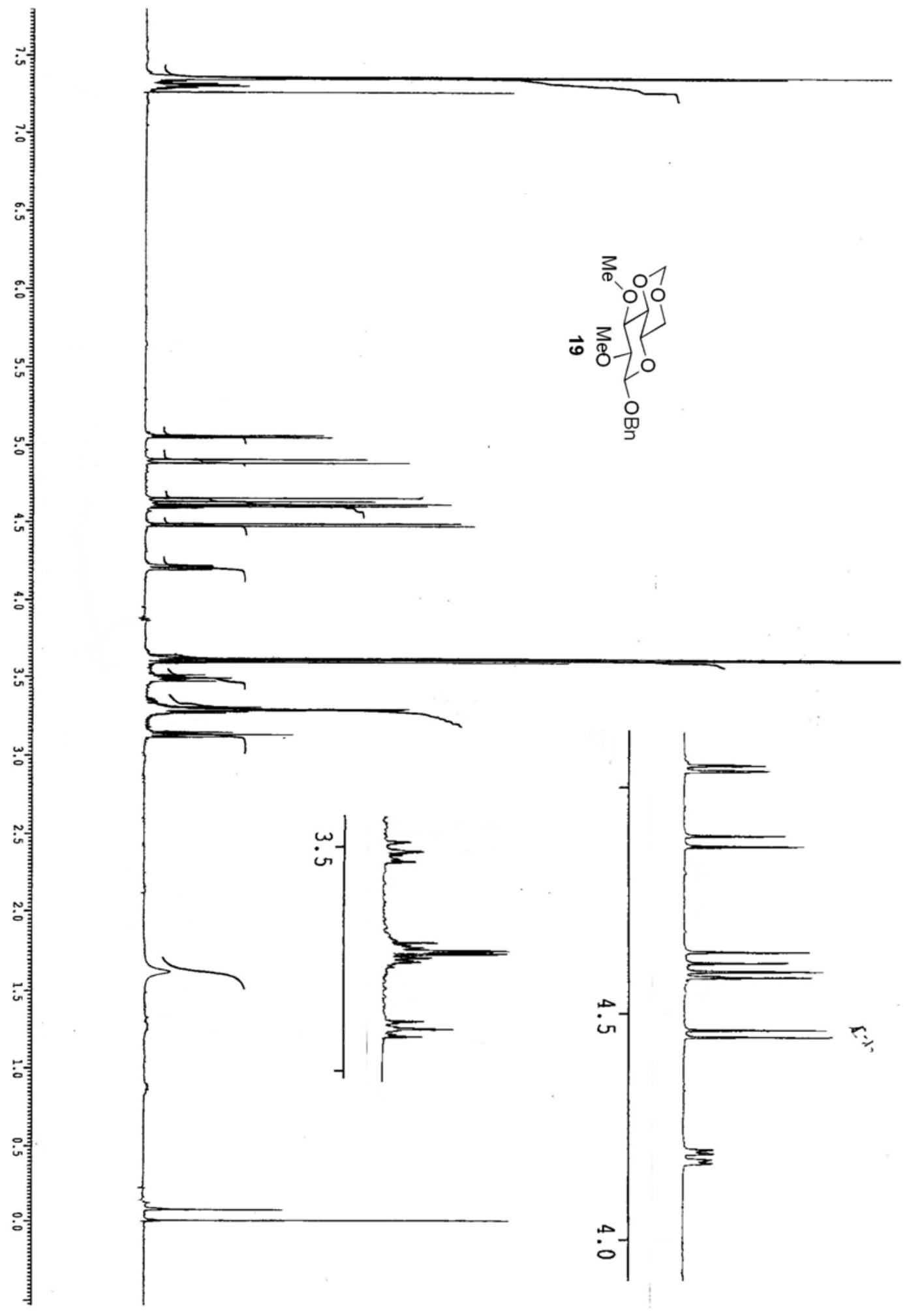




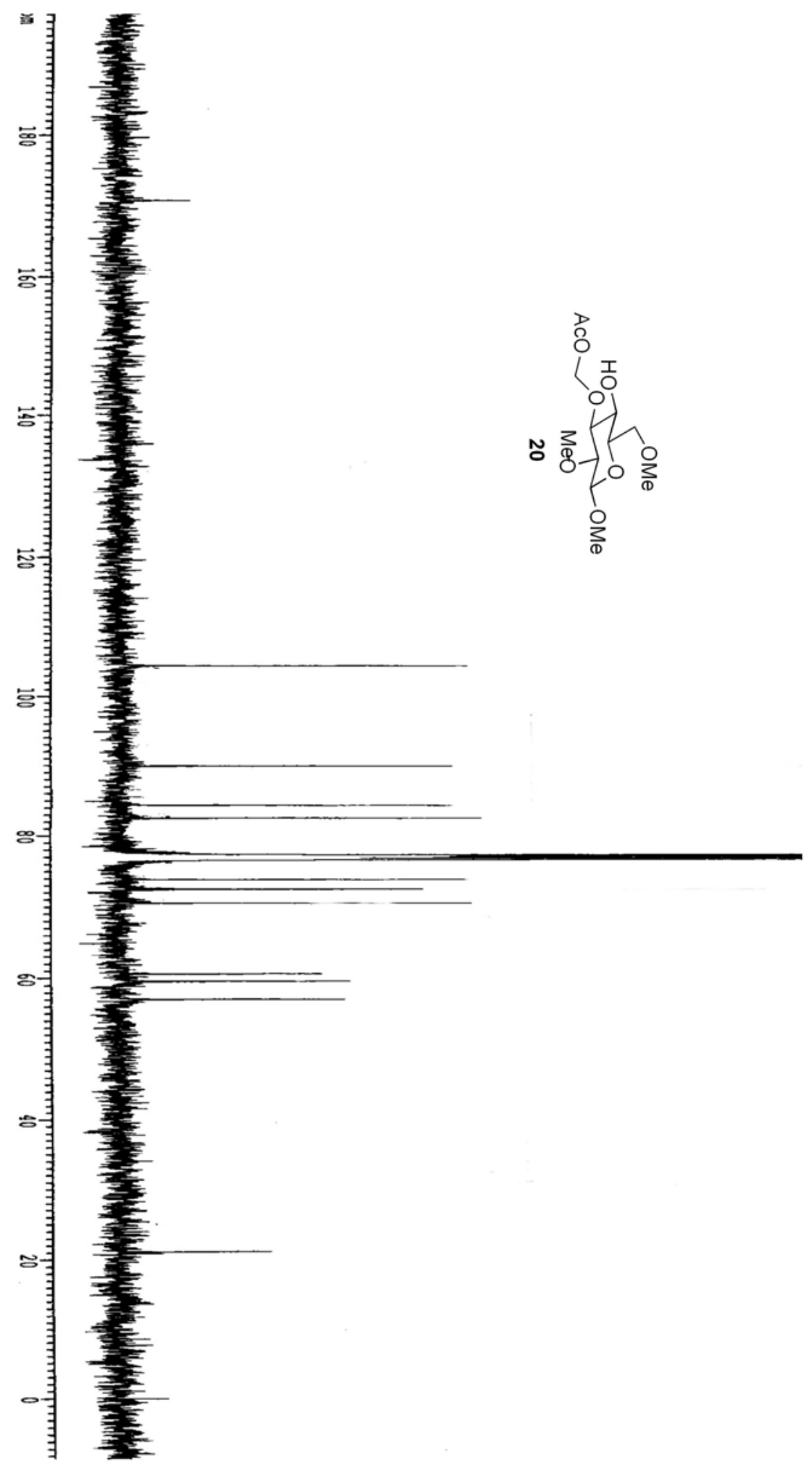




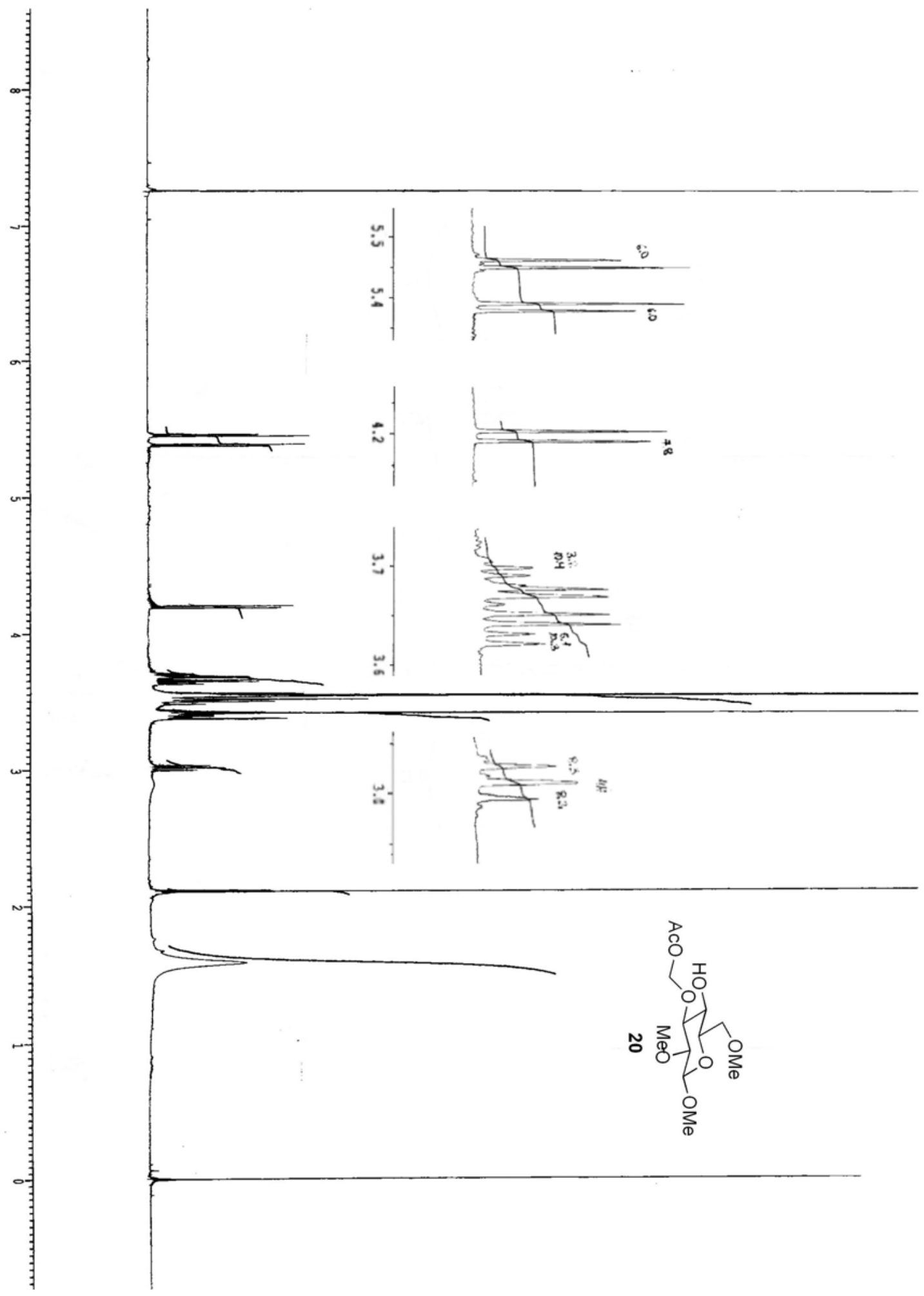




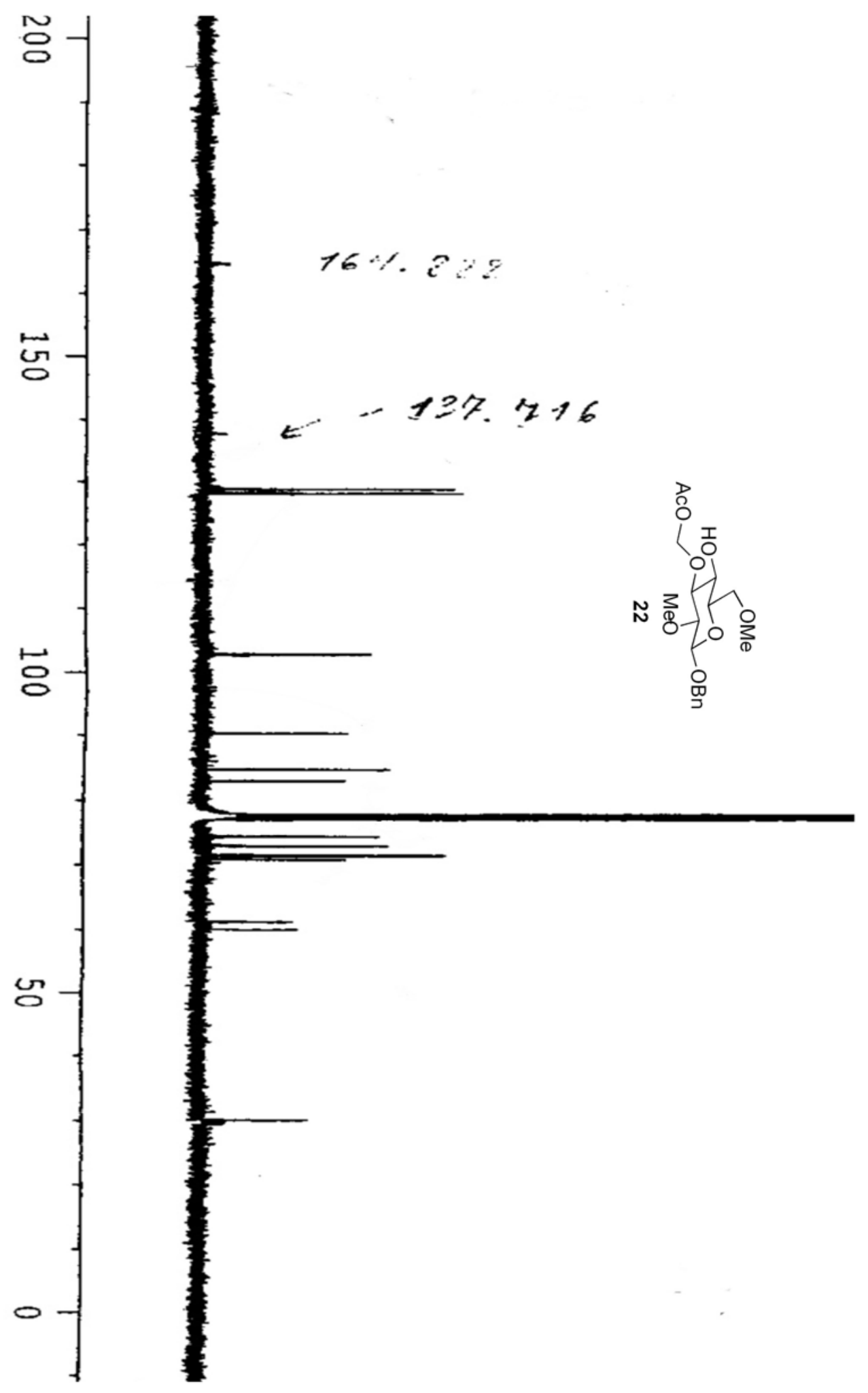




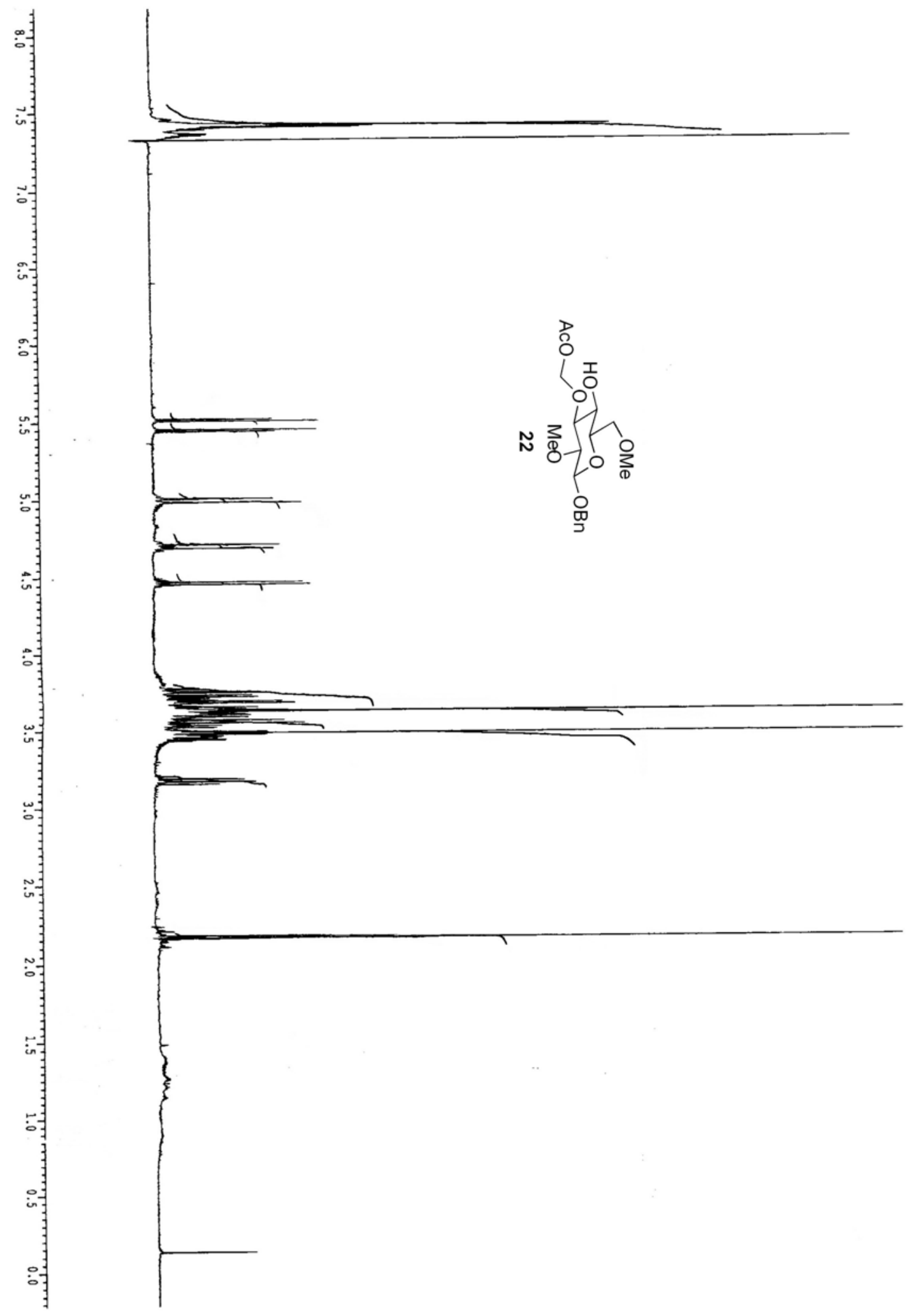



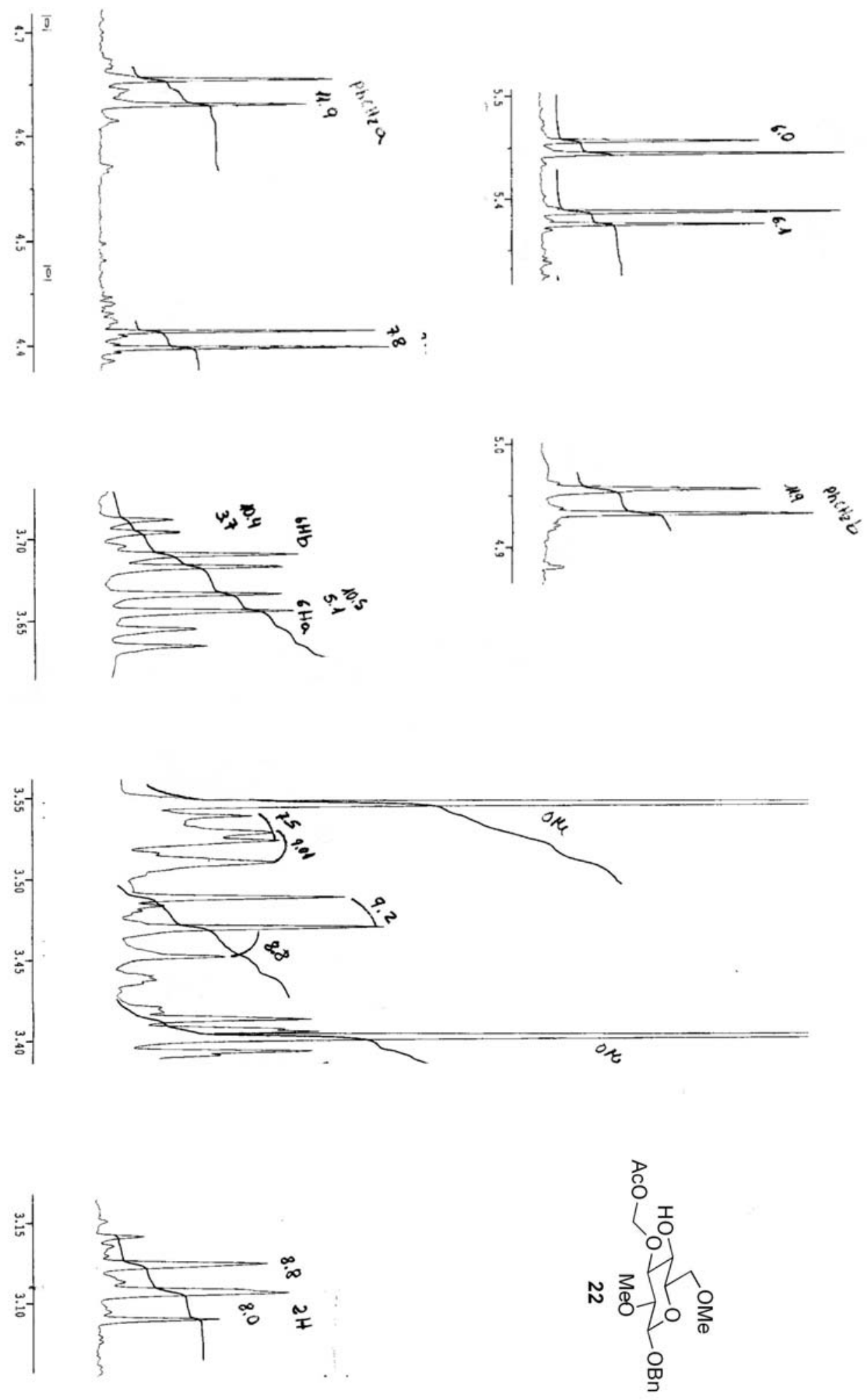


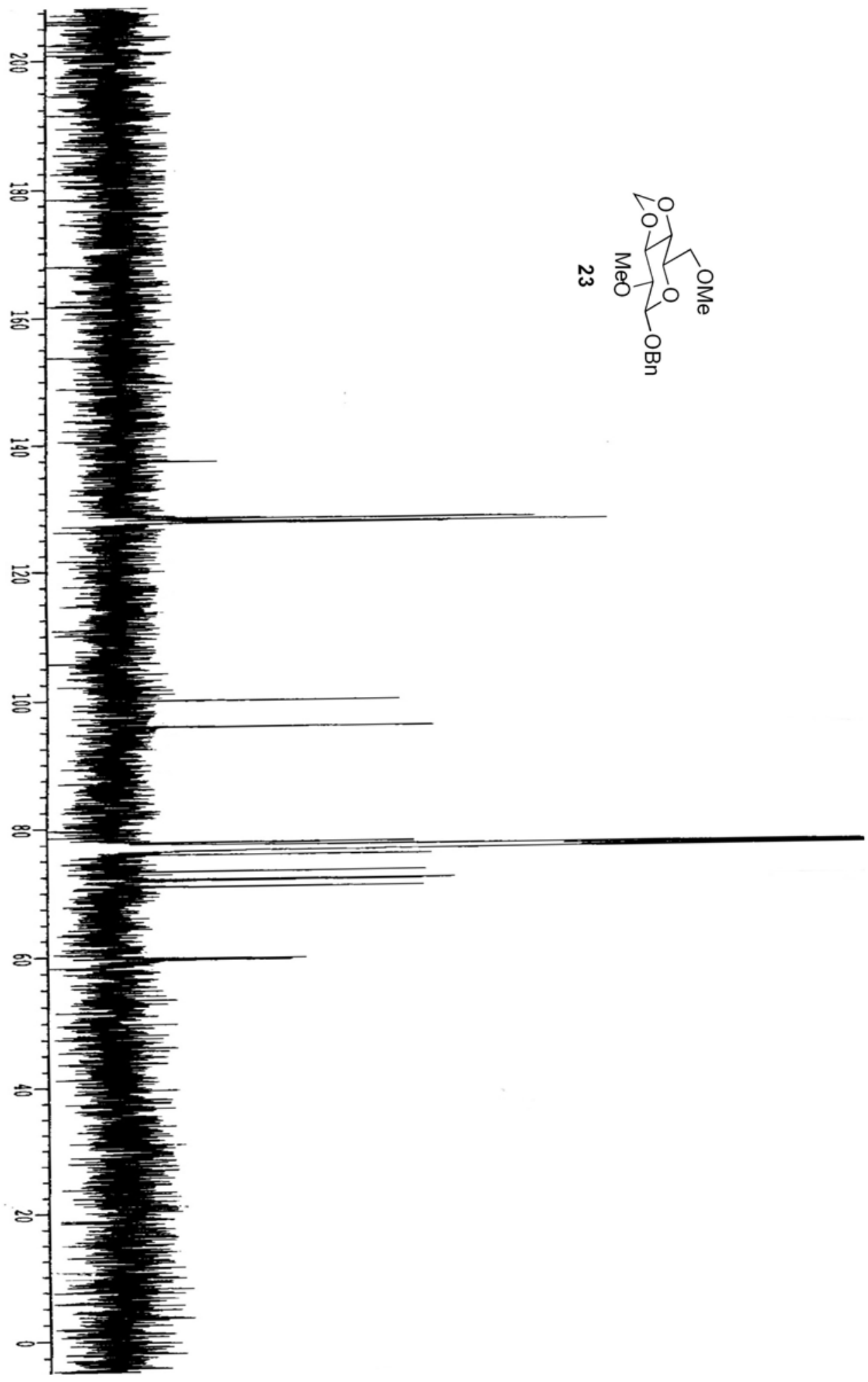




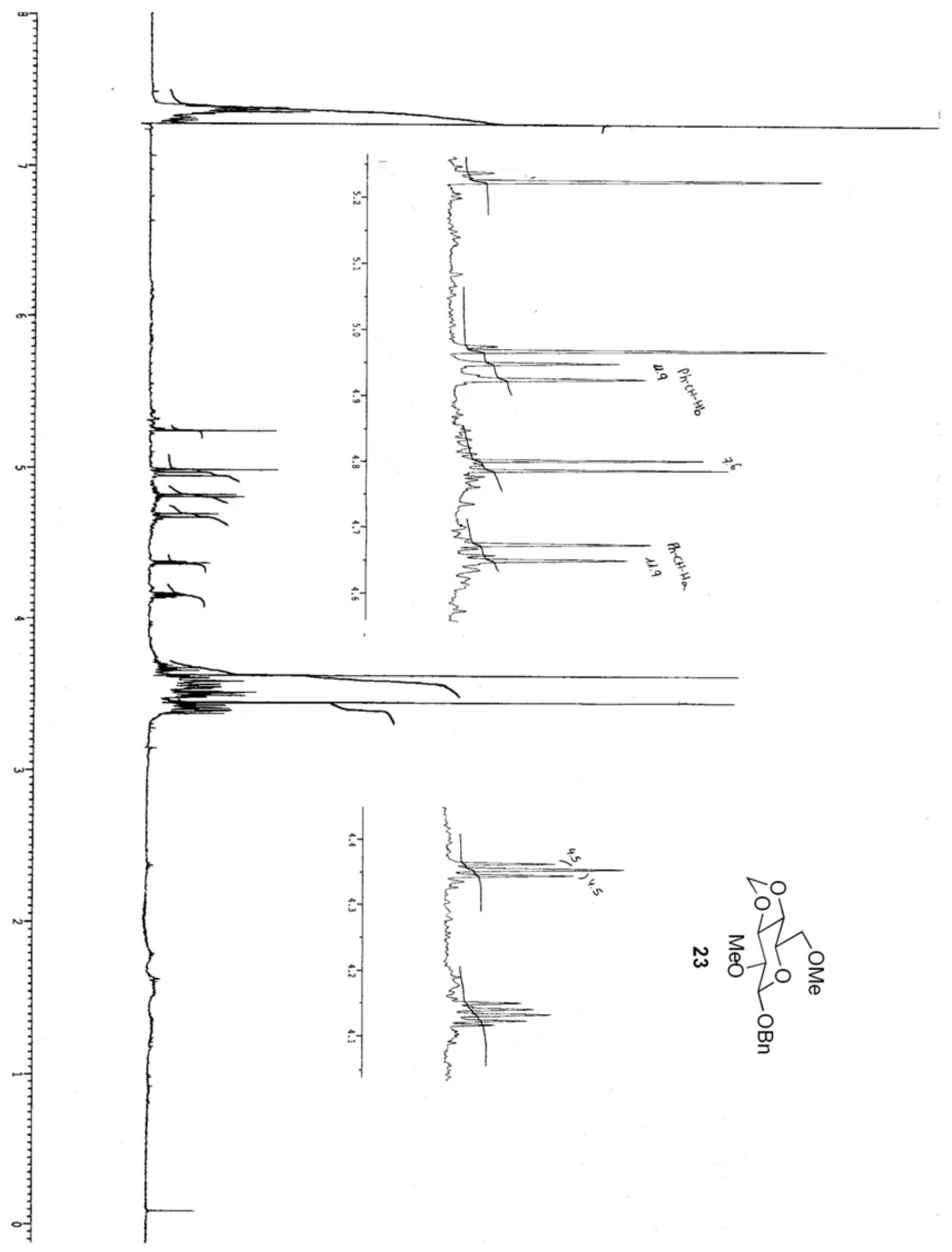




$$
H
$$




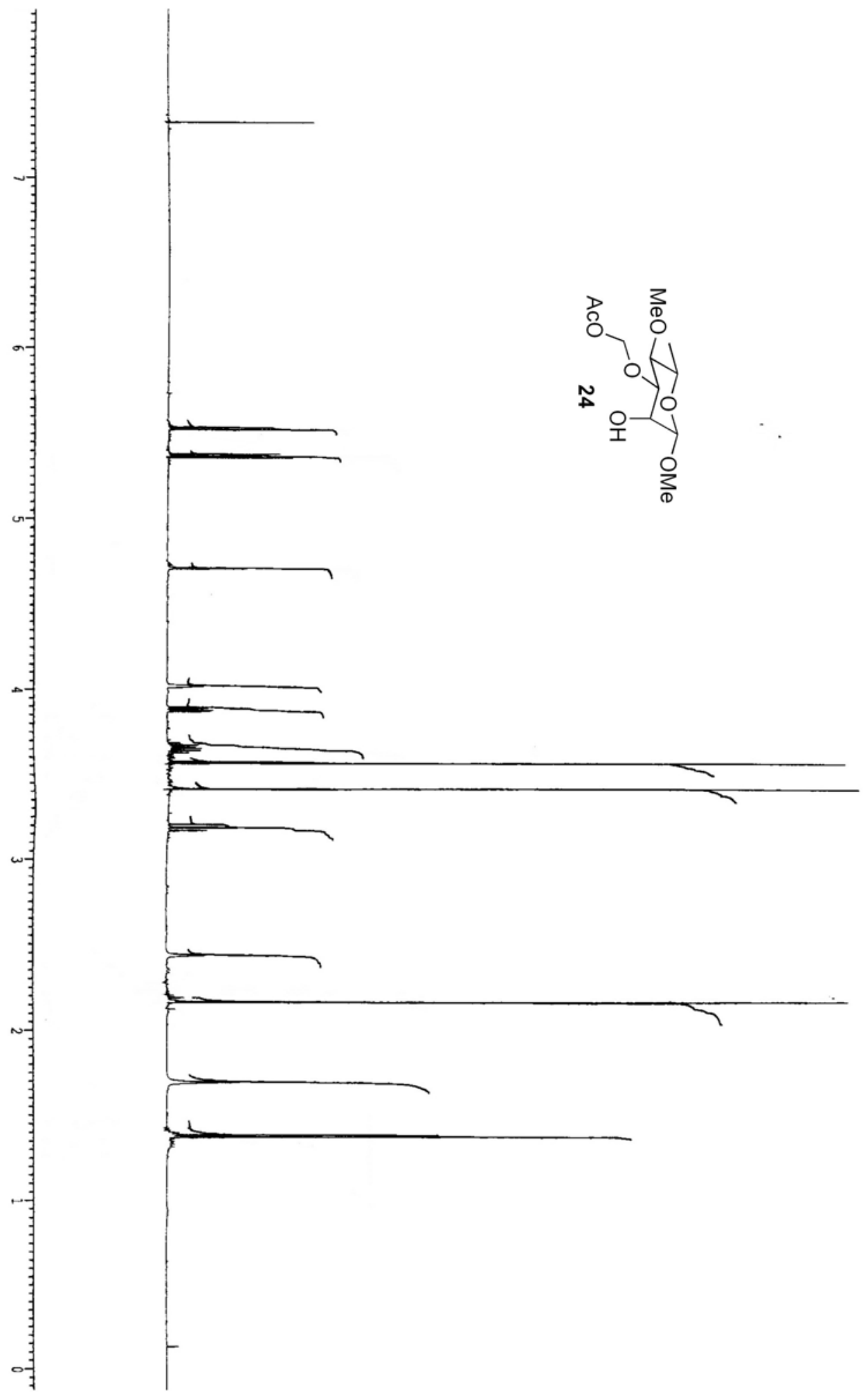




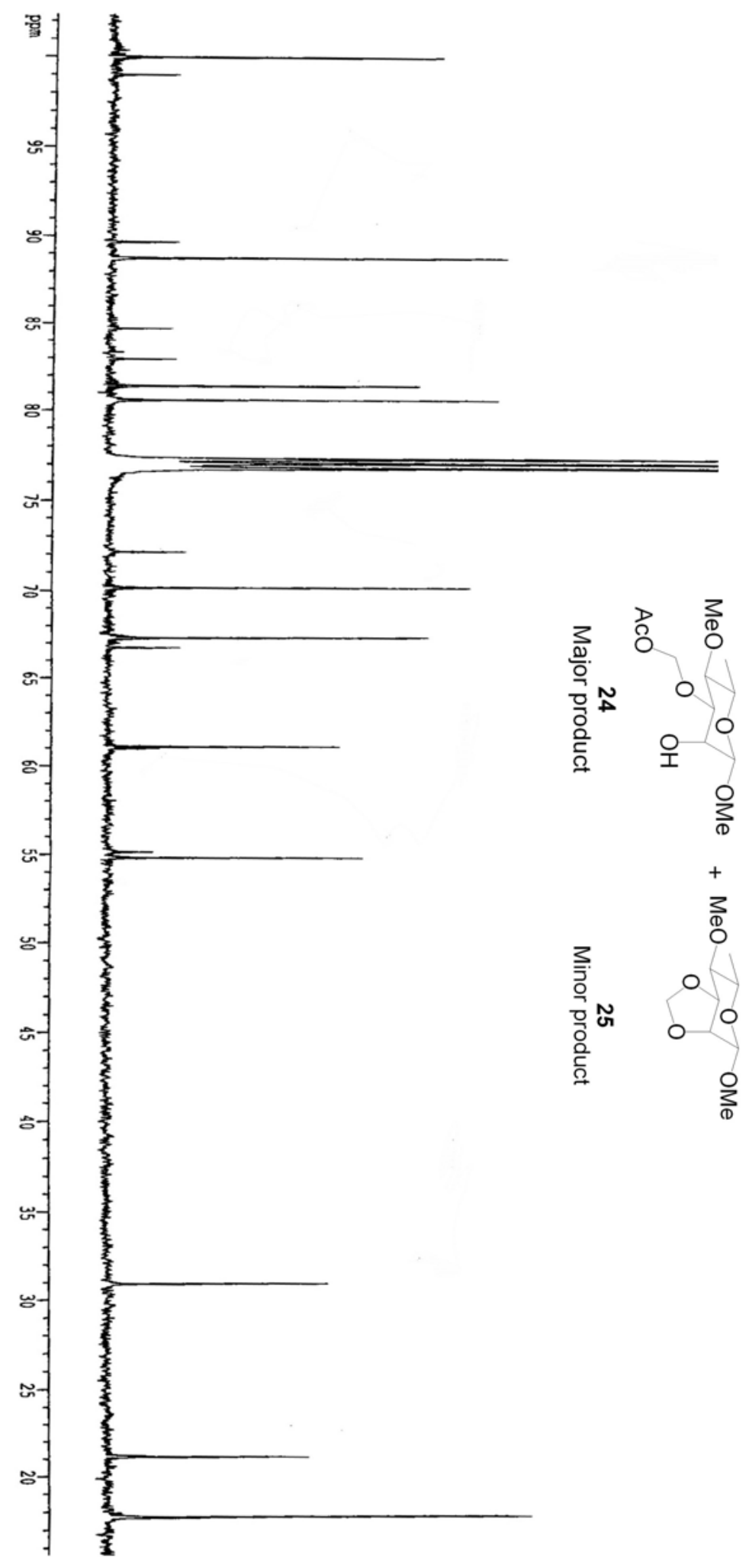




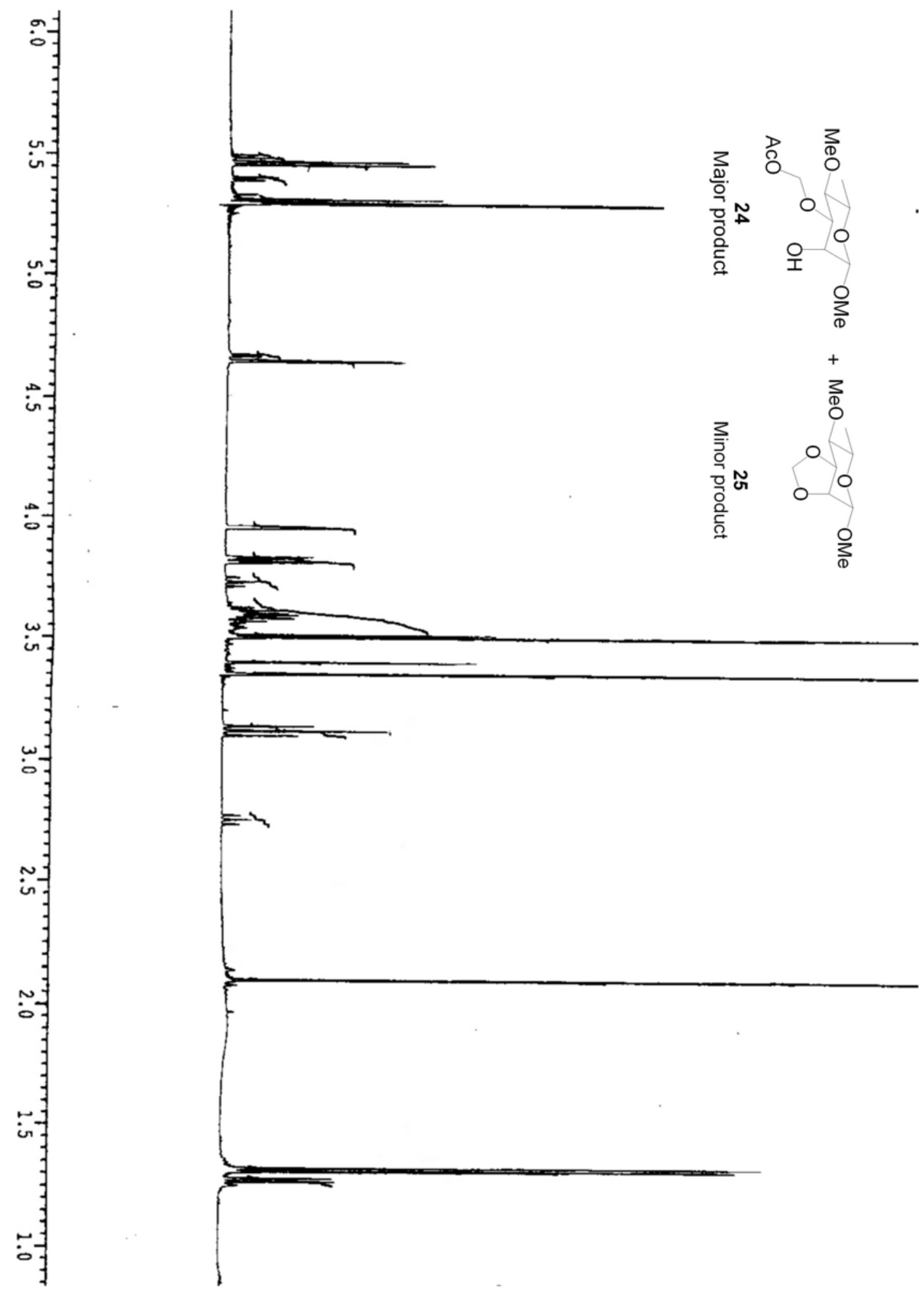




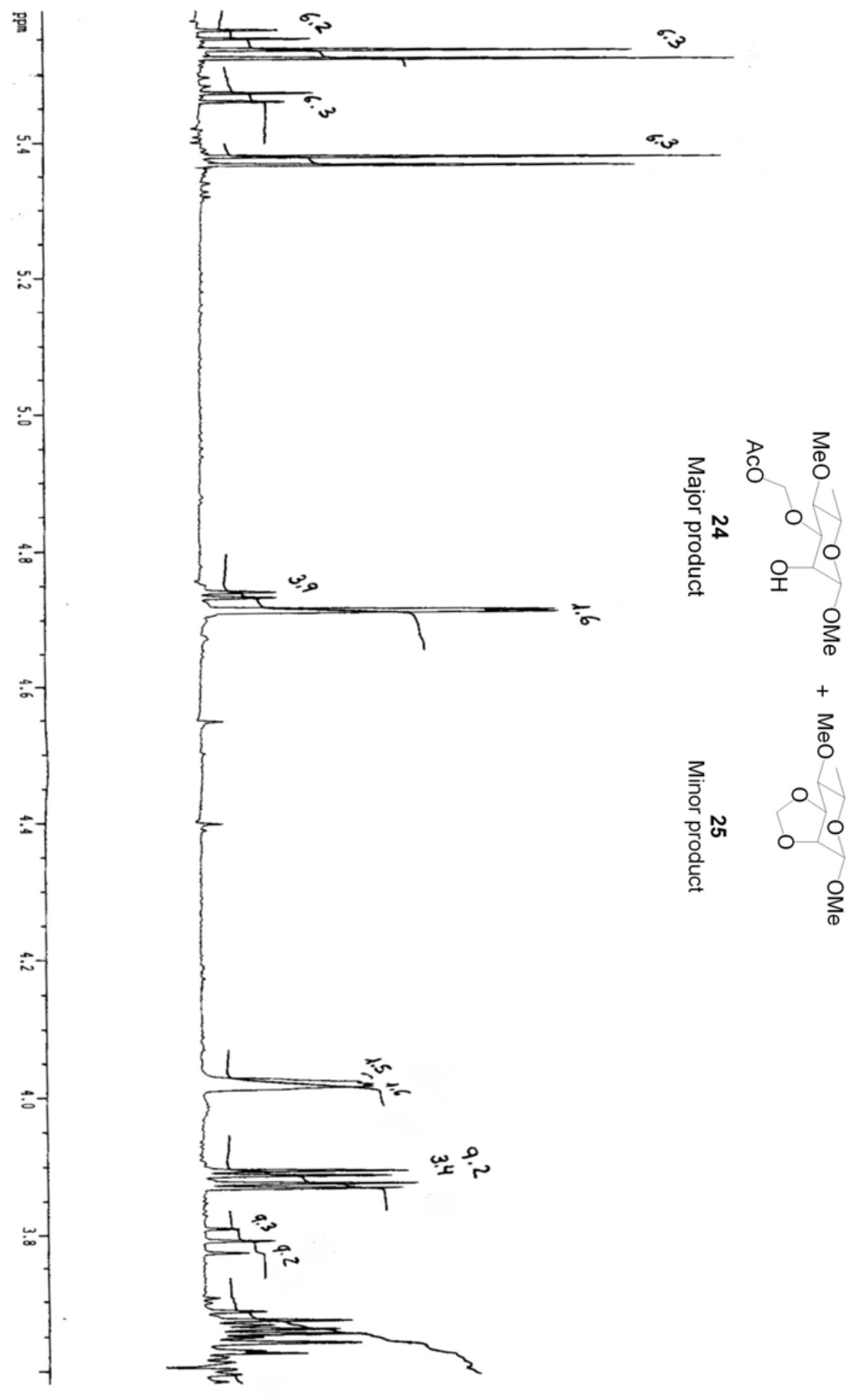




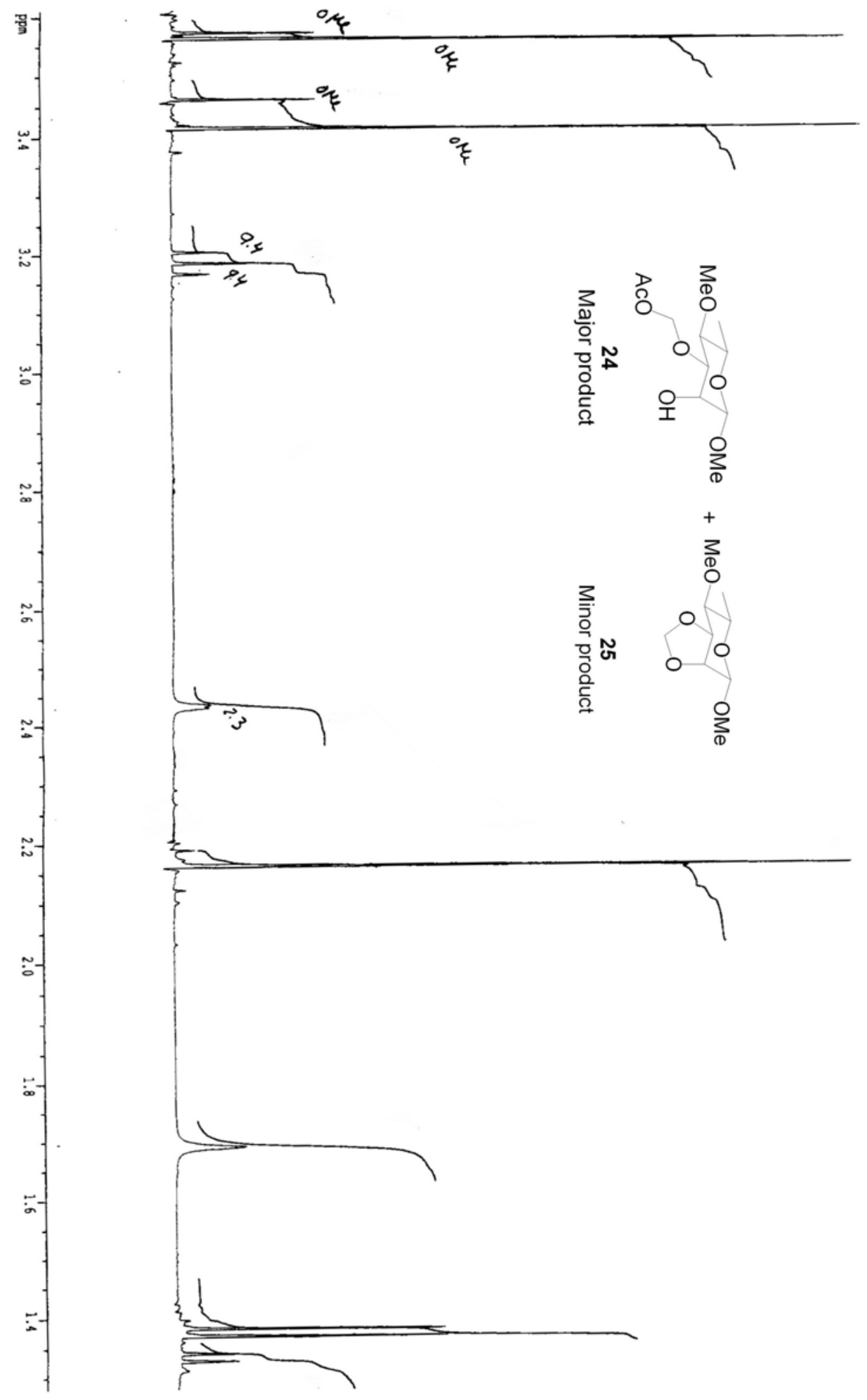




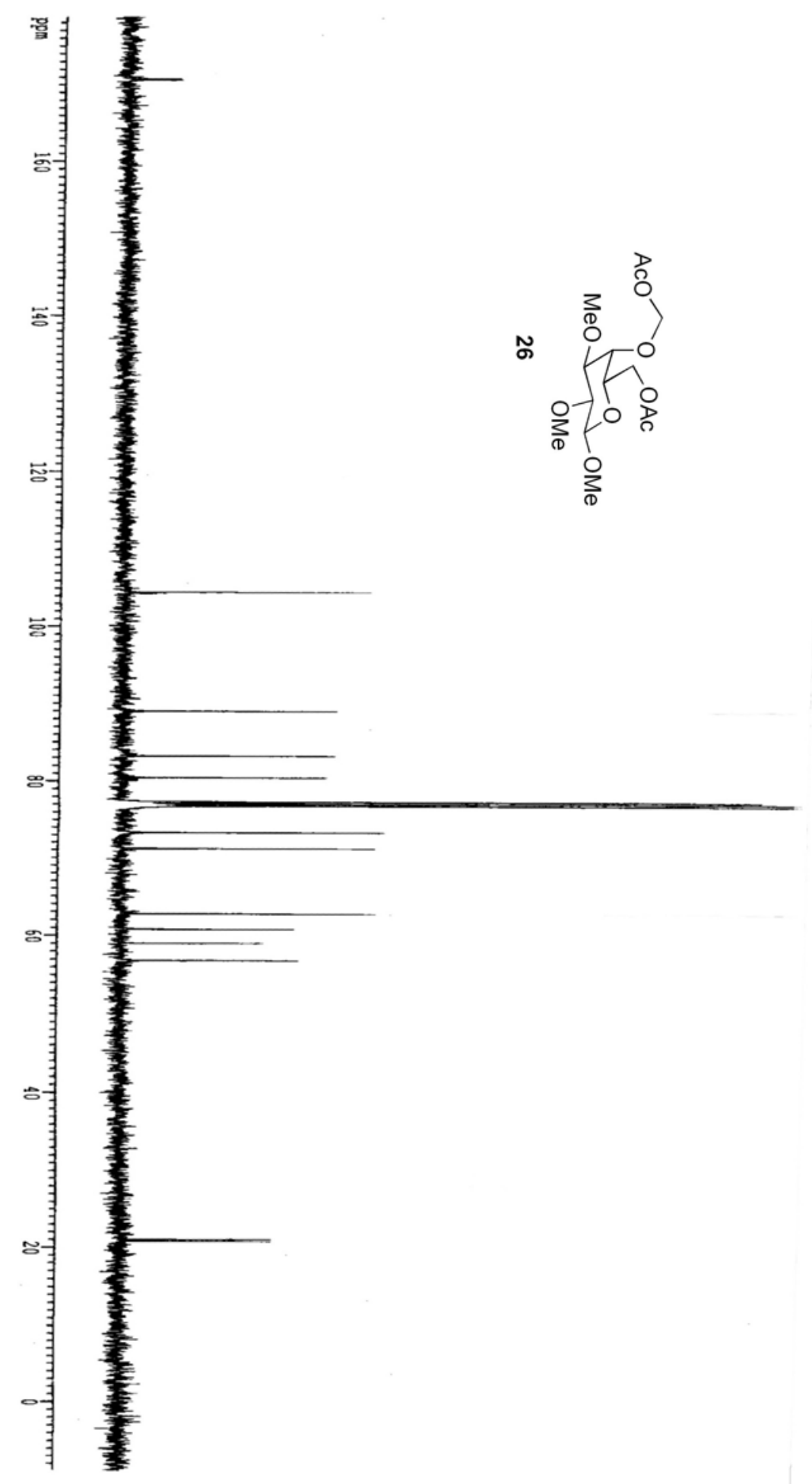




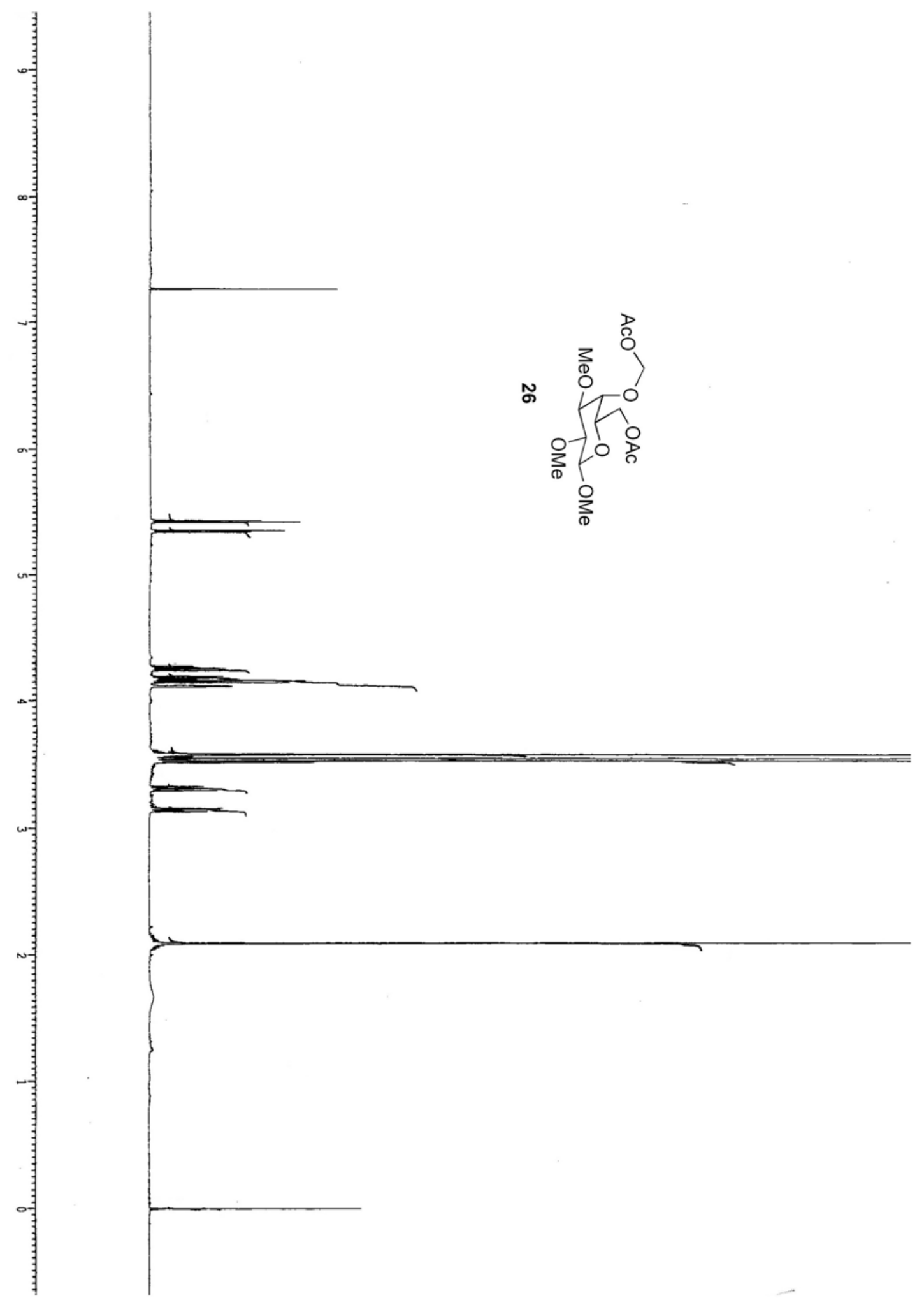




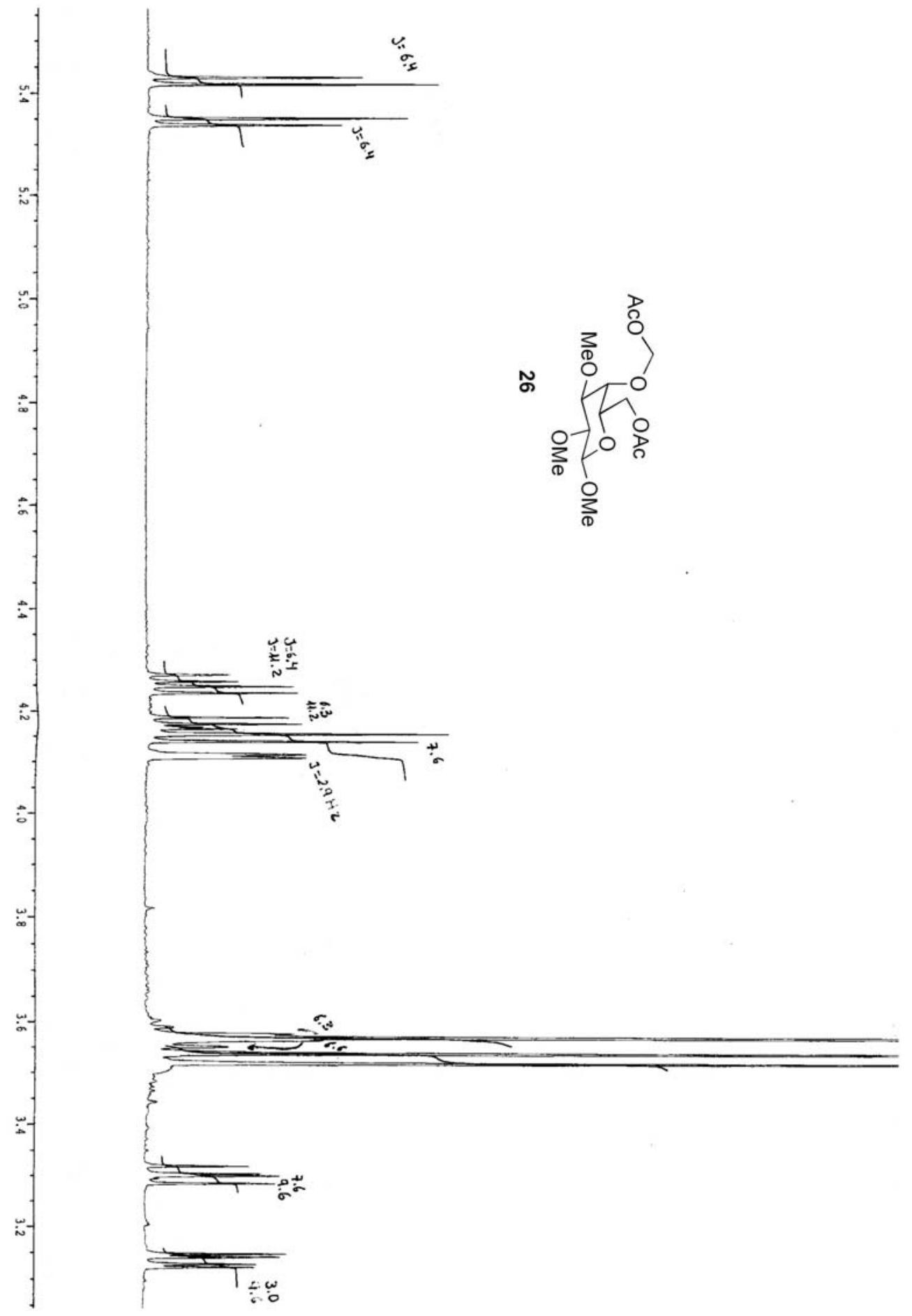




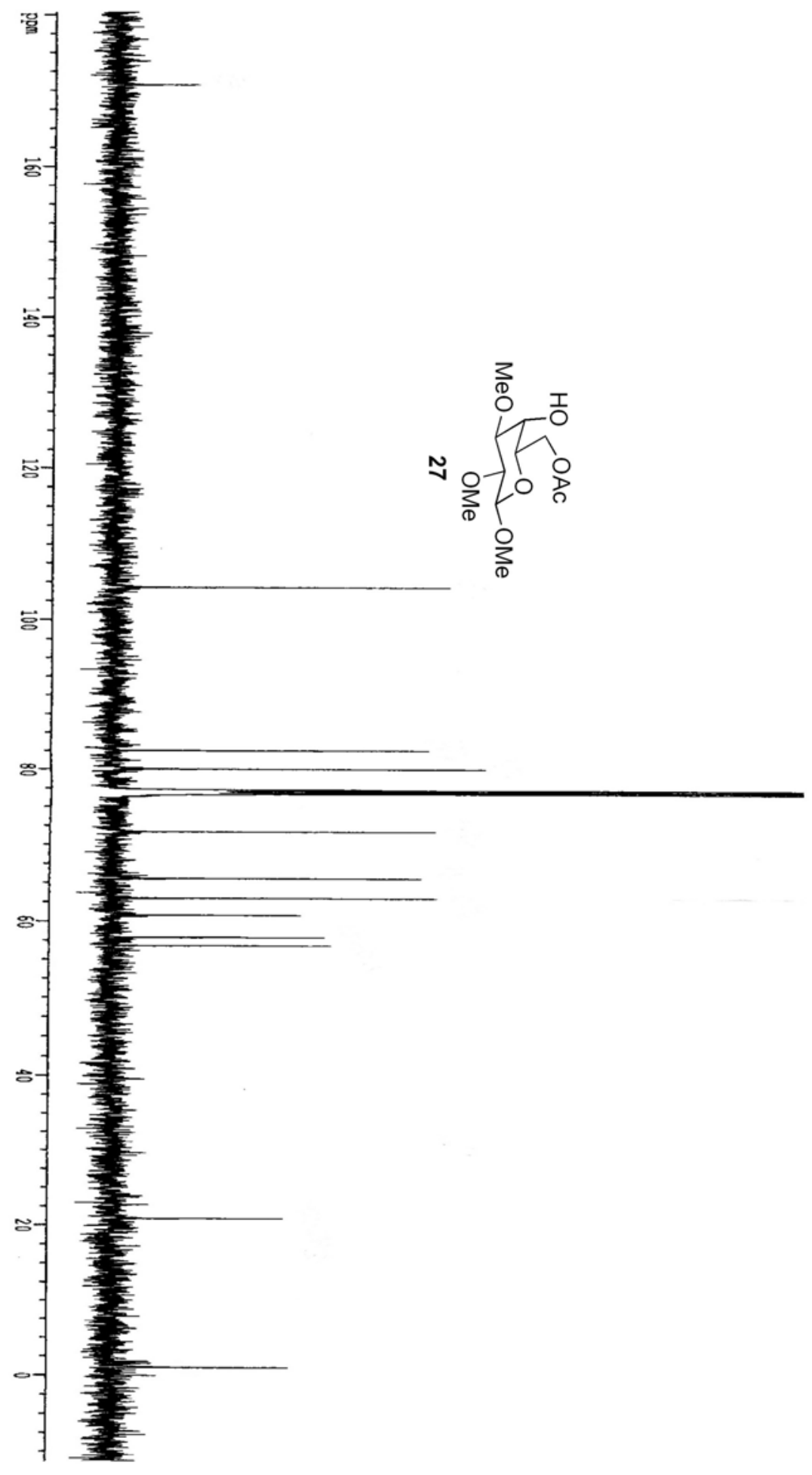




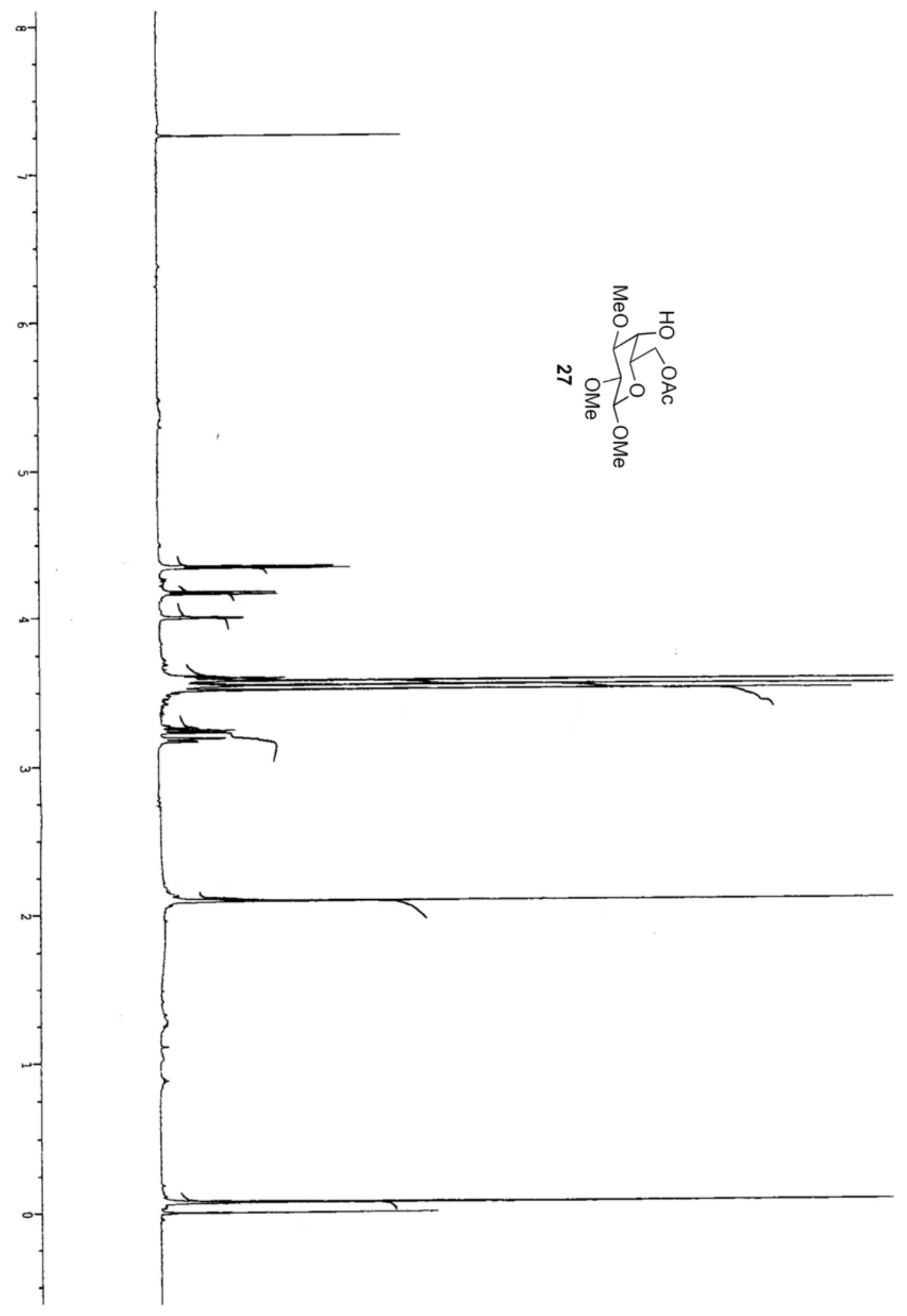




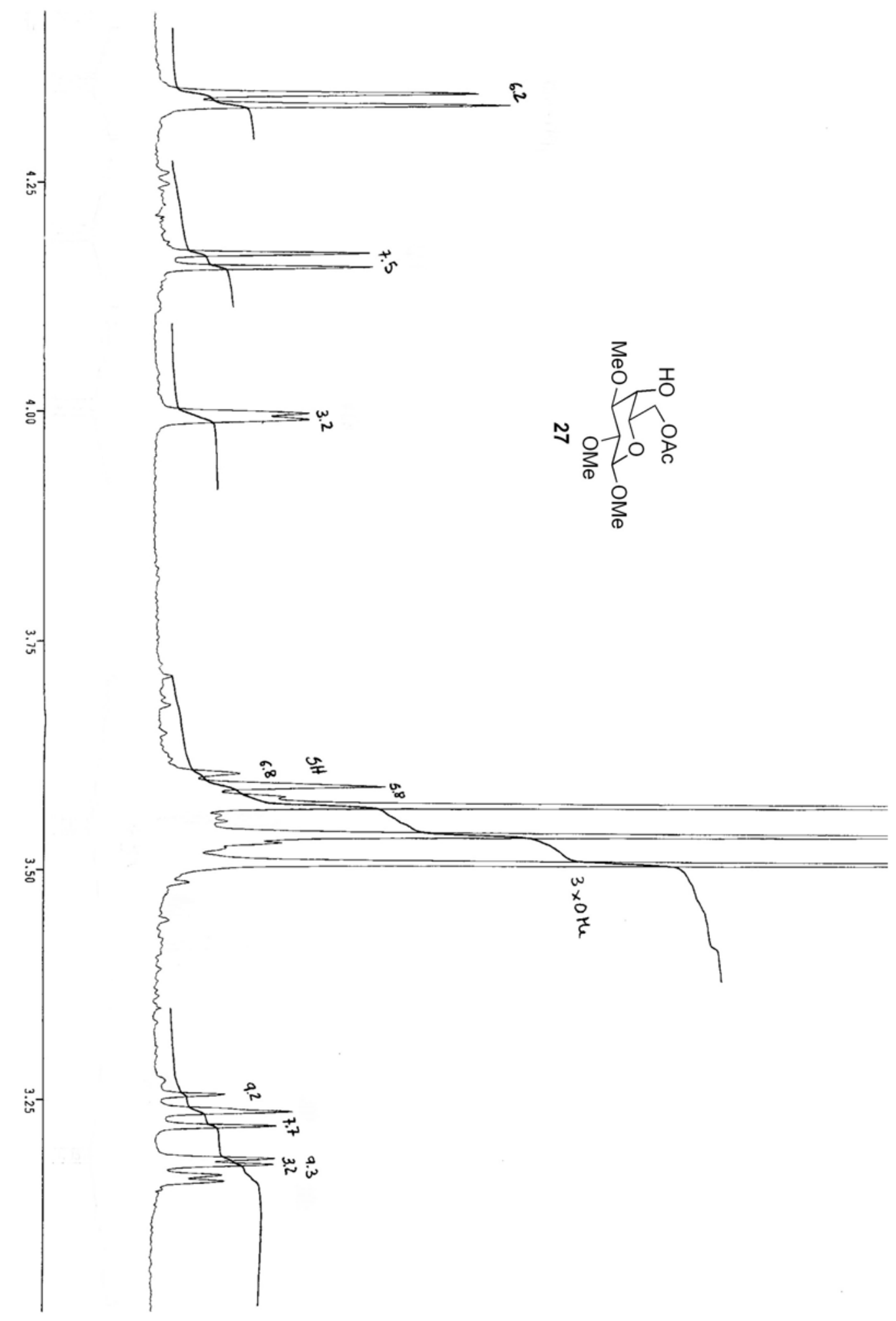




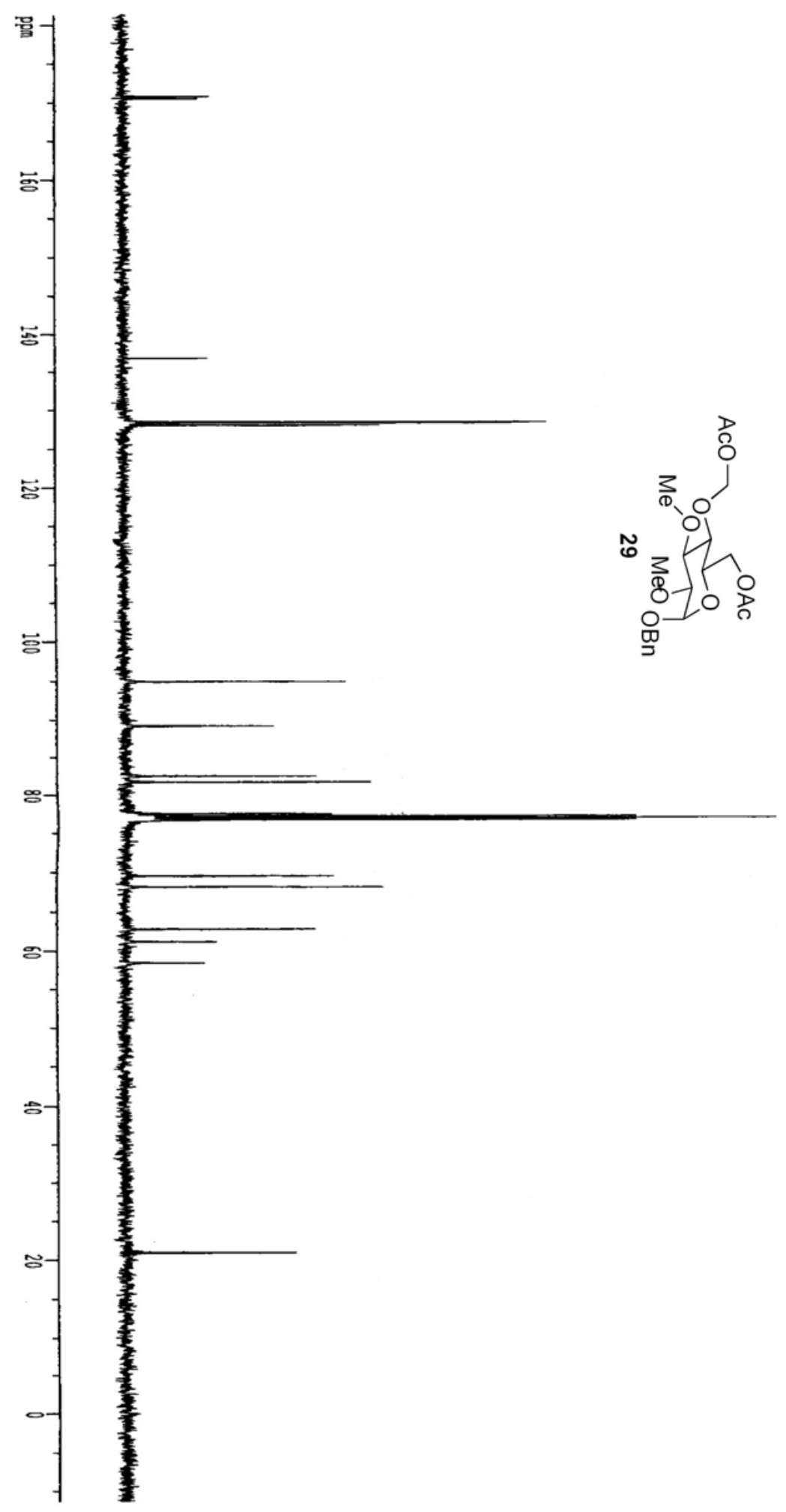




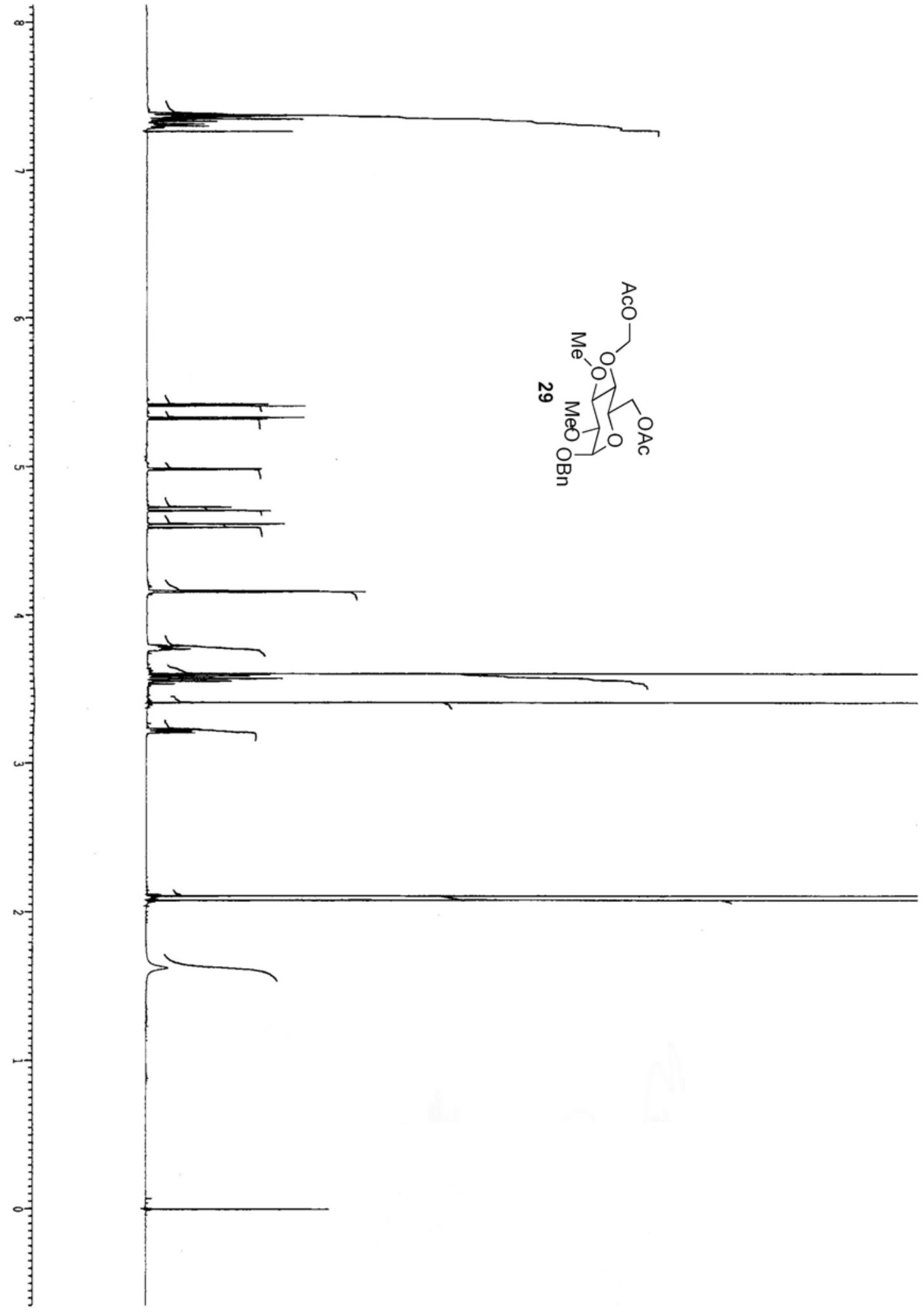




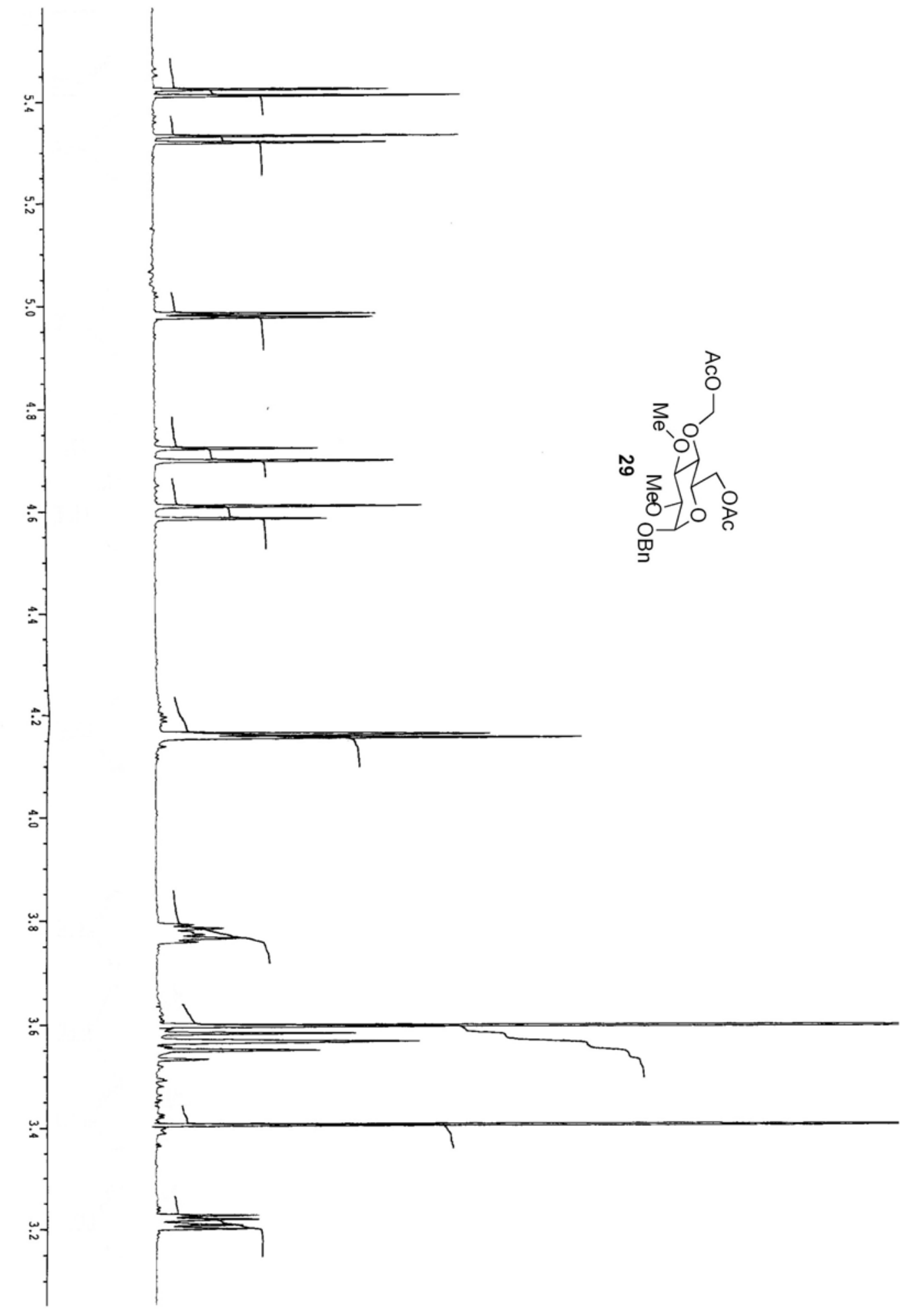




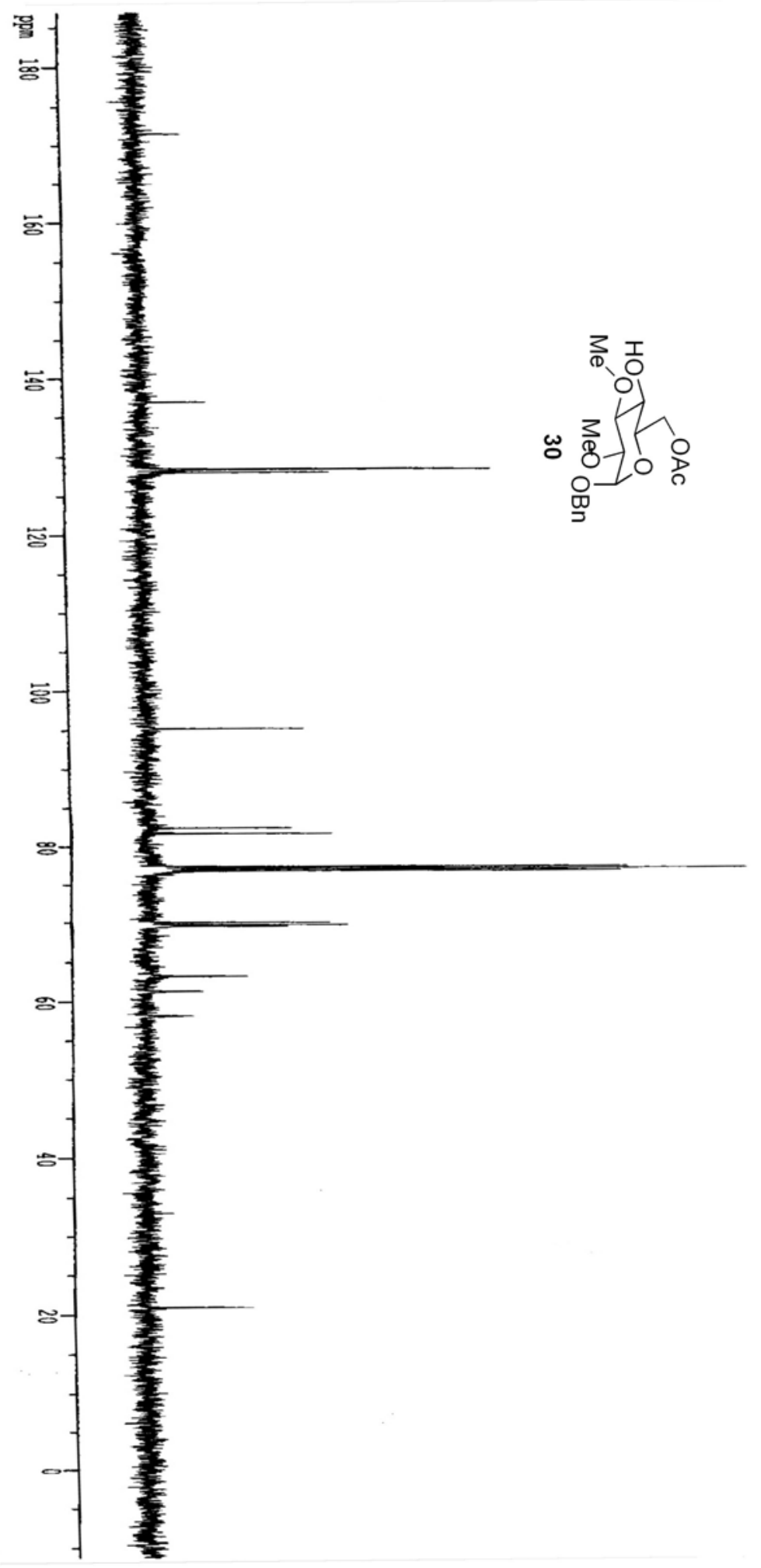




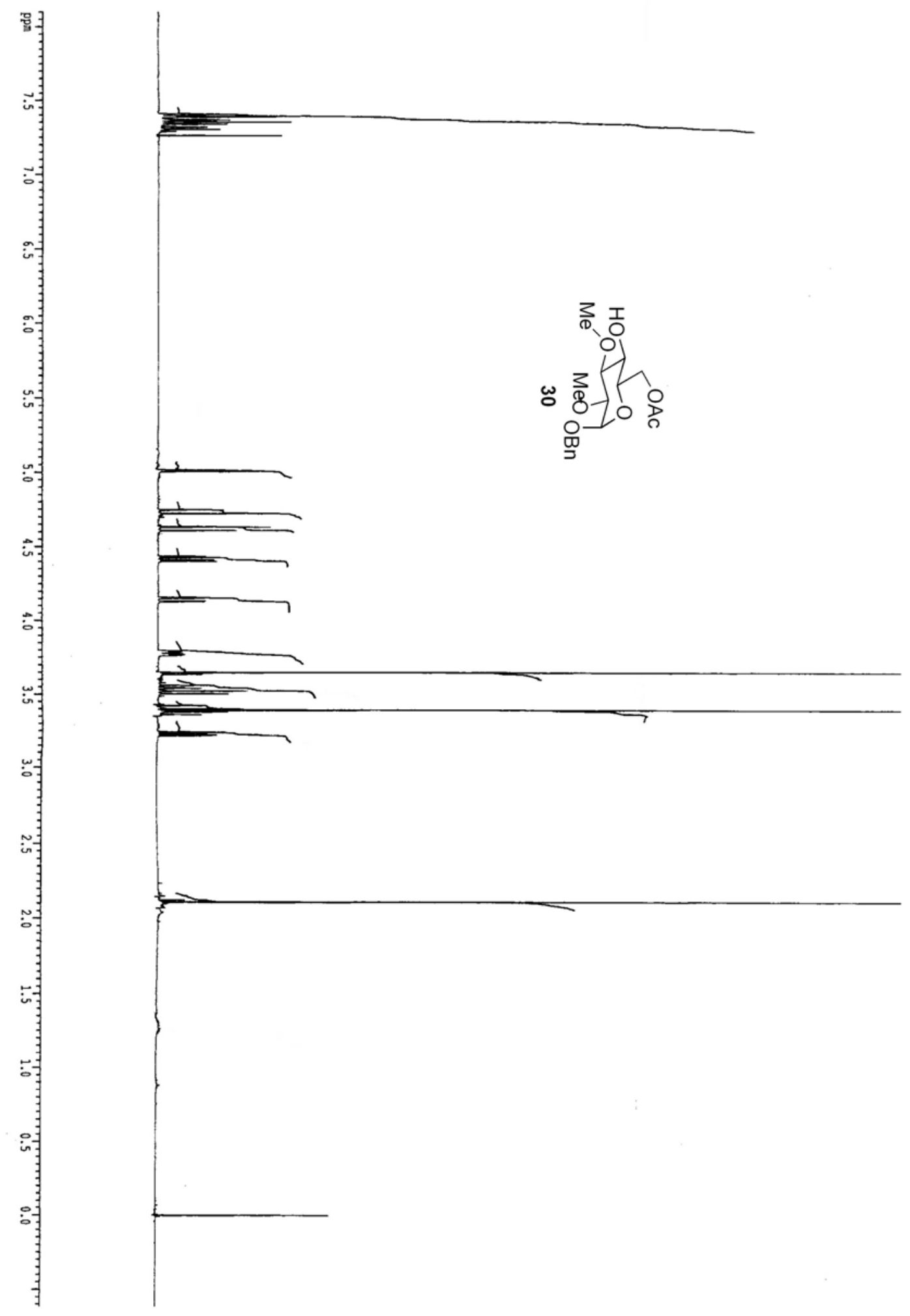




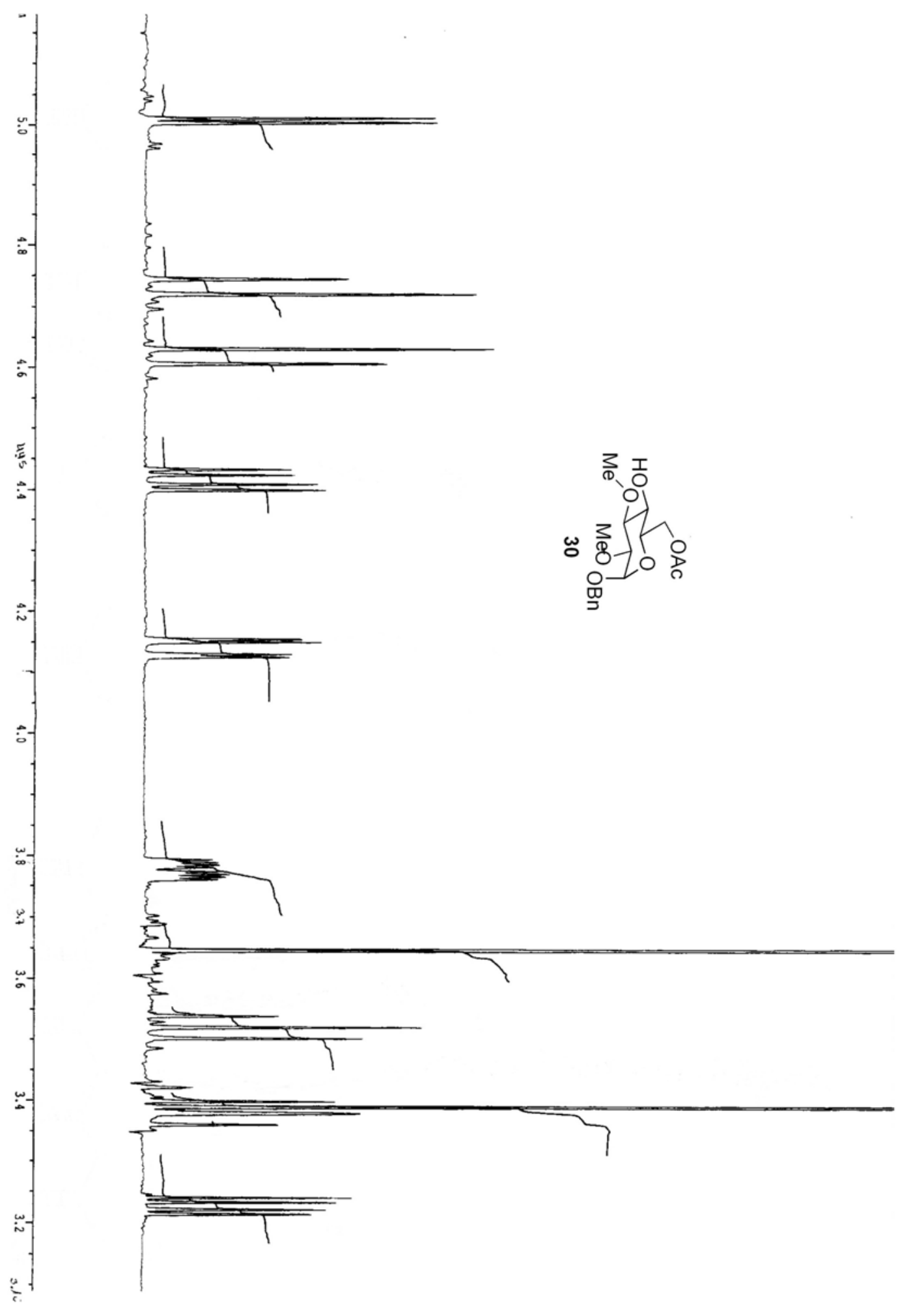




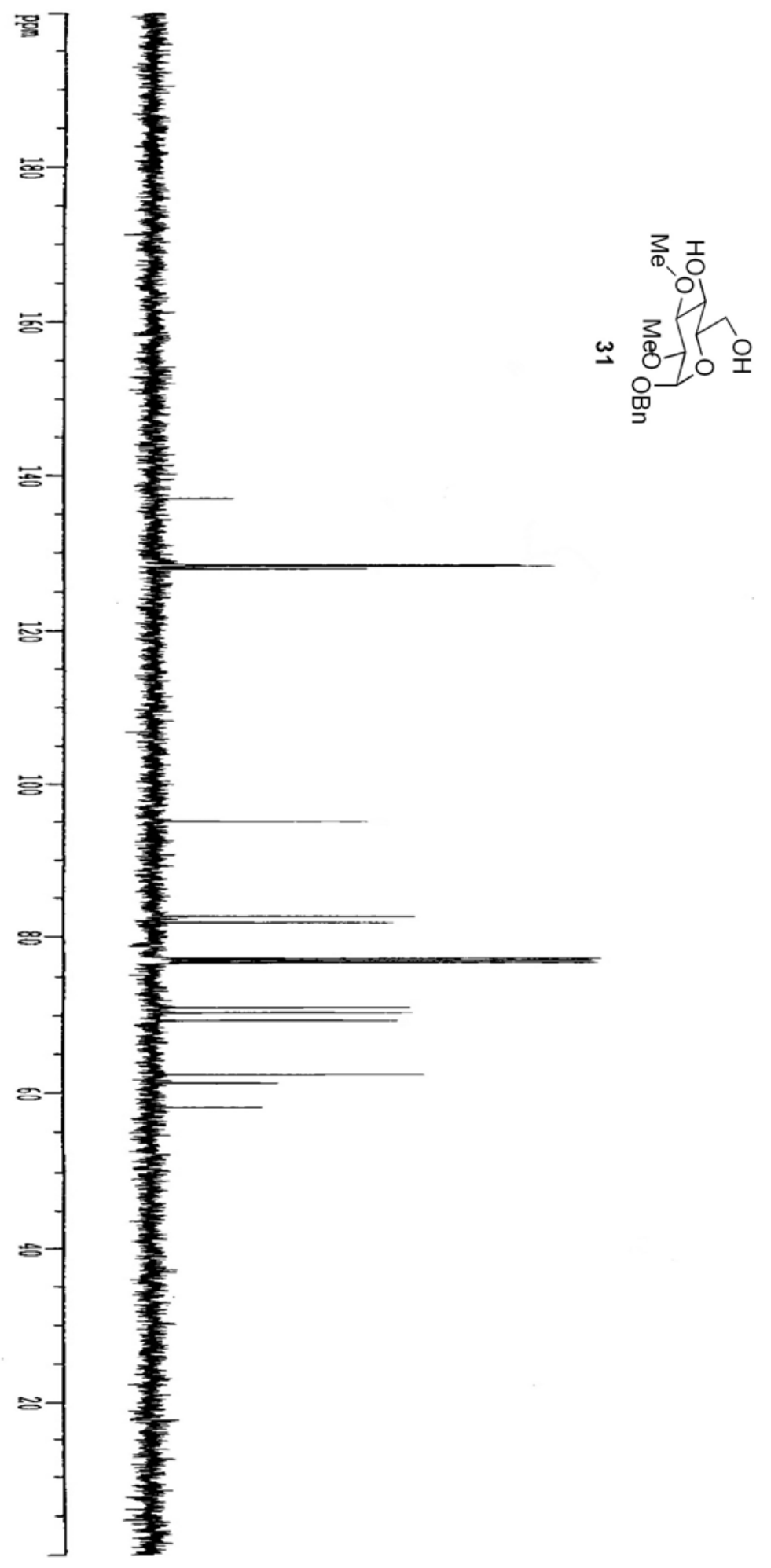




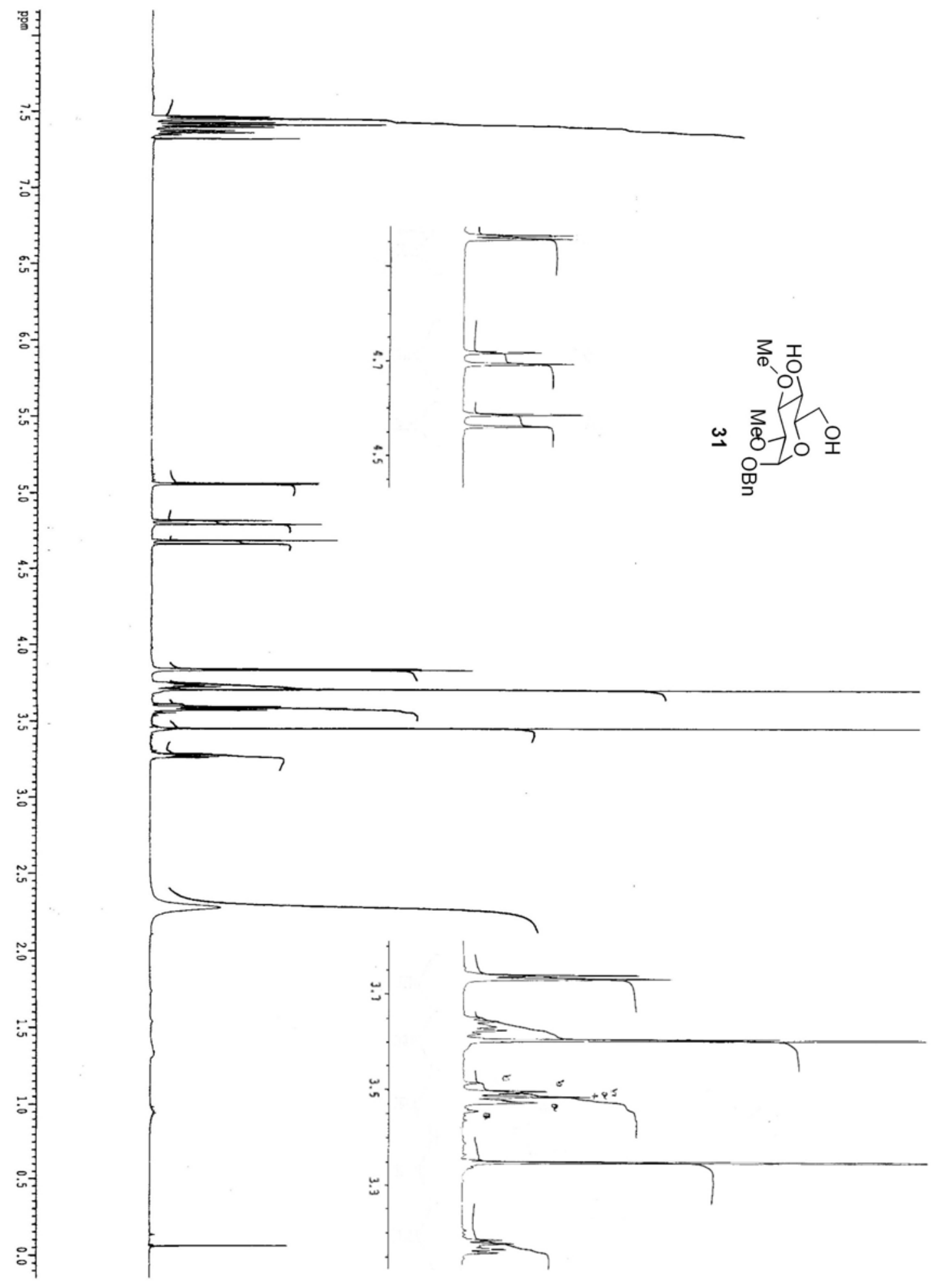




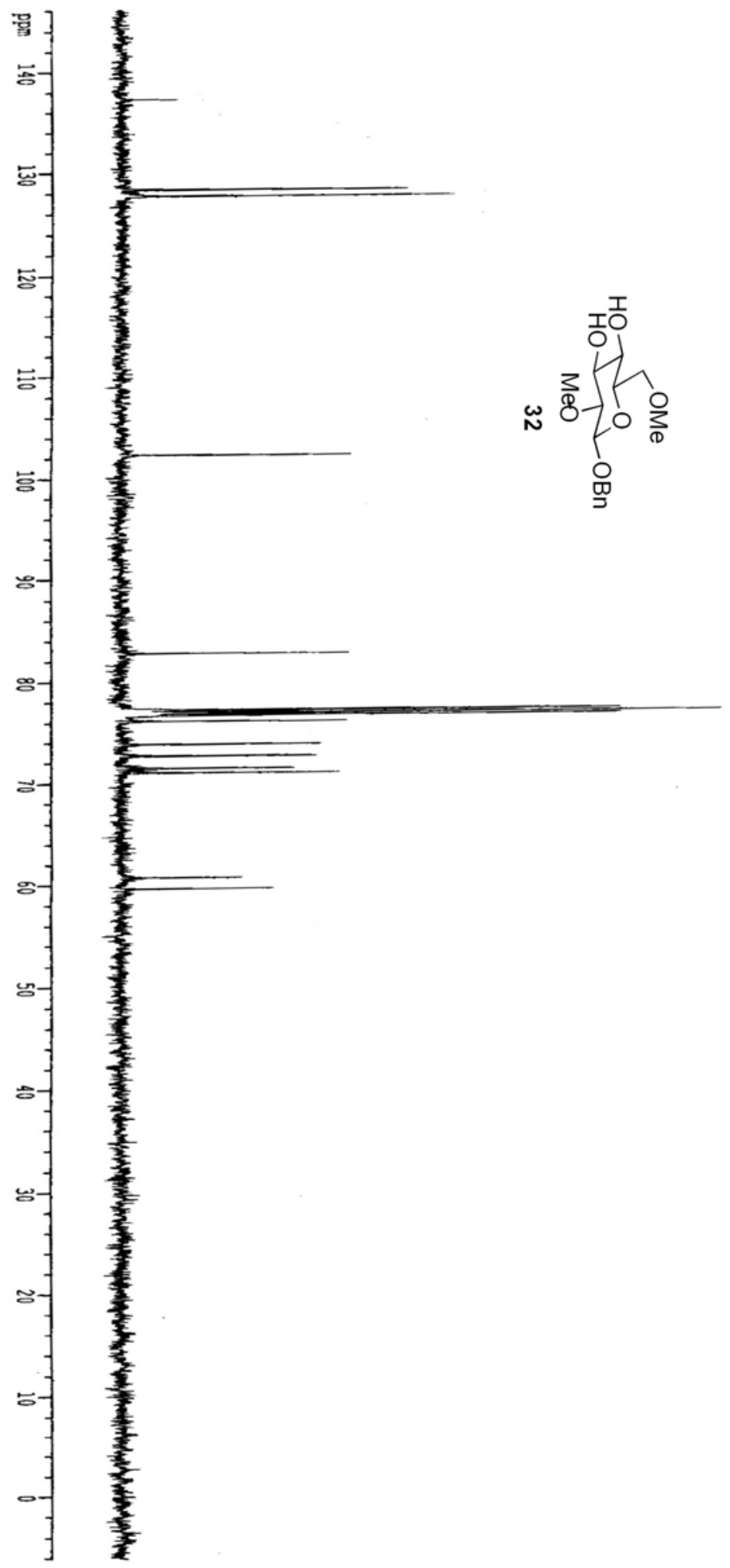




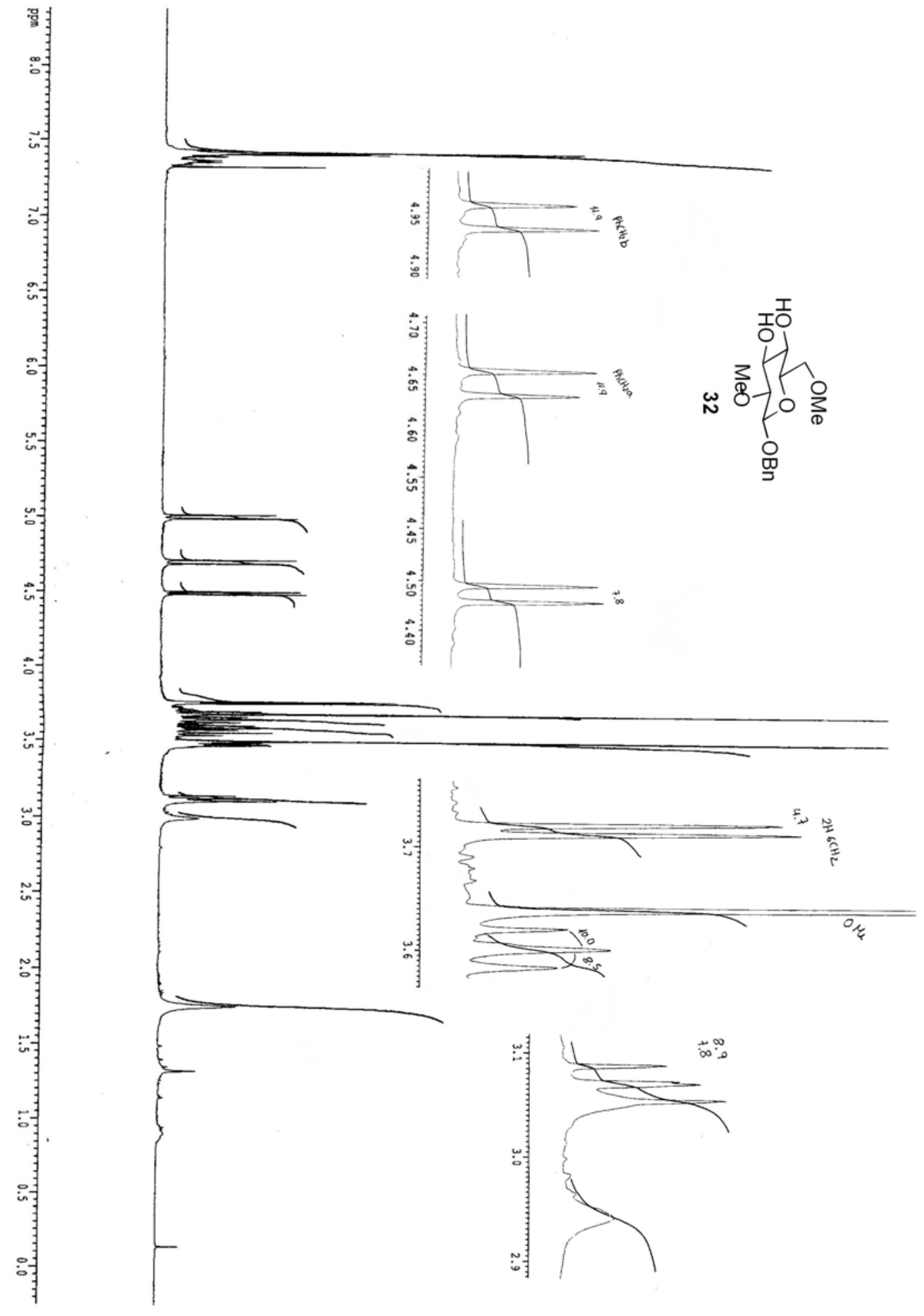

